\title{
Stochastic Calculus with respect to Gaussian processes
}

\author{
Joachim LEBOvITS*† \\ February 15, 2018
}

\begin{abstract}
Stochastic integration wrt Gaussian processes has raised strong interest in recent years, motivated in particular by its applications in Internet traffic modeling, biomedicine and finance. The aim of this work is to define and develop a White Noise Theory-based anticipative stochastic calculus with respect to all Gaussian processes that have an integral representation over a real (maybe infinite) interval. Very rich, this class of Gaussian processes contains, among many others, Volterra processes (and thus fractional Brownian motion) as well as processes the regularity of which varies along the time (such as multifractional Brownian motion). A systematic comparison of the stochastic calculus (including Itô formula) we provide here, to the ones given by Malliavin calculus in AMN01, MV05, NT06, KRT07, KR10, LN12, SV14, LN12, and by Itô stochastic calculus is also made. Not only our stochastic calculus fully generalizes and extends the ones originally proposed in [MV05] and in [NT06] for Gaussian processes, but also the ones proposed in EVdH03, BSØW04, Ben03a] for fractional Brownian motion (resp. in [LLV14, Leb13, LLVH14] for multifractional Brownian motion).
\end{abstract}

Keywords: Stochastic Analysis, White Noise Theory, Gaussian processes, Wick-Itô integrals, Itô formula, varying regularity processes.

AMS Subject Classification: 60G15; 60H40; 60H05; 60G22

\section{Introduction}

The purpose of this paper is to develop an anticipative stochastic calculus with respect to Gaussian process $G:=\left(G_{t}\right)_{t \in \mathscr{R}}$ that can be written under the form:

$$
G_{t}=\int_{\mathbf{R}} g_{t}(u) d B_{u}
$$

where $\mathbf{R}$ denotes the set of real numbers, $\mathscr{R}$ denotes a closed interval of $\mathbf{R}$ (that may be equal to $\mathbf{R}), B:=\left(B_{u}\right)_{u \in \mathbf{R}}$ is Brownian motion on $\mathbf{R}$ and $\left(g_{t}\right)_{t \in \mathscr{R}}$ is a family of a square integrable functions 1 on $\mathbf{R}$. Denote $\mathscr{G}$ the set of Gaussian processes that can be written under the form (1.1). This class of Gaussian processes contains, among many others, Volterra processes (and thus fractional Brownian motion), Gaussian Fredholm processes as well as processes the regularity of which varies along the time (such as multifractional Brownian motion). For every positive real $T$, the process $\left(V_{t}\right)_{t \in[0, T]}$ is said to be a Volterra process on $[0, T]$ (resp. a Fredholm process), if it can be written under the form:

$$
V_{t}:=\int_{0}^{t} K(t, s) d W_{s} ; \quad\left(\text { resp. } F_{t}:=\int_{0}^{T} K_{T}(t, s) d W_{s}\right), \quad \forall t \in[0, T] .
$$

where $\left(W_{s}\right)_{s \in[0, T]}$ is a Brownian motion and $K$ belongs to $L^{2}\left([0, T]^{2}, d s\right)$. Note moreover that $\mathscr{G}$ also contains the Gaussian processes that can be written under the form:

$$
H_{t}:=\int_{-\infty}^{t} K(t, s) d B_{s} ; \quad \forall t \in \mathbf{R} .
$$

Our main result is an Itô formula, that reads:

\footnotetext{
*MAThematic Center of Heidelberg, University of Heidelberg, INF 294, 69120 Heidelberg, Germany.

${ }^{\dagger}$ Laboratoire Analyse, Géométrie et Applications, C.N.R.S. (UMR 7539), Université Paris 13, Sorbonne Paris Cité, 99 avenue Jean-Baptiste Clément 93430, Villetaneuse, France. Email address: jolebovits@gmail.com

${ }^{1} i$.e. such that for all $t$ in $\mathscr{R}, u \mapsto g_{t}(u)$ is measurable on $\mathbf{R}$ and such that $\int_{\mathbf{R}}\left|g_{t}(u)\right|^{2} d u<+\infty$.
} 
- for every $T>0$ and every $C^{1,2}([0, T] \times \mathbf{R})$ function $f$, with sub exponential growth:

$$
f\left(T, G_{T}\right)=f(0,0)+\int_{0}^{T} \frac{\partial f}{\partial t}\left(t, G_{t}\right) d t+\int_{0}^{T} \frac{\partial f}{\partial x}\left(t, G_{t}\right) d^{\diamond} G_{t}+\frac{1}{2} \int_{0}^{T} \frac{\partial^{2} f}{\partial x^{2}}\left(t, G_{t}\right) d R_{t},
$$

where the equality holds in $L^{2}(\Omega)$ and almost surely, where $t \mapsto R_{t}$ denotes the variance function of $G$, which will be supposed to be a continuous function, of bounded variations; the meaning of the different terms will be explained below. The Itô formula we provide here is, at our best knowledge, one of the most general one for Gaussian processes that are not semimartingales. Itô stochastic calculus provides a non-anticipative stochastic integral wrt semimartingales. However, Itô's theory does not apply anymore when the Gaussian process considered is not a semimartingale. Twd 2 main and parallel ways have been developed over the years to build a stochastic calculus with respect to Gaussian processes; the Itô integral wrt Brownian motion being at the intersection of all these approaches. Precisely, one has the:

- trajectorial or pathwise extensions,

- functional extensions.

The trajectorial approach, initiated by [You36], provides generalizations of the Riemann-Stieltjes integral that are: the pathwise forward-type Riemann-Stieltjes integral (introduced in [Föl81]; see also [SV13] and references therein) and the pathwise generalized Lebesgue-Stieltjes integrals (introduced in [Zä98]). The reader interested in this approach, that also provides Itô formulas, will find in [FV10] a very complete overview. Let us also mention the stochastic calculus via regularization (see [RV07] and references therein), that is also a generalization of Itô integral. Since the pathwise extensions of Itô integral require, by their very definition, that the stochastic integral is built $\omega$ by $\omega$, it will clearly appear that they are of a completely different nature from our definition of stochastic integral (that will be given in Definition 2 below). For this reason we will not compare, in this work, our approach to the pathwise ones.

Our main interest here consists in the functional approach. The functional extensions are rooted in the extension of Itô integral wrt Brownian motion to anticipative integrands built by Hitsuda in [Hit72, Hit78, and Skorohod in Sko75]. In [GT82 it was proved that the stochastic integral wrt Brownian motion and the adjoint of the derivative operator, on the Wiener space, coincide. This result led to many developments in (anticipative) stochastic calculus with respect to Gaussian processes, the most significant of which is AMN01. This latter article provides, using Malliavin calculus, not only a divergence type integral with respect to continuous Volterra processes but also Itô formulas. In fact, all the functional extensions of Itô integral developed to build a stochastic integral wrt Gaussian processes so far have been developed using the divergence type integral. One can divide these functional extensions into two groups, depending if the assumptions are made on the kernel $K$ (first group) or on the covariance function $R$ (second group). The first group is composed of [AMN01] and [MV05], while the second one is composed of [NT06, KRT07, KR10, LN12, SV14]. The stochastic calculus we propose in this work belongs to the first group since the set of assumptions we make is about the kernel $g$; however it does not use the divergence type integral.

The stochastic calculus we provide here allows us to develop a White Noise Theory-based anticipative stochastic calculus with respect to all Gaussian processes that have an integral representation over a real (maybe infinite) interval. As stated in the beginning of this section, this class of Gaussian processes is very rich. Moreover, the stochastic calculus developed in the present work also allows us to get, not only Itô formulas but also Tanaka formulas, as well as occupation times formulas for local times of any $G$ in $\mathscr{G}$. While such results seem to be out

\footnotetext{
${ }^{2}$ The enlargement of filtration technique is a third method to extend Itô integral for non semimartingale (see [MY06] and references therein). However we will not discuss it in this paper since it is very rarely used in the literature.
} 
of range for most of the intrinsic methods mentioned above, they are easily obtained using the White Noise Theory-based anticipative stochastic calculus we present here (note that all the results on Gaussian local times processes obtained using the present work are presented in [Leb17]).

\section{Outline of the paper}

The remaining of this paper is organized as follows. In Section 2, we recall some basic facts about white noise theory and about the family of operators $\left(M_{H}\right)_{H \in(0,1)}$, which is instrumental for our running example, which is presented at the end of the section. In Section 3 we define the stochastic integral wrt any $G$ in $\mathscr{G}$. An Itô formula in $L^{2}(\Omega)$ is established in the first part of Section 4. A complete comparison of our Itô formula with all the Itô formulas for Gaussian processes, provided so far in the literature of functional extensions of itô integral ends this section. In Section 5, we compare our stochastic integral with respect to elements of $\mathscr{G}$, to the divergence type integrals, provided in [AMN01, MV05] and to Itô integral. In particular, we show therein how our integral fully generalizes the one built in MV05.

\section{Background on White noise theory \& on operators $\left(M_{H}\right)_{H \in(0,1)}$}

Introduced by T. Hida in Hid75, White Noise Theory is, roughly speaking, the stochastic analogous of deterministic generalized functions (also known as tempered distributions). The idea is to realize nonlinear functional on a Hilbert space as functions of white noise (which is defined as being the time derivative of Brownian motion). White Noise theory has now many application fields, such as quantum dynamics, quantum field theory, molecular biology, mathematical finance(e.g. CLV14]), among many others (see [HS04 for more details). One can find very good introductions (and more!) to White Noise Theory in HKPS93, Kuo96, Si12, (see also references therein). One may also refer to [HOUZ10] for the study, in the white noise theory's framework, of stochastic differential equations as well as stochastic partial differential equations. We recall in this section the standard set-up for classical white-noise theory. Readers interested in more details about White Noise Theory may refer to [HKPS93, Kuo96] and [Si12].

\subsection{The spaces of stochastic test functions and stochastic distributions}

Define $\mathbf{N}$ (resp. $\mathbf{N}^{*}$ ) the set of non negative integers (resp. positive integers). Let $\mathscr{S}(\mathbf{R})$ be the Schwartz space endowed with its usual topology (i.e. a family of functions $\left(f_{n}\right)_{n \in \mathbf{N}}$ of $\mathscr{S}(\mathbf{R})^{\mathbf{N}}$ is said to converge to 0 if for all $(p, q)$ in $\mathbf{N}^{2}$ we have $\left.\lim _{n \rightarrow+\infty} \sup \left\{\left|x^{p} f_{n}^{(q)}(x)\right| ; x \in \mathbf{R}\right\}=0\right)$. Denote $\mathscr{S}^{\prime}(\mathbf{R})$ the space of tempered distributions, which is the dual space of $\mathscr{S}(\mathbf{R})$, and $\widehat{F}$ or $\mathcal{F}(F)$ the Fourier transform of any element $F$ of $\mathscr{S}^{\prime}(\mathbf{R})$. For every positive real $p$, denote $L^{p}(\mathbf{R})$ the set of measurable functions $f$ such that $\int_{\mathbf{R}}|f(u)|^{p} d u<+\infty$. When $f$ belongs to $L^{1}(\mathbf{R}), \widehat{f}$ is defined on $\mathbf{R}$ by setting $\widehat{f}(\xi):=\int_{\mathbf{R}} e^{-i x \xi} f(x) d x$. Define the measurable space $(\Omega, \mathcal{F})$ by setting $\Omega:=\mathscr{S}^{\prime}(\mathbf{R})$ and $\mathcal{F}:=\mathcal{B}\left(\mathscr{S}^{\prime}(\mathbf{R})\right)$, where $\mathcal{B}$ denotes the $\sigma$-algebra of Borel sets. The BochnerMinlos theorem ensures that there exists a unique probability measure $\mu$ on $(\Omega, \mathcal{F})$ such that, for every $f$ in $\mathscr{S}(\mathbf{R})$, the map $<., f>:(\Omega, \mathcal{F}) \rightarrow \mathbf{R}$ defined by $<., f>(\omega)=<\omega, f>$ (where $\langle\omega, f\rangle$ is by definition $\omega(f)$, i.e. the action of $\omega$ on $f$ ) is a centred Gaussian random variable with variance equal to $\|f\|_{L^{2}(\mathbf{R})}^{2}$ under $\mu$. The map $f \mapsto<., f>$ being an isometry from $\left(\mathscr{S}(\mathbf{R}),<,>_{L^{2}(\mathbf{R})}\right)$ to $\left(L^{2}(\Omega, \mathcal{F}, \mu),<,>_{L^{2}(\Omega, \mathcal{F}, \mu)}\right)$, it may be extended to $L^{2}(\mathbf{R})$. One may thus consider the centred Gaussian random variable $\left\langle\right.$., $f>$, for any $f$ in $L^{2}(\mathbf{R})$. In particular, let $t$ be in $\mathbf{R}$, the indicator function $\mathbf{1}_{[0, t]}$ is defined by setting: $\mathbf{1}_{[0, t]}(s):=1$ if $0 \leq s \leq t$, $\mathbf{1}_{[0, t]}(s):=-1$ if $t \leq s \leq 0$ and $\mathbf{1}_{[0, t]}(s):=0$ otherwise. Then the process $\left(\widetilde{B}_{t}\right)_{t \in \mathbf{R}}$, where $\widetilde{B}_{t}(\omega):=\widetilde{B}(t, \omega):=\left\langle\omega, \mathbf{1}_{[0, t]}>\right.$ is a standard Brownian motion with respect to $\mu$. It then admits 
a continuous version which will be denoted $B$. Define, for $f$ in $L^{2}(\mathbf{R}), I_{1}(f)(\omega):=\langle\omega, f\rangle$. Then $I_{1}(f)(\omega)=\int_{\mathbf{R}} f(s) d B_{s}(\omega) \mu-$ a.s., where $\int_{\mathbf{R}} f(s) d B_{s}$ denotes the Wiener integral of $f$. For every $n$ in $\mathbf{N}$, let $e_{n}(x):=(-1)^{n} \pi^{-1 / 4}\left(2^{n} n !\right)^{-1 / 2} e^{x^{2} / 2} \frac{d^{n}}{d x^{n}}\left(e^{-x^{2}}\right)$ be the $n$-th Hermite function. It is well known (see Tha93]) that $\left(e_{k}\right)_{k \in \mathbf{N}}$ is a family of functions of $\mathscr{S}(\mathbf{R})$ that forms an orthonormal basis of $L^{2}(\mathbf{R}, d t)$. The following properties about the Hermite functions (the proof of which can be found in Tha93]) will be useful.

Theorem 2.1. There exist positive constants $C$ and $\gamma$ such that, for every $k$ in $\mathbf{N}$,

$$
\left|e_{k}(x)\right| \leq C\left((k+1)^{-1 / 12} \cdot \mathbf{1}_{\{|x| \leq 2 \sqrt{k+1}\}}+e^{-\gamma x^{2}} \cdot \mathbf{1}_{\{|x|>2 \sqrt{k+1}\}}\right) .
$$

Let $\left(||_{p}\right)_{p \in \mathbf{Z}}$ be the family norms defined by $|f|_{p}^{2}:=\sum_{k=0}^{+\infty}(2 k+2)^{2 p}<f, e_{k}>_{L^{2}(\mathbf{R})}^{2}$, for all $(p, f)$ in $\mathbf{Z} \times L^{2}(\mathbf{R})$. The operator $A$, defined on $\mathscr{S}(\mathbf{R})$, by setting $A:=-\frac{d^{2}}{d x^{2}}+x^{2}+1$, admits the sequence $\left(e_{n}\right)_{n \in \mathbf{N}}$ as eigenfunctions and the sequence $(2 n+2)_{n \in \mathbf{N}}$ as eigenvalues. Define, for $p$ in $\mathbf{N}$, the spaces $\mathscr{S}_{p}(\mathbf{R}):=\left\{f \in L^{2}(\mathbf{R}),|f|_{p}<+\infty\right\}$ and $\mathscr{S}_{-}(\mathbf{R})$ as being the completion of $L^{2}(\mathbf{R})$ with respect to the norm ||$_{-p}$. We summarize here the minimum background on White Noise Theory, written e.g. in [LLVH14, p. 692-693]. More precisely, let $\left(L^{2}\right)$ denote the space $L^{2}(\Omega, \mathcal{G}, \mu)$, where $\mathcal{G}$ is the $\sigma$ - field generated by $(<., f>)_{f \in L^{2}(\mathbf{R})}$. According to Wiener-Itô's theorem, for every random variable $\Phi$ in $\left(L^{2}\right)$ there exists a unique sequence $\left(f_{n}\right)_{n \in \mathbf{N}}$ of functions in $\widehat{L}^{2}\left(\mathbf{R}^{n}\right)$ such that $\Phi$ can be decomposed as $\Phi=\sum_{n=0}^{+\infty} I_{n}\left(f_{n}\right)$, where $\widehat{L}^{2}\left(\mathbf{R}^{n}\right)$ denotes the set of all symmetric functions $f$ in $L^{2}\left(\mathbf{R}^{n}\right)$ and $I_{n}(f)$ denotes the $n$-th multiple Wiener-Itô integral of $f$ with the convention that $I_{0}\left(f_{0}\right)=f_{0}$ for constants $f_{0}$. For any $\Phi:=\sum_{n=0}^{+\infty} I_{n}\left(f_{n}\right)$ satisfying the condition $\sum_{n=0}^{+\infty} n !\left|A^{\otimes n} f_{n}\right|_{0}^{2}<+\infty$, define the element $\Gamma(A)(\Phi)$ of $\left(L^{2}\right)$ by $\Gamma(A)(\Phi):=$ $\sum_{n=0}^{+\infty} I_{n}\left(A^{\otimes n} f_{n}\right)$, where $A^{\otimes n}$ denotes the $n$-th tensor power of the operator $A$ (see Jan97, Appendix E] for more details about tensor products of operators). The operator $\Gamma(A)$ is densely defined on $\left(L^{2}\right)$. It is invertible and its inverse $\Gamma(A)^{-1}$ is bounded. We note, for $\varphi$ in $\left(L^{2}\right)$, $\|\varphi\|_{0}^{2}:=\|\varphi\|_{\left(L^{2}\right)}^{2}$. For $n$ in $\mathbf{N}$, let $\operatorname{Dom}\left(\Gamma(A)^{n}\right)$ be the domain of the $n$-th iteration of $\Gamma(A)$. Define the family of norms $\left(\|\|_{p}\right)_{p \in \mathbf{Z}}$ by:

$$
\|\Phi\|_{p}:=\left\|\Gamma(A)^{p} \Phi\right\|_{0}=\left\|\Gamma(A)^{p}(\Phi)\right\|_{\left(L^{2}\right)}, \quad \forall p \in \mathbf{Z}, \quad \forall \Phi \in\left(L^{2}\right) \cap \operatorname{Dom}\left(\Gamma(A)^{p}\right) .
$$

For $p$ in $\mathbf{N}$, define $\left(\mathcal{S}_{p}\right):=\left\{\Phi \in\left(L^{2}\right): \Gamma(A)^{p}(\Phi)\right.$ exists and belongs to $\left.\left(L^{2}\right)\right\}$ and define $\left(\mathcal{S}_{-p}\right)$ as the completion of the space $\left(L^{2}\right)$ with respect to the norm \|\|$_{-p}$. As in [Kuo96], we let $(\mathcal{S})$ denote the projective limit of the sequence $\left(\left(\mathcal{S}_{p}\right)\right)_{p \in \mathbf{N}}$ and $(\mathcal{S})^{*}$ the inductive limit of the sequence $\left(\left(\mathcal{S}_{-p}\right)\right)_{p \in \mathbf{N}}$. This means in particular that $(\mathcal{S}) \subset(L)^{2} \subset(\mathcal{S})^{*}$ and that $(\mathcal{S})^{*}$ is the dual space of $(\mathcal{S})$. Moreover, $(\mathcal{S})$ is called the space of stochastic test functions while $(\mathcal{S})^{*}$ the Hida distribution space. We will note $\ll, \gg$ the duality bracket between $(\mathcal{S})^{*}$ and $(\mathcal{S})$. If $\phi, \Phi$ belong to $\left(L^{2}\right)$, then we have the equality $\ll \Phi, \varphi \gg=\langle\Phi, \varphi\rangle_{\left(L^{2}\right)}=\mathbf{E}[\Phi \varphi]$. Besides, denote $<,>$ the duality bracket between $\mathscr{S}^{\prime}(\mathbf{R})$ and $\mathscr{S}(\mathbf{R})$ and recall that every tempered distribution $F$ can be written as $F=\sum_{n=0}^{+\infty}<F, e_{n}>e_{n}$, where the convergence holds in $\mathscr{S}^{\prime}(\mathbf{R})$. The next proposition, that will be used extensively in the sequel, is a consequence of the definition of $(\mathcal{S})$ and $(\mathcal{S})^{*}$.

Proposition 2.2. Let $F$ be in in $\mathscr{S}^{\prime}(\mathbf{R})$. Define $\left\langle., F>:=\sum_{n=0}^{+\infty}<F, e_{n}><., e_{n}>\right.$. Then there exists $p_{0}$ in $\mathbf{N}$ such that that $<., F>$ belongs to $\left(\mathcal{S}_{-p_{0}}\right)$, and hence to $(\mathcal{S})^{*}$. Moreover we have $\|<., F>\|_{-p_{0}}^{2}=|F|_{-p_{0}}^{2}$. Conversely, define $\Phi:=\sum_{n=0}^{+\infty} b_{n}<., e_{n}>$, where $\left(b_{n}\right)_{n \in \mathbf{N}}$ belongs to $\mathbf{R}^{\mathbf{N}}$. Then $\Phi$ belongs to $(\mathcal{S})^{*}$ if and only if there exists an integer $p_{0}$ in $\mathbf{N}$ such that $\sum_{n=0}^{+\infty} b_{n}^{2}(2 n+2)^{-2 p_{0}}<+\infty$. In this latter case $F:=\sum_{n=0}^{+\infty} b_{n} e_{n}$ belongs to $\mathscr{S}_{-p_{0}}(\mathbf{R})$ and then to $\mathscr{S}^{\prime}(\mathbf{R})$. It moreover verifies the equality $|F|_{-p_{0}}^{2}=\sum_{n=0}^{+\infty} b_{n}^{2}(2 n+2)^{-2 p_{0}}=\|\Phi\|_{-p_{0}}^{2}$. 


\section{$2.2(\mathcal{S})^{*}$-process, $(\mathcal{S})^{*}$-derivative and $(\mathcal{S})^{*}$-integral}

Let $(\mathbf{R}, \mathcal{B}(\mathbf{R}), m)$ be a sigma-finite measure space. Through this section, $I$ denotes an element of $\mathcal{B}(\mathbf{R})$. A measurable function $\Phi: I \rightarrow(\mathcal{S})^{*}$ is called a stochastic distribution process, or an $(\mathcal{S})^{*}$-process. An $(\mathcal{S})^{*}$-process $\Phi$ is said to be differentiable at $t_{0} \in I$ if $\lim _{r \rightarrow 0} r^{-1}\left(\Phi_{t_{0}+r}-\Phi_{t_{0}}\right)$ exists in $(\mathcal{S})^{*}$. We note $\frac{d \Phi_{t_{0}}}{d t}$ the $(\mathcal{S})^{*}$ - derivative at $t_{0}$ of the stochastic distribution process $\Phi$. $\Phi$ is said to be differentiable over $I$ if it is differentiable at every $t_{0}$ of $I$. It is also possible to define an $(\mathcal{S})^{*}$-valued integral in the following way (one may refer to [Kuo96, p.245-246] or [HP57, Def. 3.7.1 p.77] for more details).

Theorem-Definition 2.1 (integral in $\left.(\mathcal{S})^{*}\right)$. Assume that $\Phi: I \rightarrow(\mathcal{S})^{*}$ is weakly in $L^{1}(I, m)$, i.e. assume that for all $\varphi$ in $(\mathcal{S})$, the mapping $u \mapsto \ll \Phi_{u}, \varphi \gg$, from $I$ to $\mathbf{R}$, belongs to $L^{1}(I, m)$. Then there exists an unique element in $(\mathcal{S})^{*}$, noted $\int_{I} \Phi_{u} m(d u)$, such that, for all $\varphi$ in $(\mathcal{S})$,

$$
\ll \int_{I} \Phi(u) m(d u), \varphi \gg=\int_{I} \ll \Phi_{u}, \varphi \gg m(d u) .
$$

We say in this case that $\Phi$ is $(\mathcal{S})^{*}$-integrable on $I$ (with respect to the measure $m$ ), in the Pettis sense. In the sequel, when we do not specify a name for the integral (resp. for the measure $m$ ) of an $(\mathcal{S})^{*}$-integrable process $\Phi$ on $I$, we always refer to the integral in Pettis' sense (resp. to the Lebesgue measure).

\subsection{S-transform and Wick product}

For $f$ in $L^{2}(\mathbf{R})$, define the Wick exponential of $\left\langle., f>\right.$, noted $: e^{<., f>}:$, as the $\left(L^{2}\right)$ random variable equal to $e^{<., f>-\frac{1}{2}|f|_{0}^{2}}$. The $S$-transform of an element $\Phi$ of $\left(\mathcal{S}^{*}\right)$, noted $S(\Phi)$, is defined as the function from $\mathscr{S}(\mathbf{R})$ to $\mathbf{R}$ given by $S(\Phi)(\eta):=\ll \Phi,: e^{<,, \eta>}: \gg$ for any $\eta$ in $\mathscr{S}(\mathbf{R})$. For any $(\Phi, \Psi) \in(\mathcal{S})^{*} \times(\mathcal{S})^{*}$, there exists a unique element of $(\mathcal{S})^{*}$, called the Wick product of $\Phi$ and $\Psi$, and noted $\Phi \diamond \Psi$, such that $S(\Phi \diamond \Psi)(\eta)=S(\Phi)(\eta) S(\Psi)(\eta)$ for every $\eta$ in $\mathscr{S}(\mathbf{R})$. Note that, when $\Phi$ belongs to $\left(L^{2}\right), S \Phi(\eta)$ is nothing but $\mathbf{E}\left[\Phi: e^{<., \eta>}:\right]=e^{-\frac{1}{2}|\eta|_{0}^{2}} \mathbf{E}\left[\Phi e^{<., \eta>}\right]$. The following result will be intensively used in the sequel.

Lemma 2.3. LLV14, Lemma 2.3.] For any $(p, q)$ in $\mathbf{N}^{2}$ and $(X, Y)$ in $\left(\mathcal{S}_{-p}\right) \times\left(\mathcal{S}_{-q}\right)$,

$$
|S(X \diamond Y)(\eta)| \leq\|X\|_{-p}\|Y\|_{-q} e^{|\eta|_{\max \{p ; q\}}^{2}} .
$$

Some useful properties of $\mathrm{S}$ transforms are listed in the proposition below. The proof of the results stated in this proposition can be found in [Kuo96, Chap 5].

Proposition 2.4 (Some properties of S transforms). When $\Phi$ is deterministic then $\Phi \diamond \Psi=\Phi \Psi$, for all $\Psi$ in $(\mathcal{S})^{*}$. Moreover, let $\Phi=\sum_{k=0}^{+\infty} a_{k}<., e_{k}>$ and $\Psi=\sum_{n=0}^{+\infty} I_{n}\left(f_{n}\right)$ be in $(\mathcal{S})^{*}$. Then their $S$-transform is given, for every $\eta$ in $\mathscr{S}(\mathbf{R})$, by $S(\Phi)(\eta)=\sum_{k=0}^{+\infty} a_{k}<\eta, e_{k}>_{L^{2}(\mathbf{R})}$ and $S(\Psi)(\eta)=\sum_{k=0}^{+\infty}<f_{n}, \eta^{\otimes n}>$. Finally, for every $(f, \eta, \xi)$ in $L^{2}(\mathbf{R}) \times \mathscr{S}(\mathbf{R}) \times \mathbf{R}$, we have the equality:

$$
S\left(e^{i \xi<., f>}\right)(\eta)=e^{\frac{1}{2}\left(|\eta|_{0}^{2}+2 i \xi<f, \eta>-\xi^{2}|f|_{0}^{2}\right)} .
$$

One may refer to [Jan97, Chap.3 and 16] for more details about Wick product. The following results on the S-transform will be used extensively in the sequel. See [Kuo96, p.39] and [HKPS93, p.280-281] for proofs. Denote $\mathcal{F}(A ; B)$ the set of $B$-valued functions defined on $A$.

Lemma 2.5. The S-transform verifies the following properties:

(i) The map $S: \Phi \mapsto S(\Phi)$, from $(\mathcal{S})^{*}$ into $\mathcal{F}(\mathscr{S}(\mathbf{R})$; $\mathbf{R})$, is injective. 
(ii) Let $\Phi: I \rightarrow(\mathcal{S})^{*}$ be an $(\mathcal{S})^{*}$ process. If $\Phi$ is $(\mathcal{S})^{*}$-integrable over $I$ wrt $m$, then one has, for all $\eta$ in $\mathscr{S}(\mathbf{R}), S\left(\int_{I} \Phi(u) m(d u)\right)(\eta)=\int_{I} S(\Phi(u))(\eta) m(d u)$.

(iii) Let $\Phi: I \rightarrow(\mathcal{S})^{*}$ be an $(\mathcal{S})^{*}$-process differentiable at $t \in I$. Then, for every $\eta$ in $\mathscr{S}(\mathbf{R})$ the map $u \mapsto[S \Phi(u)](\eta)$ is differentiable at $t$ and verifies $S\left[\frac{d \Phi}{d t}(t)\right](\eta)=\frac{d}{d t}[S[\Phi(t)](\eta)]$.

The next theorems provide a criterion for integrability in $(\mathcal{S})^{*}$, in term of $S$-transform.

Theorem 2.6. [Kuo96, Theorem 13.5] Let $\Phi: I \rightarrow(\mathcal{S})^{*}$ be a stochastic distribution such that, for all $\eta$ in $\mathscr{S}(\mathbf{R})$, the real- valued map $t \mapsto S[\Phi(t)](\eta)$ is measurable and such that there exist a natural integer $p$, a real a and a function $L$ in $L^{1}(I, m)$ such that $|S(\Phi(t))(\eta)| \leq L(t) e^{a|\eta|_{p}^{2}}$, for all $\eta$ of $\mathscr{S}(\mathbf{R})$ and for almost every $t$ of $I$. Then $\Phi$ is $(\mathcal{S})^{*}$ - integrable over $I$, wrt to $m$.

We end this section with the following theorem that will be useful in the next section.

Theorem 2.7. Ben03a, Theorem 2.17] For any differentiable map $F: I \rightarrow \mathscr{S}^{\prime}(\mathbf{R})$, the element $<, F(t)$ is a differentiable stochastic distribution process which satisfies the equality:

$$
\frac{d}{d t}<., F(t)>=<., \frac{d F}{d t}(t)>.
$$

\section{Gaussian Processes in $\mathscr{G}$ of "reference"}

To see in what extent the stochastic calculus wrt Gaussian processes we present here generalizes the one provided in the literature so far, we will consider, throughout this paper, a running example, made with elements of $\mathscr{G}$ that are Brownian motion and Brownian bridge, fractional and multifractional Brownian motions as well as $\mathscr{V}_{\gamma}$ - processes (the last three processes being defined below).

\section{Fractional and Multifractional Brownian motions}

Readers interested in an exhaustive presentation of $\mathrm{fBm}$ or $\mathrm{mBm}$ may refer to [Nua06] for $\mathrm{fBm}$ and to [LLVH14] for mBm, as well as to the references therein. Introduced in [Kol40] and popularized in [MVN68], fBm is a centered Gaussian process, the covariance function of which is denoted $R_{H}$ and is given by:

$$
R_{H}(t, s):=\frac{1}{2}\left(|t|^{2 H}+|s|^{2 H}-|t-s|^{2 H}\right),
$$

where $H$ belongs to $(0,1)$, and is usually called the Hurst exponent. When $H=1 / 2$, fBm reduces to standard Brownian motion. Among many other properties, fBm is able to match any prescribed constant local regularity and to model phenomena that presents long range dependence. These properties made this process very popular in many fields such as mathematical finance, Internet traffic modeling, image analysis and synthesis, physics and more.

$\mathrm{MBm}$, which is a Gaussian extension of fBm, was introduced in [PLV95 and in [BJR97 in order to match any prescribed non-constant deterministic local regularity and to decouple this property from long range dependence (this impossibility of doing so for fBm constitutes one of the most severe drawbacks of this process). To obtain $\mathrm{mBm}$, the idea is to replace the constant Hurst parameter $H$ of $\mathrm{fBm}$ by a deterministic function $t \mapsto h(t)$ ranging in $(0,1)$. Several definitions of $\mathrm{mBm}$ exist and the reader interested in the evolution of these definitions may refer to [LLVH14] and references therein. We will only give here the definition of $\mathrm{mBm}$ given in [LLVH14, Definition 1.2], which is not only the most recent but also includes all previously known ones. A mBm on $\mathbf{R}$, with functional parameter $h: \mathbf{R} \rightarrow(0,1)$, is a Gaussian process $B^{h}:=\left(B_{t}^{h}\right)_{t \in \mathbf{R}}$ defined, for all real $t$, by $B_{t}^{h}:=\mathbf{B}(t, h(t))$, where $\mathbf{B}:=(\mathbf{B}(t, H))_{(t, H) \in \mathbf{R} \times(0,1)}$ is fractional Brownian field on $\mathbf{R} \times(0,1)$ (which means that $\mathbf{B}$ is a Gaussian field, such that, for every $H$ in $(0,1)$, the process $(\mathbf{B}(t, H))_{t \in \mathbf{R}}$ is a $\mathrm{fBm}$ with Hurst parameter $\left.H\right)$. In other words, a $\mathrm{mBm}$ is simply a "path" traced on a fractional Brownian field. Note also that when $h$ is constant, 
$\mathrm{mBm}$ reduces to $\mathrm{fBm}$. The literature on Stochastic integration wrt $\mathrm{fBm}$ is extensive now. The reader interested in an exhaustive overview of the subject may refer to [Nua06, Cou07, Mis08] for divergence type integral and to [Ben03a, BSØW04, EVdH03, Nua05] for integral in the white noise theory framework. More recent, the literature on Stochastic integration wrt $\mathrm{mBm}$ is less rich. Nevertheless, one may cite BDM10] for a divergence type integral wrt to a Volterratype $\mathrm{mBm}$ and [LLV14, Leb13] for a Wick-Itô multifractional integral (i.e. an integral wrt to normalized $\mathrm{mBm}$, in the White Noise theory framework). Note moreover that [LLVH14] provides a general method of integration wrt to all classes of $\mathrm{mBm}$, that does not only apply for divergence type integral and white noise theory integral but also for pathwise integral.

\section{$\mathscr{V}_{\gamma}$ - processes}

MV05 provides a stochastic calculus, wrt a particular class of Volterra processes, that we will denote $\mathscr{V}_{\gamma}$ - processes in the sequel. For any deterministic function $\gamma: \mathbf{R}_{+} \rightarrow \mathbf{R}, \mathscr{V}_{\gamma}$ - processes are defined in [MV05, Proposition 1] as being the processes, denoted $\widetilde{B}^{\gamma}:=\left(\widetilde{B}_{t}^{\gamma}\right)_{t \in[0, T]}$, by setting:

$$
\widetilde{B}_{t}^{\gamma}:=\int_{0}^{t} \varepsilon(t-s) d W_{s} ; \quad \forall t \in[0, T]
$$

with $\gamma: \mathbf{R}_{+} \rightarrow \mathbf{R}$ such that $\gamma^{2}$ is of class $C^{2}$ everywhere in $\mathbf{R}_{+}$except in 0 ; and such that $\left(\gamma^{2}\right)^{\prime}$ is non increasing. The map $\varepsilon: \mathbf{R}_{+}^{*} \rightarrow \mathbf{R}$ is defined by setting $\varepsilon:=\sqrt{\left(\gamma^{2}\right)^{\prime}}$. Subset of $\mathscr{G}$, the set $\mathscr{V}_{\gamma}$ contains Gaussian processes, that can be more irregular than any $\mathrm{fBm}$. However it does not contain $\mathrm{fBm}($ nor $\mathrm{mBm})$ since $\mathscr{V}_{\gamma}$ only contains processes the regularity of which remains constant along the time). It will be shown in Remark 11 that the stochastic integral built in MV05] is a particular case of the stochastic integral we build in this work. For notational simplicity we will refer to these processes as the Gaussian processes of "reference".

\subsection{Operators $\left(M_{H}\right)_{H \in(0,1)}$ and a classical set of Gaussian processes in $\mathscr{G}$}

The operator $M_{H}$ will be useful in the sequel, not only to provide one with a representation of $\mathrm{fBm}$ and of $\mathrm{mBm}$ under the form (1.1), but also to verify that Assumptions $(\mathscr{A})$, we will make in Section 3, hold for both $\mathrm{fBm}$ and $\mathrm{mBm}$. Let $H$ belongs to $(0,1)$; following [EVdH03] and [LLV14, Section 2.2], define the $L^{2}(\mathbf{R})$-valued operator $M_{H}$, in the Fourier domain by:

$$
\widehat{M_{H}(u)}(y):=\frac{\sqrt{2 \pi}}{c_{H}}|y|^{1 / 2-H} \widehat{u}(y), \quad \forall y \in \mathbf{R}^{*},
$$

where $c_{x}$ is defined, for every $x$ in $(0,1)$ by $c_{x}:=\left(\frac{2 \pi}{\Gamma(2 x+1) \sin (\pi x)}\right)^{\frac{1}{2}}$. This operator is well defined on the homogeneous Sobolev space $L_{H}^{2}(\mathbf{R}):=\left\{u \in \mathscr{S}^{\prime}(\mathbf{R}): \widehat{u}=T_{f} ; f \in L_{l o c}^{1}(\mathbf{R})\right.$ and $\|u\|_{H}<$ $+\infty\}$, where the norm $\|\cdot\|_{H}$ derives from the inner product denoted $\langle\cdot, \cdot\rangle_{H}$, which is defined on $L_{H}^{2}(\mathbf{R})$ by:

$$
\langle u, v\rangle_{H}:=\frac{1}{c_{H}^{2}} \int_{\mathbf{R}}|\xi|^{1-2 H} \widehat{u}(\xi) \overline{\widehat{v}(\xi)} d \xi .
$$

$M_{H}$ being an isometry from $\left(L_{H}^{2}(\mathbf{R}),\|\cdot\|_{H}\right)$ into $\left(L^{2}(\mathbf{R}),\|\cdot\|_{L^{2}(\mathbf{R})}\right)$, it is clear that, for every $(H, t, s)$ in $(0,1) \times \mathbf{R}^{2},<M_{H}\left(\mathbf{1}_{[0, t]}\right), M_{H}\left(\mathbf{1}_{[0, s]}\right)>_{L^{2}(\mathbf{R})}=R_{H}(t, s)$. We will say that an $\mathrm{mBm}$ is normalized when its covariance function, denoted $R_{h}$, verifies the equality:

$$
R_{h}(t, s)=\frac{c_{h_{t, s}}^{2}}{c_{(h(t)) c_{(h(s))}}}\left[\frac{1}{2}\left(|t|^{2 h_{t, s}}+|s|^{2 h_{t, s}}-|t-s|^{2 h_{t, s}}\right)\right],
$$

where $h_{t, s}:=\frac{h(t)+h(s)}{2}$ and $c_{x}$ has been above, right after $\left.\widehat{M_{H}(u}\right)(y)$.

Example 2.8 (Gaussian Processes in $\mathscr{G}$ of "reference"). Let $H$ be real in $(0,1)$ and $h: \mathbf{R} \rightarrow$ $(0,1)$ be a deterministic measurable function. Define the processes 
$\left.B:=\left\{<., \mathbf{1}_{[0, t]}\right\rangle ; t \in \mathbf{R}\right\} ;$

$\widehat{B}:=\left\{<., \mathbf{1}_{[0, t]}-t \cdot \mathbf{1}_{[0,1]}>; t \in[0,1]\right\} ;$

$B^{H}:=\left\{<., M_{H}\left(\mathbf{1}_{[0, t]}\right)>; t \in \mathbf{R}\right\}$;

$B^{h}:=\left\{<., M_{h(t)}\left(\mathbf{1}_{[0, t]}\right)>; t \in \mathbf{R}\right\} ;$

$\widetilde{B}^{\gamma}:=\left\{<., \mathbf{1}_{[0, t)} \cdot \varepsilon(t-).\right)>$ if $\left.t \in \mathbf{R}_{+}^{*} \& \widetilde{B}_{0}^{\gamma}:=0\right\}$.

We know, thanks to Section [2.1, that $B$ is a Brownian motion on $\mathbf{R}$. Moreover, since for any $g_{t}$ in $L^{2}(\mathbf{R}),\left\langle., g_{t}>\stackrel{a . s .}{=} \int_{\mathbf{R}} g_{t}(u) d B_{u}\right.$, it is clear, in view of the definition of $\langle\cdot, \cdot\rangle_{H}$, that $B^{H}$ is a fBm of Hurst index $H$, that $B^{h}$ is a normalized $m B m$ of functional parameter $h$, that $\widehat{B}$ is Brownian bridge on $[0,1]$ and that $\widetilde{B}^{\gamma}$ is a $\mathscr{V}_{\gamma}$ - process (defined in (2.2)).

A word on notation: $B^{H}$ or $B^{h(t)}$ will always denote an fBm with Hurst index $H$ or $h(t)$, while $B^{h}$. will stand for an $\mathrm{mBm}$. Throughout this paper, unless otherwise specify, we will neither specify the value of $H$ in $(0,1)$, when we consider a $\mathrm{fBm}$, nor the $(0,1)$-valued function $h$ when we consider a $\mathrm{mBm}$, nor the function $\gamma$ of a $\mathscr{V}_{\gamma}$ - process.

\section{Stochastic integral with respect to Gaussian process}

The first part of this section is devoted to the definition of the time derivative, in the Stochastic distribution sense, of any element $G:=\left(G_{t}\right)_{t \in \mathscr{R}}$ of $\mathscr{G}$. We then compute the $S$-transforms of processes $G$ and of its time derivative. The Wiener integral wrt $G$ is presented in Subsection 3.4, whereas the stochastic integral wrt $G$ is built in Subsection 3.5. We keep the same notations as in Section 2. In particular, the probability space $(\Omega, \mathcal{F}, \mu)$, described in the previous section is now fixed. Denote $G:=\left(G_{t}\right)_{t \in \mathscr{R}}$ the process defined, for every $t$ in $\mathscr{R}$, by $G_{t}:=<., g_{t}>$, where $\left(g_{t}\right)_{t \in \mathscr{R}}$ is a family of functions of $L^{2}(\mathbf{R})$. As we saw in Example 2.8, $G$ is a Gaussian process which fulfills the equality $G_{t} \stackrel{\text { a.s. }}{=} \int_{\mathbf{R}} g_{t}(u) d B_{u}$. Denote $(t, s) \mapsto R_{t, s}$ the covariance function of $G$. We hence have $R_{t, s}:=\mathbf{E}\left[G_{t} G_{s}\right]=<g_{t}, g_{s}>_{L^{2}(\mathbf{R})}$, for every $(s, t)$ in $\mathscr{R}^{2}$. We will note in the sequel $R_{t}$ instead of $R_{t, t}$. For the sake of notational simplicity we assume that $G_{0} \stackrel{a . s .}{=} 0$. Moreover, when the Gaussian process $G$ will admit a continuous modification, we will systematically use it and still call it $G$.

\subsection{White Noise derivative of $G$}

Define the map $g: \mathscr{R} \rightarrow \mathscr{S}^{\prime}(\mathbf{R})$ by setting $g(t):=g_{t}$. When $g$ is differentiable at point $t$, one denotes $g_{t}^{\prime}$ its derivative. Denote $\lambda$ the Lebesgue measure on $\mathscr{R}$ and define $L_{l \widetilde{c}}^{1}(\mathscr{R}):=\{f: \mathscr{R} \rightarrow$ $\mathbf{R}$ is measurable ; $f \in L^{1}((a, b))$, for all finite interval $(a, b)$ s.t. $\left.[a, b] \subset \mathscr{R}\right\}$. In this section and in the next one (namely in Sections 3 and 4 ), we will make the following assumption:

$(\mathscr{A}) \quad\{$ (iii)

(i) The map $g$ is continuous on $\mathscr{R}$,

(ii) The map $g$ is differentiable $\lambda$-almost everywhere on $\mathscr{R}$,

(iii) There exists $q$ in $\mathbf{N}^{*}$ such that $t \mapsto\left|g_{t}^{\prime}\right|_{-q}$ belongs to $L_{l \widetilde{o c}}^{1}(\mathscr{R})$,

(iv) For every $(a, b)$ in $\mathscr{R}^{2}$ such that $a \leq b$, one has, in $\mathscr{S}^{\prime}(\mathbf{R})$, the equality:

$$
g_{b}-g_{a}=\int_{a}^{b} g_{u}^{\prime} d u
$$

Proposition 3.1 below will provide an easy way to check whether Assumption $(\mathscr{A})$ holds or not. Besides, define the set $\mathscr{R}_{D}$ by setting $\mathscr{R}_{D}:=\{t \in \mathscr{R} ; g$ is differentiable at point $t\}$. Of course $L_{l \widetilde{o c}}^{1}(\mathscr{R})$ contains in particular all measurable functions $f: \mathscr{R}_{D} \rightarrow \mathbf{R}$ such that $f \in$ $L^{1}\left((a, b) \cap \mathscr{R}_{D}\right)$ (that we will denote $L^{1}((a, b))$ in the sequel, by abuse of notation), for every 
finite interval $(a, b)$ s.t. $[a, b] \subset \mathscr{R}$. For the sake of notational simplicity we will write $\mathscr{A}_{(\mathrm{i})}$,

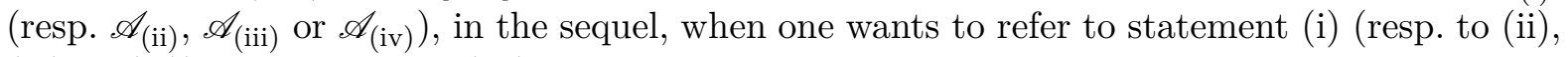
(iii) or (iv)) of Assumption ( $\mathscr{A})$.

Making Assumptions $\mathscr{A}_{(\mathrm{i})}$ and $\mathscr{A}_{(\mathrm{ii})}$ seems reasonable since we want to "differentiate", with respect to $t$, the Gaussian process $G$, which trajectories are, in general, not differentiable in the strong sense (e.g. the Brownian motion). The interest of Assumptions $\mathscr{A}_{\text {(iii) }}$ and $\mathscr{A}_{\text {(iv) }}$ will be explained when it will be needed (in Section 3.4, right after Definition 11).

Remark 1. 1. A first consequence of Assumption $(\mathscr{A})$ is that $g$ is "weakly" locally absolutely continuous on $\mathscr{R}$; that is that the map $t \mapsto<g_{t}, \eta>$ is absolutely continuous on every finite interval $[a, b]$ of $\mathscr{R}$, for every $\eta$ in $\mathscr{S}(\mathbf{R})$.

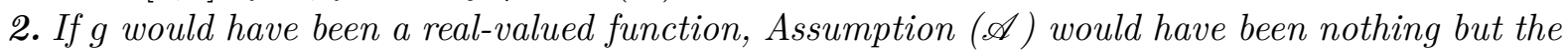
local absolute continuity of $g$ on $\mathscr{R}$. However, $g$ is $\mathscr{S}^{\prime}(\mathbf{R})$-valued. Thus, and even if a notion of absolute continuity exists for $\mathscr{S}^{\prime}(\mathbf{R})$-valued functions (see [HP57, Definitions 3.6.2 83 3.2.4]), the absolute continuity of $g$ on an interval $[a, b]$ of $\mathscr{R}$ does not entail the differentiability of $g$ in general (see an example that illustrates this fact in [HP57], right above Theorem 3.8.6).

An easy way to see if Assumption $(\mathscr{A})$ holds is to check if the sufficient condition provided in the following proposition, and that will be used a lot in the sequel, holds.

Proposition 3.1. A sufficient condition for Assumption $(\mathscr{A})$ to be verified is that:

$\{$ (i) The map $g$ is continuous on $\mathscr{R}$ and differentiable on every finite interval $(a, b)$ s.t. $[a, b] \subset \mathscr{R}$, ( (ii) There exists $q$ in $\mathbf{N}^{*}$ such that $t \mapsto\left|g_{t}^{\prime}\right|_{-q}$ belongs to $L_{l \widetilde{c c}}^{1}(\mathscr{R})$.

Proof. Indeed, these two conditions obviously entail that Assumptions $\mathscr{A}_{\text {(i) }}$ to $\mathscr{A}_{\text {(iii) }}$ hold. Moreover, these two conditions also entail Equality $\left(E_{c, d}\right)$, for every $[c, d]$ in $\mathscr{R}_{D}$. $\mathscr{R}$ being a closed interval of $\mathbf{R}$, and in view of $(i i)$ of Assumption $(\mathscr{D})$, there can be 0,1 or 2 points, at the maximum, that belong to $\mathscr{R}$ but not to $\mathscr{R}_{D}$. Let us treat this latter case only and denote $a$ and $b$ these two points. The continuity of $g$ at points $a$ and $b$, from one hand, and the Lebesgue dominated convergence theorem, from the other hand, give us the equality $\left(E_{a, b}\right)$.

As previously, and for the sake of notational simplicity we will write $\mathscr{D}_{(\mathrm{i})}\left(\right.$ resp. $\left.\mathscr{D}_{(\mathrm{ii})}\right)$ when

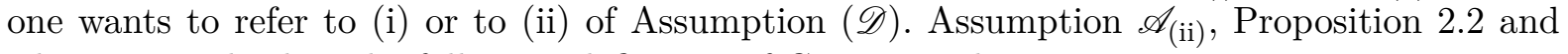
Theorem 2.7 lead to the following definition of Gaussian white noise.

Theorem-Definition 3.1 (Gaussian White Noise). Define for every $t$ in $\mathscr{R}_{D}$,

$$
W_{t}^{(G)}:=<., g_{t}^{\prime}>
$$

where the equality holds in $(\mathcal{S})^{*}$. Then $\left(W_{t}^{(G)}\right)_{t \in \mathscr{R}_{D}}$ is a $(\mathcal{S})^{*}$-process and is the $(\mathcal{S})^{*}$-derivative of the process $\left(G_{t}\right)_{t \in \mathscr{R}_{D}}$. We will sometimes note $\frac{d G_{t}}{d t}$ instead of $W_{t}^{(G)}$.

Using Proposition 2.2 one easily sees that (3.3) also reads, for every $t$ in $\mathscr{R}_{D}$ :

$$
W_{t}^{(G)}=\sum_{k=0}^{+\infty}<g_{t}^{\prime}, e_{k}><., e_{k}>=\sum_{k=0}^{+\infty}\left(\frac{d}{d t}<g_{t}, e_{k}>\right)<., e_{k}>.
$$

Proposition 3.2. The map $t \mapsto\left\|W_{t}^{(G)}\right\|_{-p}$ is continuous if and only if $t \mapsto\left|g_{t}^{\prime}\right|_{-p}$ is continuous.

Proof. Thanks to Proposition 2.2, one can write $\left\|W_{t}^{(G)}\right\|_{-p}=\left|g_{t}^{\prime}\right|_{-p}, \forall(p, t)$ in $\mathbf{N}^{*} \times \mathscr{R}_{D}$.

As the next example shows Assumptions $(\mathscr{D})$ (and therefore Assumption $(\mathscr{A})$ holds in the case of all Gaussian processes in $\mathscr{G}$ of "reference". Denote, for every $n$ in $(1 / 2,+\infty)$,

$$
\mathcal{R}_{n}:=\sum_{k=0}^{+\infty}(2 k+2)^{-2 n}
$$


Example 3.3. 1. (Brownian motion on $\mathbf{R} \&$ Brownian bridge on $[0,1]$ ). For the Brownian motion on $\mathbf{R}$ (resp. the Brownian bridge on $[0,1]$ ), one has $\mathscr{R}=\mathscr{R}_{D}=\mathbf{R}$, and, for every real $t, g_{t}^{\prime}=\delta_{t}$ (resp. $\mathscr{R}=\mathscr{R}_{D}=[0,1]$ and $\left.g_{t}^{\prime}=\delta_{t}-\mathbf{1}_{[0,1]}\right)$. Both maps $g$ clearly fulfills Asumption $\mathscr{D}_{(i)}$. Moreover, for every $p$ in $\mathbf{N}^{*}$, the maps $t \mapsto\left|g_{t}^{\prime}\right|_{-p}$ are continuous and bounded on $\mathbf{R}$, which shows that $\mathscr{D}_{(i i)}$ holds. Indeed, using both: the relation $e_{k}^{\prime}(x)=\sqrt{\frac{k}{2}} e_{k-1}(x)-\sqrt{\frac{k+1}{2}} e_{k+1}(x)$, valid for all positive integer $k$, and Theorem 2.1, we get the existence of a real $C^{\prime}$, independent of $t$ and $p$, such that: $\forall(p, t) \in \mathbf{N}^{*} \times \mathbf{R}, \quad\left|\delta_{t}^{\prime}\right|_{-p}^{2}=\sum_{k=0}^{+\infty} e_{k}^{2}(t)(2 k+2)^{-2 p} \leq C^{\prime} \cdot \mathcal{R}_{p}$.

2. (Fractional 85 Multifractional Brownian motions on $\mathbf{R}$ ) In both these cases, one has $\mathscr{R}=$ $\mathscr{R}_{D}=\mathbf{R}$. Thanks to [LLV14, Remark 4.3 and Proposition 4.10], we know that Asumption $\mathscr{D}_{(i)}$, as well as the fact that $t \mapsto\left\|W_{t}^{\left(B^{H}\right)}\right\|_{-p}$ (resp. $t \mapsto\left\|W_{t}^{\left(B^{h}\right)}\right\|_{-p}$ ) is continuous and bounded on any compact set of $\mathbf{R}$, are verified for every $p \geq 2$ and $H$ in $(0,1)$ (resp. $h$ differentiable with locally bounded derivative).

3. (The process $\widetilde{B}^{\gamma}$ ) In this case, $\mathscr{R}=\mathbf{R}_{+}$and $\mathscr{R}_{D}=\mathbf{R}_{+}^{*}:=\mathbf{R} \backslash\{0\}$. Moreover Assumption $(\mathscr{D})$ is also fulfiled, as Theorem 3.4 below shows.

The following theorem, the proof of which can be found in Appendix B.1, shows that $\mathscr{V}_{\gamma}$ - pro-

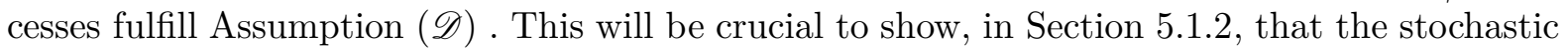
integral wrt $\mathscr{V}_{\gamma^{-}}$processes, developed in [MV05, is a particular case of the one we build in this work. Let us first define the two maps $E: \mathbf{R}_{+} \rightarrow \mathbf{R}$ and $\mathscr{E}: \mathbf{R}_{+} \rightarrow \mathbf{R}$ by setting:

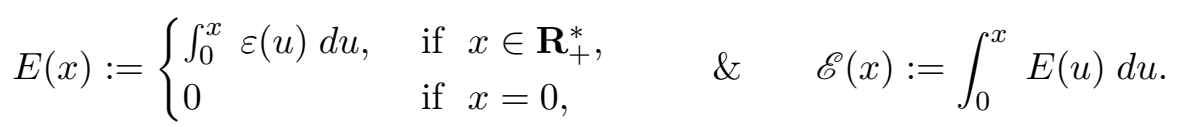

For every $\Psi$ in $\mathscr{S}^{\prime}(\mathbf{R}), \Psi^{\prime}$ will denote the derivative of $\Psi$, in the sense of tempered distribution 3 .

Theorem 3.4. The map $\Phi: \mathbf{R}_{+} \rightarrow \mathscr{S}^{\prime}(\mathbf{R})$ defined by setting:

$$
\Phi(t):=\Phi_{t}:= \begin{cases}\mathbf{1}_{[0, t)} \cdot \varepsilon(t-\cdot) & \text { if } t \in \mathbf{R}_{+}^{*}, \\ 0 & \text { if } t=0,\end{cases}
$$

fulfill Assumption $(\mathscr{A})$. More precisely, it is differentiable on $\mathbf{R}_{+}^{*}$ and, $\forall t$ in $\mathbf{R}_{+}^{*}$,

$$
\Phi^{\prime}(t):=\frac{d}{d t}[\Phi(t)]=F_{t}-\left(G_{t}\right)^{\prime}+\left(H_{t}\right)^{\prime \prime},
$$

where $F_{t}, G_{t}$ and $H_{t}$ all belong to $\mathscr{S}^{\prime}(\mathbf{R})$ and are defined by setting, $\forall t$ in $\mathbf{R}_{+}^{*}$ :

$$
\begin{aligned}
& \text { - } F_{t}:=\frac{\varepsilon(t-\cdot)}{t} \mathbf{1}_{[0, t)}+\left(\varepsilon(t)-\frac{E(t)}{t}\right) \delta_{0} ; \quad \cdot H_{t}:=\frac{((t-\cdot) \cdot E(t-\cdot)-\mathscr{E}(t-\cdot))}{t} \cdot \mathbf{1}_{[0, t)} ; \\
& \text { - } G_{t}:=\left(E(t)-\frac{\mathscr{E}(t)}{t}\right) \delta_{0}+u \mapsto\left(\frac{u \varepsilon(t-u)-E(t-u)}{t}\right) \mathbf{1}_{[0, t)}(u) .
\end{aligned}
$$

Furthermore, the map $t \mapsto\left|\Phi^{\prime}(t)\right|_{-q}$ belongs to $\underset{b \in \mathbf{R}_{+}^{*}}{\cap} L^{2}((0, b))$, for every integer $q \geq 3$.

\subsection{Generalized functionals of $G$}

In order to establish easily that the map $t \mapsto f\left(G_{t}\right)$ is $(\mathcal{S})^{*}$-integrable and integrable with respect to itself, when $f$ is function of polynomial growth, we introduce here the generalized functionals of $G$, using [Kuo96, Section 7.1]. We identify, here and in the sequel, any function $f$ of $L_{l o c}^{1}(\mathbf{R})$ with its associated tempered distribution, denoted noted $T_{f}$, when it exists. In particular, one notes in this case: $\langle f, \phi\rangle=\int_{\mathbf{R}} f(t) \phi(t) d t$, for every $\phi$ in $\mathscr{S}(\mathbf{R})$. In this latter case we say that the tempered distribution $T:=T_{f}$ is of function type. Define the sets $\mathcal{Z}_{R}:=\left\{t \in \mathscr{R} ; R_{t}=0\right\}$ and $\mathcal{Z}_{R}^{c}:=\left\{t \in \mathscr{R} ; R_{t}>0\right\}$.

\footnotetext{
${ }^{3}$ One therefore has $\left\langle\left(G_{t}\right)^{\prime}, \varphi>=-<G_{t}, \varphi^{\prime}\right\rangle$, for every $(t, \varphi)$ in $\mathbf{R}_{+}^{*} \times \mathscr{S}(\mathbf{R})$.
} 
Theorem-Definition 3.2. Let $F$ be a tempered distribution. For every $t$ in $\mathcal{Z}_{R}^{c}$, define

$$
F\left(G_{t}\right):=\frac{1}{\sqrt{2 \pi R_{t}}} \sum_{k=0}^{+\infty} \frac{1}{k ! R_{t}^{k}}<F, \xi_{t, k}>I_{k}\left(g_{t}^{\otimes k}\right),
$$

where, for every $(x, k)$ in $\mathbf{R} \times \mathbf{N}, \xi_{t, k}(x):=\pi^{1 / 4}(k !)^{1 / 2} R_{t}^{k / 2} \exp \left\{-\frac{x^{2}}{4 R_{t}}\right\} e_{k}\left(x /\left(\sqrt{2 R_{t}}\right)\right)$. Then for all real $t, F\left(G_{t}\right)$ is a Hida distribution, called generalized functional of $G_{t}$.

Proof. This is an immediate consequence of [Ku096, p.61-64], by taking $f:=g_{t}$.

Remark 2. As shown in [Ben03a], when $F=f$ is of function type, $F\left(G_{t}\right)$ coincides with $f\left(G_{t}\right)$.

The following theorem yields an estimate of $\left\|F\left(G_{t}\right)\right\|_{-p}^{2}$ which will be useful in the sequel.

Theorem 3.5. Let $p$ be in $\mathbf{N}$. Then there is a constant $D_{p}$, such that:

$$
\forall F \in \mathscr{S}_{p}(\mathbf{R}), \forall t \in \mathcal{Z}_{R}^{c}, \quad\left\|F\left(G_{t}\right)\right\|_{-p}^{2} \leq \max \left\{R_{t}^{-2 p} ; R_{t}^{2 p}\right\} R_{t}^{-1 / 2} D_{p}|F|_{-p}^{2} .
$$

Proof. This is a simple consequence of the following more general result: let $f$ be a nonzero function in $L^{2}(\mathbf{R}), p \in \mathbf{N}$ and $F \in \mathscr{S}_{-}(\mathbf{R})$. There exists a constant $D_{p}$, independent of $F$ and $f$, such that $\|F(<., f>)\|_{-p}^{2} \leq \max \left\{|f|_{0}^{-4 p} ;|f|_{0}^{4 p}\right\}|f|_{0}^{-1} D_{p}|F|_{-p}^{2}$. The line of the proof is the same as in Ben03a, Theorem 3.3] by replacing there $t^{2 H}$ by $|f|_{0}^{2}$.

\subsection{S-Transform of $G$ and $W^{(G)}$}

The following theorem makes explicit the $S$-transforms of $G$, of the Gaussian white noise $W^{(G)}$ and of generalized functionals of $G$. Denote $\gamma$ the heat kernel density on $\mathbf{R}_{+} \times \mathbf{R}$ i.e.

$$
\gamma(t, x):=\frac{1}{\sqrt{2 \pi t}} \exp \left\{\frac{-x^{2}}{2 t}\right\} \text { if } t \neq 0 \text { and } 0 \text { if } t=0 .
$$

The results provided in Theorem 3.6 below will be used a lot in the sequel, and in Section 3.4 .

Theorem 3.6. For every $\eta$ in $\mathscr{S}(\mathbf{R})$ one has the following equalities:

(i) $S\left(G_{t}\right)(\eta)=<g_{t}, \eta>_{L^{2}(\mathbf{R})}$, for every $t$ in $\mathscr{R}$,

(ii) $S\left(W_{t}^{(G)}\right)(\eta)=<g_{t}^{\prime}, \eta>=\frac{d}{d t}\left[<g_{t}, \eta>_{L^{2}(\mathbf{R})}\right]$, for every $t$ in $\mathscr{R}_{D}$;

(iii) For $p \in \mathbf{N}, F \in \mathscr{S}_{-}(\mathbf{R})$, and $t$ in $\left.\mathcal{Z}_{R}^{c}, S\left(F\left(G_{t}\right)\right)(\eta)=\left\langle F, \gamma\left(R_{t}, .-<g_{t}, \eta\right\rangle\right)\right\rangle$.

Furthermore, there exists a constant $D_{p}$, independent of $F, t$ and $\eta$, such that:

$$
\forall t \in \mathcal{Z}_{R}^{c}, \quad\left|S\left(F\left(G_{t}\right)\right)(\eta)\right|^{2} \leq \max \left\{R_{t}^{-2 p} ; R_{t}^{2 p}\right\} R_{t}^{-1 / 2} D_{p}|F|_{-p}^{2} \exp \left\{|\eta|_{p}^{2}\right\} .
$$

Proof. (i) Obvious in regard of Proposition 2.4. Point (ii) is a straightforward consequence of (iii) in Lemma 2.5, and of (3.3). The equality in (iii) results from [Kuo96, Theorem 7.3 p.63] with $f=g_{t}$. The inequality results from (3.6) as in [Ben03a, Theorem 3.8].

Before giving the general result on stochastic integral wrt $G$ we deal, in the next subsection, with Wiener integral wrt $G$. 


\subsection{Wiener integral with respect to $G$}

In all this subsection one denotes $I$ a Borel set of $\mathscr{R}$ and $f: \mathscr{R} \rightarrow \mathbf{R}$ a deterministic and measurable function We want to define the integral of $f$, on $I$, with respect to $G$. Since the map $s \mapsto G_{s}$ is (weakly) differentiable on $I$, one may think to define formally the Wiener integral wrt $G$, denoted $\int_{I} f(s) d^{\diamond} G_{s}$, by setting:

$$
\int_{I} f(s) d^{\diamond} G_{s}:=\int_{I} f(s) \cdot \frac{d G_{s}}{d s} d s=\int_{I} f(s) \cdot W_{s}^{(G)} d s,
$$

assuming $s \mapsto f(s) \cdot W_{s}^{(G)}$ is $(\mathcal{S})^{*}$-integrable on $I$. More precisely we have the following definition.

Definition 1. (Wiener integral with respect to $G$ )

For any Borel set $I$ of $\mathscr{R}$ and any deterministic measurable function $f: I \mapsto \mathbf{R}$ such that $s \mapsto f(s) W_{s}^{(G)}$ is $(\mathcal{S})^{*}$-integrable on $I$, one says that $\int_{I} f(s) d^{\diamond} G_{s}$, defined by (3.8), is the Wiener integral of $f$ on $I$, with respect to $G$, if $\int_{I} f(s) d^{\diamond} G_{s}$ belongs to $\left(L^{2}\right)$.

Even if, in practice, there will often exist an integer $q$ in $\mathbf{N}$ such that the map $t \mapsto\left|g_{t}^{\prime}\right|_{-q}$ is bounded (as it was the case in Example 3.3), it seems more than reasonable to expect, even if $t \mapsto\left|g_{t}^{\prime}\right|_{-q}$ is not bounded, that, for any finite interval $[a, b]$ of $\mathscr{R}$,

$$
\int_{(a, b)} 1 d^{\diamond} G_{s} \text { is well-defined and such that: } \int_{(a, b)} 1 d^{\diamond} G_{s}=G_{b}-G_{a}, \text { in }(\mathcal{S})^{*} .
$$

Thanks to Equality (3.8) and Theorem[3.6, it is clear that (困) entails, among other consequences, that:

- The map $s \mapsto<g_{s}^{\prime}, \eta>$ belongs to $L_{l \widetilde{c c}}^{1}(\mathscr{R})$, for every $\eta$ in $\mathscr{S}(\mathbf{R})$,

- $\int_{(a, b)}<g_{s}^{\prime}, \eta>d s=<g_{b}, \eta>-<g_{a}, \eta>$, for every $\eta$ in $\mathscr{S}(\mathbf{R})$ and $(a, b)$ in $\mathscr{R}^{2}$.

Besides, using Proposition 2.2, it is easy to establish that:

$$
|<F, \varphi>| \leq|F|_{-q}|\varphi|_{q} ; \quad \forall(F, \varphi, q) \in \mathscr{S}^{\prime}(\mathbf{R}) \times \mathscr{S}(\mathbf{R}) \times \mathbf{N} .
$$

In view of (3.11), it appears that Assumption $\mathscr{A}_{\text {(iii) }}$ is almost necessary to get (因), if one deals with Pettis integrals, and necessary if one deals with Bochner integra 4 . Moreover, and by the very definition of the space $\mathscr{S}^{\prime}(\mathbf{R})$ as the inductive limit of the sequence $\left(\mathscr{S}_{p}(\mathbf{R})\right)_{p \in \mathbf{N}}$, Assumption $\mathscr{A}_{\text {(ii) }}$ entails that, for every compact set $\mathcal{K}$ of $\mathscr{R}_{D}$, there exists an integer $q$ in $\mathbf{N}$ such that $\left|g_{t}^{\prime}\right|_{-q}<+\infty$, for every $t$ in $\mathcal{K}$. Thus $\mathscr{A}_{\text {(iii) }}$ appears to be only a slight reinforcement of $\mathscr{A}_{(\mathrm{ii})}$. Besides, it is clear that (3.10) is nothing but Assumption $\mathscr{A}_{\text {(iv) }}$. Thus, the simple considerations given in (図), as well as the ones given right above Remark[1(about $\left.\mathscr{A}_{(\mathrm{i})} \& \mathscr{A}_{(\mathrm{ii})}\right)$, entail that Assumption $(\mathscr{A})$ is almost minimal (i.e. necessary) to get a reasonable notion of Wiener integral. We will show further that these assumptions are also sufficient to provide us with a general non-anticipative stochastic integral. Denote $\mathcal{E}(\mathscr{R})$ the set of step functions on $\mathscr{R}$. We have the following property.

Proposition 3.7. For any $f$ in $\mathcal{E}(\mathscr{R}), \int_{\mathscr{R}} f(u) d^{\triangleright} G_{u}$ is a Wiener integral with respect to $G$. Moreover, let $[a, b]$ be a finite interval of $\mathscr{R}$, then $\int_{a}^{b} d^{\triangleright} G_{u}=G_{b}-G_{a}$ almost surely.

\footnotetext{
${ }^{4}$ See Appendix A.1 for precisions about Bochner integrals.
} 
Proof. Fix $\eta$ in $\mathscr{S}(\mathbf{R})$. From $(i i)$ of Theorem [3.6, $t \mapsto S\left(f(t) W_{t}^{(G)}\right)(\eta)$ is measurable on $\mathscr{R}$. Moreover we have, thanks to Lemma 2.3, $\left|S\left(f(t) W_{t}^{(G)}\right)(\eta)\right| \leq\left|g_{t}^{\prime}\right|_{-q} \sup _{t \in \mathscr{R}}|f(t)| e^{|\eta|_{-q}^{2}}$, where $q$ is the integer given by Assumption $\mathscr{A}_{\text {(iii) }}$. Theorem 2.6 then applies and entails that $f$ is $d G$ integrable on $(a, b)$. Furthermore, thanks to Lemma 2.5, one has the equality: $S\left(\int_{a}^{b} d G_{u}\right)(\eta)=$ $\int_{a}^{b} S\left(W_{u}^{(G)}\right)(\eta) d u=\left[S\left(G_{u}\right)(\eta)\right]_{a}^{b}=S\left(G_{b}-G_{a}\right)(\eta)$. The equality, in $\left(\mathcal{S}^{*}\right)$, follows from the injectivity of the S-transform. Finally, since $G_{b}-G_{a}$ belongs to $\left(L^{2}\right)$, the equality $\int_{a}^{b} d^{\diamond} G_{u}=$ $G_{b}-G_{a}$ holds in $\left(L^{2}\right)$ and hence almost surely.

The following theorem gives a sufficient condition for an integral, of the form (3.8), to be a Wiener integral. Denote $\rightsquigarrow$ the equality in law.

Theorem 3.8. Assume that there exists $q_{0}$ in $\mathbf{N}$ such that the map $s \mapsto f(s) \cdot\left|g_{s}^{\prime}\right|_{-q_{0}}$ belongs to $L^{1}(\mathscr{R})$. Then $Z:=\int_{\mathscr{R}} f(s) d^{\diamond} G_{s}$ is an element of $(\mathcal{S})^{*}$, which verifies, $Z=\sum_{k=0}^{+\infty}\left(\int_{\mathscr{R}} f(s)<g_{s}^{\prime}, e_{k}>d s\right)<., e_{k}>$ in $(\mathcal{S})^{*}$. Moreover $Z$ is a Gaussian random variable if and only if $\sum_{k=0}^{+\infty}\left(\int_{\mathscr{R}} f(s)<g_{s}^{\prime}, e_{k}>d s\right)^{2}<$ $+\infty$. In this latter case, on has:

$$
Z \rightsquigarrow \mathcal{N}\left(0, \sum_{k=0}^{+\infty}\left(\int_{\mathscr{R}} f(s)<g_{s}^{\prime}, e_{k}>d s\right)^{2}\right) .
$$

Proof. In order to show that equality $\int_{\mathscr{R}} f(s) d^{\diamond} G_{s}=\sum_{k=0}^{+\infty}\left(\int_{\mathscr{R}} f(s)<g_{s}^{\prime}, e_{k}>d s\right)<., e_{k}>$ holds in $(\mathcal{S})^{*}$, let us establish points a), b) and c) below.

a) $s \mapsto f(s) \cdot W_{s}^{(G)}$ is $(\mathcal{S})^{*}$-integrable over $\mathscr{R}$. One can use Thm. 2.6 since one has, $\forall(\eta, s)$ in $\mathscr{S}(\mathbf{R}) \times \mathscr{R}_{D}$ and using Lemma 2.3, $\left|S\left(f(s) W_{s}^{(G)}\right)(\eta)\right| \leq|f(s)|\left\|W_{s}^{(G)}\right\|_{-q_{0}} e^{|\eta|_{q_{0}}^{2}} \leq|f(s)|\left|g_{s}^{\prime}\right|_{-q_{0}} e^{|\eta|_{q_{0}}^{2}}$. b) $\Psi_{f}:=\sum_{k=0}^{+\infty}\left(\int_{\mathscr{R}} f(s)<g_{s}^{\prime}, e_{k}>d s\right)<., e_{k}>$ belongs to $\left(\mathcal{S}_{-p_{0}}\right)$, as soon as $p_{0} \geq q_{0}+1$. Let $p_{0}$ be in $\mathbf{N}$ such that $p_{0} \geq q_{0}+1$. Recall the definition of $\mathcal{R}_{n}$ given in (3.4). Proposition 2.2 and (3.11) entail that $\left\|\Psi_{f}\right\|_{-p_{0}}^{2} \leq\left\|s \mapsto f(s) \cdot\left|g_{s}^{\prime}\right|_{-q_{0}}\right\|_{L^{1}(\mathscr{R})}^{2} \mathcal{R}_{p_{0}-q_{0}}<+\infty$.

c) $\Phi_{f}:=\int_{\mathscr{R}} f(s) d^{\diamond} G_{s}$ is equal to $\Psi_{f}$ in $(\mathcal{S})^{*}$. Define the $(\mathcal{S})^{*}$-process $\tau$ and the family of $(\mathcal{S})^{*}$-processes $\left(\tau_{N}\right)_{N \in \mathbf{N}}$ by setting, for every real $\left.s, \tau(s):=\sum_{k=0}^{+\infty} f(s)<g_{s}^{\prime}, e_{k}\right\rangle\left\langle., e_{k}\right\rangle$, and $\tau_{N}(s):=\sum_{k=0}^{N} f(s)<g_{s}^{\prime}, e_{k}><., e_{k}>$. Obviously we have $\Phi_{f}=\int_{\mathscr{R}} \tau(s) d s, \Psi_{f}=$ $\lim _{N \rightarrow+\infty} \int_{\mathscr{R}} \tau_{N}(s) d s$ in $(\mathcal{S})^{*}$. It then remains to show that $\Phi_{f}=\lim _{N \rightarrow+\infty} \int_{\mathscr{R}} \tau_{N}(s) d s$ in $(\mathcal{S})^{*}$. For this purpose, we use Theorem A.2. Let $\left(p_{0}, n\right)$ be a couple of integers with $p_{0} \geq q_{0}+1$. It is easily seen that $\tau_{N}$ and $\tau$ are weakly measurable on $\mathscr{R}$ (see Definition 3 ) and, using the same upper-bound we used in b), that $\tau_{N}(s)$ and $\tau(s)$ belong to $\left(\mathcal{S}_{-p_{0}}\right)$ for every real $s$. Moreover, using Proposition 2.2 and, again, the upper-bound we used in $\mathbf{b}$ ), it is clear that both functions $s \mapsto\left\|\tau_{N}(s)\right\|_{-p_{0}}$ and $s \mapsto\|\tau(s)\|_{-p_{0}}$ belong to $L^{1}(\mathscr{R}, d s)$ since $\left\|\tau_{N}(s)\right\|_{-p_{0}}^{2} \leq$ $\|\tau(s)\|_{-p_{0}}^{2} \leq f^{2}(s)\left|g_{s}^{\prime}\right|_{-q_{0}}^{2} \mathcal{R}_{p_{0}-q_{0}}$. We hence have shown that both functions $\tau_{N}($.$) and \tau($.$) are$ Bochner integrable on $\mathscr{R}$. Besides, for every $(n, m)$ in $\mathbf{N}^{2}$ with $n \geq m$, we have, thanks to the previous upper bound, $\int_{\mathscr{R}}\left\|\tau_{n}(s)-\tau_{m}(s)\right\|_{p_{0}} d s \leq \int_{\mathscr{R}}\left\|_{k=m+1}^{+\infty} f(s)<g_{s}^{\prime}, e_{k}><., e_{k}>\right\|_{-p_{0}} d s \leq$ $\mathcal{R}_{p_{0}-q_{0}} \cdot\left\|s \mapsto f(s)\left|g_{s}^{\prime}\right|_{-q_{0}}\right\|_{L^{1}(\mathscr{R})}$. It is then clear that the left hand side of the previous inequality tends to 0 as $(n, m)$ tends to $(+\infty,+\infty)$. Theorem A.2 (see Appendix A.1) applies and establishes c). Finally, $Z$ is the $\left(L^{2}\right)$-limit of a sequence of independent Gaussian variables if $\sum_{k=0}^{+\infty}\left(\int_{\mathscr{R}} f(s)<g_{s}^{\prime}, e_{k}>d s\right)^{2}<+\infty$. The equality $\mathbf{E}\left[Z^{2}\right]=\sum_{k=0}^{+\infty}\left(\int_{\mathscr{R}} f(s)<g_{s}^{\prime}, e_{k}>d s\right)^{2}$ then becomes obvious.

Example 3.9. 1. If $G$ is a Brownian motion, point 1 of Example 3.3. Theorem 3.8 as well as the equality $\sum_{k=0}^{+\infty}\left(\int_{\mathbf{R}} f(s)<\delta_{s}, e_{k}>d s\right)^{2}=\|f\|_{L^{2}(\mathbf{R})}^{2}$, allow us to define the Wiener integral 
of $f$, in sense of Definition 1, for any $f$ in $L^{2}(\mathbf{R})$. This shows that our definition of Wiener integral wrt Brownian motion and the usual one both coincide exactly. Besides, it is clear that $I_{f}:=\int_{0}^{1} f(s) \diamond B_{1} d s$ is an $\left(L^{2}\right)$ random variable if and only if $f$ belongs to $L^{2}([0,1])$. Therefore Theorem [2.6 allows us to define the Wiener integral of $f$ wrt Brownian bridge, in sense of Definition 1, if and only if $f$ belongs to $L^{2}([0,1])$.

2. The case of Wiener integral wrt fBm (resp. wrt mBm) has been treated in LLV14, Section 4] (resp. in [LLV14, Sections 2.384 4]). In view of, the previous point of this example, Example 3.3 and Theorem [3.8, one can extend [LLV14, Proposition 4.31] and claim that $\int_{\mathbf{R}} f(s) d^{\diamond} B_{s}^{H}$ is the Wiener integral of $f$, wrt $B^{H}$, for every function $f$ in $L^{1}(\mathbf{R}) \cap L_{H}^{2}(\mathbf{R})$, where $L_{H}^{2}(\mathbf{R})$ has been defined in Subsection 2.4. The fonctions for which one can defined a Wiener integral wrt $m B m$ are included into $\overline{\mathcal{E}(\mathbf{R})}{ }^{2,>_{h}}$, where $<,>_{h}$ denotes the inner product, defined (in LLV14, Sections 2.3 E Proposition 3.1.]) by setting $\left\langle\mathbf{1}_{[0, t]}, \mathbf{1}_{[0, s]}\right\rangle_{h}=R_{h}(t, s)$, and where $R_{h}$ has been defined in (2.3).

3. In the case of $V_{\gamma}$ - processes, one can improve Proposition 14 of [MV05]. Indeed, denote $\mathcal{H}$ the set of all functions for which [MV05, Section 3] define a Wiener integral. For any $\eta$ : $[0, T] \rightarrow \mathbf{R}_{+}$, continuous and increasing in a neighborhood of 0 and such that $\lim _{0+} \eta=0$, define the set $\mathbf{C}^{\eta}:=\left\{f \in L^{2}([0, T]), \sup _{0 \leq r<s \leq T}|f(s)-f(r)| / \eta(s-r)<+\infty\right\}$. In order to show that $\mathcal{H}$ contains $\mathbf{C}^{\eta}$, [MV05, Proposition 14] has to require an additional assumption on $\eta$. No such assumption is required here. Using only Theorem 3.8, one easily sees that, for every $T>0$, the process $\left(Z_{t}\right)_{t \in[0, T]}$, defined by $Z_{t}:=\int_{0}^{t} f(s) d^{\triangleleft} G_{s}$, where $f$ belongs to $\mathbf{C}^{\eta}$, is a Gaussian process. One just needs to see that, $|f(s)| \cdot\left|\Phi^{\prime}(s)\right|_{-q_{0}} \leq\left(M+\left|f\left(r_{0}\right)\right|\right)\left(1+\left|\Phi^{\prime}(s)\right|_{-q_{0}}\right)$, for every $(f, s)$ in $\mathbf{C}^{\eta} \times[0, T]$, where $M:=\sup _{0 \leq u \leq T} \eta(u) \cdot \sup _{0 \leq r<s \leq T}|f(s)-f(r)| / \eta(s-r)$, $\Phi$ and $\Phi^{\prime}$ have been defined in Theorem 3.4 and $r_{0}$ is any real in $(0, T]$ such that $\left|f\left(r_{0}\right)\right|<+\infty$. Using Theorem 3.4, one concludes that $s \mapsto|f(s)| \cdot\left|\Phi^{\prime}(s)\right|_{q_{0}}$ belongs to $L^{1}((0, T))$ and one then uses Thm. 3.8

Remark 3. In fact one can extend the notion of Wiener integral wrt any $G$ in $\mathscr{G}$ in two ways. The first way, which is also the more general one, is given in Point 1. of Remark 10. The second way it is the following: If the bilinear form $<,>_{R}$, defined on $\mathcal{E}(\mathscr{R}) \times \mathcal{E}(\mathscr{R})$ by setting $\left.<\mathbf{1}_{[0, t]}, \mathbf{1}_{[0, s]}\right\rangle_{R}:=R(t, s)$ is an inner product; assuming there exists an isometry, denoted $M:\left(\mathcal{E}(\mathscr{R}),<,>_{R}\right) \rightarrow\left(L^{2}(\mathbf{R}),<,>_{L^{2}(\mathbf{R})}\right)$, such that $g_{t}:=M\left(\mathbf{1}_{[0, t]}\right)$, then one can extend the notion of Wiener integral to any elements of $\overline{\mathcal{E}(\mathscr{R})}<>_{R}$. This latter space contains in general not only functions but also tempered distributions. This general method applies to fBm and $\mathrm{mBm}$ (see [LLV14, Section 3]), as well as to Volterra processes.

Remark 4. As it is explained in [SV14, Example 3.3.] the Brownian bridge admits several representations, among which are the orthogonal one, the Fredholm one and the canonical one. It is clear that both the orthogonal and the canonical representations of the Brownian bridge on $[0, T]$ fulfill Assumption $[\mathscr{D})$ on $\mathscr{R}:=[0, T]$ (since there exists $q$ in $\mathbf{N}^{*}$ such that $t \mapsto\left|g_{t}^{\prime}\right|_{-q}$ belongs to $L^{1}([0, b], d t)$, for every $b$ in $[0, T)$. This result can be extended to Gaussian bridges (see [GSV07] for more details about this latter notion), assuming the Gaussian process $G:=$ $\left(G_{t}\right)_{t \in[0, T]}$ fulfills Assumption $(\mathscr{A})$.

\subsection{The Wick-Itô integral with respect to Gaussian processes}

We still assume in this section that Assumption $(\mathscr{A})$ holds. We are now able to define, and give the main properties, of the Wick-Itô integral wrt $G$. We still denote $I$ a Borel set of $\mathscr{R}$ and let $X:=\left(X_{t}\right)_{t \in \mathscr{R}}$ be an $(\mathcal{S})^{*}$-valued process. Because the belonging to $(\mathcal{S})^{*}$ is not stable by ordinary product, one can not generalize (3.8) to any $(\mathcal{S})^{*}$-valued process $X$, by simply setting: 


$$
\int_{I} X_{s} d^{\diamond} G_{s}:=\int_{I} X_{s} \cdot \frac{d G_{s}}{d s} d s=\int_{I} X_{s} \cdot W_{s}^{(G)} d s
$$

However, since the belonging to $(\mathcal{S})^{*}$ is stable by Wick product one may extend the integral $(3.8)$ to $(\mathcal{S})^{*}$-valued processes $X$ in the following manner.

Definition 2 (Wick-Itô integral wrt Gaussian process). Let $X: \mathscr{R} \rightarrow(\mathcal{S})^{*}$ be a process such that the process $t \mapsto X_{t} \diamond W_{t}^{(G)}$ is $(\mathcal{S})^{*}$-integrable on $\mathscr{R}$. The process $X$ is then said to be $d G$ integrable on $\mathscr{R}$ (or integrable on $\mathscr{R}$ ), wrt the Gaussian process $G$. The Wick-Itô integral of $X$ wrt $G$, on $\mathscr{R}$, is defined by setting:

$$
\int_{\mathscr{R}} X_{s} d^{\diamond} G_{s}:=\int_{\mathscr{R}} X_{s} \diamond W_{s}^{(G)} d s .
$$

For any Borel set $I$ of $\mathscr{R}$, define $\int_{I} X_{s} d^{\diamond} G_{s}:=\int_{\mathscr{R}} \mathbf{1}_{I}(s) X_{s} d^{\diamond} G_{s}$.

The Wick-Itô integral of an $\left(\mathcal{S}^{*}\right)$-valued process, wrt $G$ is then an element of $(\mathcal{S})^{*}$. It is easy to see that Wick-Itô integration wrt $G$, is linear and that Definition 2 is coherent with Definition 1. of Wiener integral, we gave in the previous subsection. Moreover, and as it will be stated in Proposition 3.11 below, one of the advantages of Definition 2 is that our integral wrt $G$ is centered, assuming it belongs to $\left(L^{2}\right)$. Used a lot in the sequel of this paper, the following condition ensures the integrability, on $I$, of an $(\mathcal{S})^{*}$-valued process $X$, wrt $G$.

Let $X: I \rightarrow(\mathcal{S})^{*}$ be an $(\mathcal{S})^{*}$-valued process. Denote the following condition:

$(\mathcal{I})\left\{\begin{array}{l}(i): \quad t \mapsto S\left(X_{t}\right)(\eta) \text { is measurable on } I, \text { for all } \eta \text { in } \mathscr{S}(\mathbf{R}) \\ (i i): \quad \exists(p, q) \in \mathbf{N}^{2} \text { such that the map } t \mapsto\left\|X_{t}\right\|_{-p}\left\|W_{t}^{(G)}\right\|_{-q} \text { belongs to } L^{1}(I, d t) .\end{array}\right.$

When the processes $X$ and $G$ satisfy condition $(\mathcal{I})$ on $I$, we will say that $(X, G)$ satisfies $(\mathcal{I})$ or $\left(\overline{\left.\mathcal{I}_{p, q}\right)}\right.$, when we want to specify the value of $p$ and $q$ in $(i i)$ of Condition $(\mathcal{I})$. We will use the following theorem a lot in the sequel.

Theorem 3.10. If $(X, G)$ satisfies condition $\left(\mathcal{I}_{p, q}\right)$ on $I$, then $\int_{I} X_{s} d^{\diamond} G_{s}$ is well-defined and belongs to $\left(\mathcal{S}_{-r}\right)$ for every $r \geq 2+\max \{p ; q\}$. Moreover there exists a real constant $C$, independent of $X$ and $G$, such that:

$$
\forall r \geq 2+\max \{p ; q\} ; \quad\left\|\int_{I} X_{t} d^{\diamond} G_{t}\right\|_{-r} \leq C \int_{I}\left\|X_{t}\right\|_{-p}\left\|W_{t}^{(G)}\right\|_{-q} d t .
$$

Proof. $\forall \eta \in \mathscr{S}(\mathbf{R})$, the measurability on $I$ of $t \mapsto S\left(X_{t} \diamond W_{t}^{(G)}\right)(\eta)$ is clear since $S\left(X_{t} \diamond\right.$ $\left.W_{t}^{(G)}\right)(\eta)=S\left(X_{t}\right)(\eta)<g_{t}^{\prime}, \eta>$. Condition (讘) being verified, we use Lemma 2.3 to get, for

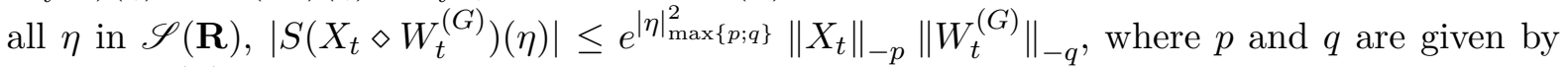
condition (I) . Theorem 2.6 then clearly applies. The upper-bound in the theorem, as well as the existence of $r$ and $C$, results from [Kuo96, Thm. 13.5].

We can now give the first properties of the Wick-Itô integral wrt $G$.

Proposition 3.11. (i) Let $I$ be a Borel subset of $\mathscr{R}$ and $X: I \rightarrow(\mathcal{S})^{*}$ a $d G$-integrable process over I. Assume that $\int_{I} X_{s} d^{\diamond} G_{s}$ belongs to $\left(L^{2}\right)$. Then $\mathbf{E}\left[\int_{I} X_{s} d^{\diamond} G_{s}\right]=0$.

(ii) Let $[a, b] \subset \mathscr{R}$. The $\left(\mathcal{S}^{*}\right)$-process $\Psi$ defined by $\Psi(t):=\int_{a}^{t} X_{s} d^{\diamond} G_{s}$ is continuous on $[a, b]$, as soon as $(X, G)$ satisfies condition (II) on $[a, b]$. 
Proof. (i) That $S\left(\int_{I} X_{s} d^{\diamond} G_{s}\right)(0)=\int_{I} S\left(X_{s}\right)(0) S\left(W_{s}^{(G)}\right)(0) d s=0$ is clear since $S\left(W_{s}^{(G)}\right)(0)=$ $<g_{s}^{\prime}, 0>=0$. Now, it sufficient to note that $\mathbf{E}[U]=S(U)(0)$ for every r.v. $U$ in $\left(L^{2}\right)$.

(ii) The integrability of $X$ wrt $G$ is proved by Theorem 3.10, Let $t_{0}$ be fixed in $(a, b)$. In order to establish the continuity of $\Psi$ in $t_{0}$ we are going to use [Kuo96, Theorem 8.6]. By symmetry one may assume that $t_{0} \geq t$. [Kuo96, Theorem 8.6] applies since we clearly have:

a) $\left|S\left(\Psi(t)-\Psi\left(t_{0}\right)\right)(\eta)\right| \leq e^{|\eta|_{\max \{p ; q\}}^{2}} \int_{t}^{t_{0}}\left\|X_{u}\right\|_{-p}\left\|W_{u}^{(G)}\right\|_{-q} d u \underset{t \rightarrow t_{0}}{\longrightarrow} 0$;

b) $|S(\Psi(t))(\eta)| \leq e^{|\eta|_{\max \{p ; q\}}^{2}} \int_{[a, b]}\left\|X_{u}\right\|_{-p}\left\|W_{u}^{(G)}\right\|_{-q} d u$.

Proposition 3.12. Let $(X, G)$ be a couple of processes that satisfies condition (II) on $\mathscr{R}$. Define, for every $n$ in $\mathbf{N}$, the process $G^{(n)}:=\left(G_{t}^{(n)}\right)_{t \in \mathscr{R}}$ by setting $G_{t}^{(n)}:=<., g_{t}^{(n)}>$, where $g_{t}^{(n)}$ belongs to $L^{2}(\mathbf{R})$ and let $\left(X^{(n)}\right)_{n \in \mathbf{N}}:=\left\{\left(X_{t}^{(n)}\right)_{t \in \mathscr{R}} ; n \in \mathbf{N}\right\}$ denote a sequence of $(\mathcal{S})^{*}$-valued processes. Let us write the following conditions:

$\left.a_{1}\right)\left(X, G^{(n)}\right)$ satisfies condition $(\underline{\mathcal{I}})$ on $I$, uniformly $y^{5}$ in $q$.

$\left.a_{2}\right) \exists\left(r, r_{1}\right) \in \mathbf{N} \times(0,+\infty]$ such that: $\left\|G^{(n)}-G .\right\|_{-r} \underset{n \rightarrow+\infty}{\longrightarrow} 0$, where the convergence holds both pointwisely on $I$, and in $L^{r_{1}}(I)$,

$\left.a_{3}\right) X$ is $(\mathcal{S})^{*}$-differentiable on $I$ and there exist $(a, l) \in \mathbf{N} \times \mathbf{R}$ and a function $L \in L^{r_{2}}(I, d t)$ s.t.

$$
\left|\frac{d}{d s}\left[S\left(X_{s}\right)(\eta)\right]\right| \leq L(s) e^{a|\eta|_{l}^{2}},
$$

for all $\eta$ of $\mathscr{S}(\mathbf{R})$ and for a.e. $s$ of $I$, where $r_{2} \in(0,+\infty]$ is such that $r_{1}^{-1}+r_{2}^{-1}=1$,

$\left.a_{2}^{\prime}\right) \exists\left(r_{1}, r_{2}\right) \in(0,+\infty]^{2}$ with $r_{1}^{-1}+r_{2}^{-1}=1$, such that:
(i) $\left\|s \mapsto\left|g_{s}^{\prime(n)}-g_{s}^{\prime}\right|_{-q}\right\|_{L^{r_{1}(I)}} \underset{n \rightarrow+\infty}{\longrightarrow}$;
(ii) $s \mapsto\left\|X_{s}\right\|_{-p}$ belongs to $L^{r_{2}}(I)$.

If conditions $\left(a_{i}\right)_{i \in\{1 ; 2 ; 3\}}$ or both conditions $\left(a_{1}\right)$ and $\left(a_{2}^{\prime}\right)$ are fulfilled, then one has the convergence:

$$
\int_{I} X_{s} d^{\diamond} G_{s}^{(n)} \underset{n \rightarrow+\infty}{\longrightarrow} \int_{I} X_{s} d^{\diamond} G_{s} \quad \text { in }(\mathcal{S})^{*}
$$

Besides, denote the following conditions:

$\left.b_{1}\right)\left(X^{(n)}, G\right)$ satisfies condition (I) on $I$, uniformly in $p$.

$\left.b_{2}\right) \exists r \in \mathbf{N}$ such that: $\left\|X^{(n)}-X\right\|_{-r} \underset{n \rightarrow+\infty}{\longrightarrow} 0$ pointwise.

$\left.b_{3}\right)$ Both $X$ and $\left(X^{(n)}\right)_{n \in \mathbf{N}}$ are $(\mathcal{S})^{*}$-differentiable on I. Moreover, $\exists\left(l, a, r_{2}\right) \in \mathbf{N} \times \mathbf{R} \times(0,+\infty]$ and a function $L$ in $L^{r_{2}}(I, d t)$ such that, for every $(n, \eta)$ in $\mathbf{N} \times \mathscr{S}(\mathbf{R})$ and a.e. $s \in I$,

$$
\left|\frac{d}{d s}\left[S\left(X_{s}^{(n)}\right)(\eta)\right]\right|+\left|\frac{d}{d s}\left[S\left(X_{s}\right)(\eta)\right]\right| \leq L(s) e^{a|\eta|_{l}^{2}}
$$

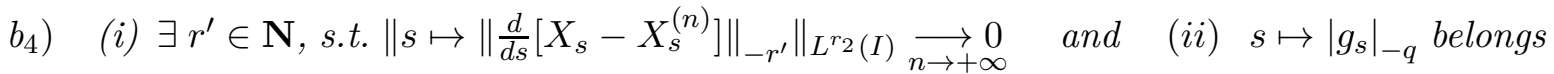
to $L^{r_{1}}(I)$, where $r_{1} \in(0,+\infty]$ is such that $r_{1}^{-1}+r_{2}^{-1}=1$.

$\left.b_{2}^{\prime}\right) \exists\left(r_{1}, r_{2}\right) \in(0,+\infty]^{2}$ with $r_{1}^{-1}+r_{2}^{-1}=1$, such that:
(i) $\|s \mapsto\| X_{s}-X_{s}^{(n)}\left\|_{-p}\right\|_{L^{r_{2}(I)}} \underset{n \rightarrow+\infty}{\longrightarrow} 0$ to $L^{r_{2}}(I)$
(ii) $s \mapsto\left|g_{s}^{\prime}\right|_{-q} \in L^{r_{1}}(I)$.

\footnotetext{
${ }^{5}$ i.e. $\exists(p, q) \in \mathbf{N}^{2}$, such that $s \mapsto\left\|X_{s}\right\|_{-p}\left\|W_{s}^{\left(G^{(n)}\right)}\right\|_{-q}$ belongs to $L^{1}(I, d s)$, for every $n$ in $\mathbf{N}$.
} 
If conditions $\left(b_{i}\right)_{i \in\{1 ; 2 ; 3 ; 4\}}$ or both conditions $\left(b_{1}\right)$ and $\left(b_{2}^{\prime}\right)$ are fulfilled, then one has the convergence:

$$
\int_{I} X_{s}^{(n)} d^{\diamond} G_{s} \underset{n \rightarrow+\infty}{\longrightarrow} \int_{I} X_{s} d^{\diamond} G_{s}, \quad \text { in }(\mathcal{S})^{*} .
$$

Proof. The scheme of the proof is "symmetric in $G^{(n)}$ and $X^{(n)}$ "; we will then only show the convergence $\int_{I} X_{s} d^{\diamond} G_{s}^{(n)} \underset{n \rightarrow+\infty}{\longrightarrow} \int_{I} X_{s} d^{\diamond} G_{s}$. Denote $A_{n}:=\int_{I} X_{s} d^{\diamond} G_{s}-\int_{I} X_{s} d^{\diamond} G_{s}^{(n)}$; let us show that assumptions of [Kuo96, Theorem 8.6] are fulfilled. The existence of all following integrals come form Theorem 2.6.

- Case where $\left.a_{1}\right) \& a_{2}^{\prime}$ ) are fulfilled:

$\left|S\left(A_{n}\right)(\eta)\right|^{r_{1} r_{2}}=\left(\left|\int_{I} S\left(X_{s}\right)(\eta) S\left(W_{s}^{\left(G^{(n)}\right)}-W_{s}^{(G)}\right)(\eta) d s\right|\right)^{r_{1} r_{2}} \leq\left(\int_{I}\left\|X_{s}\right\|_{-p}^{r_{2}} d s\right)\left(\int_{I}\left|g_{s}^{\prime(n)}-g_{s}^{\prime}\right|_{-q}^{r_{1}} d s\right.$.

- Case where $\left(a_{i}\right)_{i \in\{1 ; 2 ; 3\}}$ are fulilled : Let us assume that $I=[0, t]$. An integration by parts yields:

$$
\begin{aligned}
\left|S\left(A_{n}\right)(\eta)\right| & =\left|\int_{I} S\left(X_{s}\right)(\eta) S\left(W_{s}^{\left(G^{(n)}\right)}-W_{s}^{(G)}\right)(\eta) d s\right|=\left|\int_{I} S\left(X_{s}\right)(\eta)<\left(g_{\cdot}^{(n)}\right)^{\prime}{ }_{s}-g_{s}^{\prime}, \eta>d s\right| \\
& =<g_{t}^{(n)}-g_{t}, \eta>S\left(X_{t}\right)(\eta)-\int_{I}<g_{s}^{(n)}-g_{s}, \eta>\frac{d}{d s}\left[S\left(X_{s}\right)(\eta)\right] d s \\
& \leq e^{(1+a)|\eta|_{(p+r+l)}^{2}}\left(\left\|X_{t}\right\|_{-p}\left\|G_{t}^{(n)}-G_{t}\right\|_{-r}+\int_{I}\left\|G_{s}^{(n)}-G_{s}\right\|_{-r} L(s) d s\right) .
\end{aligned}
$$

The Hölder inequality then allows one to establish the two conditions of [Kuo96, Theorem 8.6] and therefore achieves the proof.

Remark 5. 1. The advantage of condition (I) is that it allows us to make assumptions on both elements of the couple $(X, G)$ instead of making assumptions only on $X$ or only on $G$. Thus, the more informations on the "regularity" of $X$ (resp. of $G$ ) one gets, the less informations one needs on the "regularity" of $G$ (resp. of $X$ ).

2. It is clear, in $a_{2}$ ) of (iii) of Proposition [3.12, that one can also choose the pointwise convergence in $\left(L^{2}\right)$ or in probability instead of convergence in $\left(\mathcal{S}_{-r}\right)$.

3. When $G$ is an fBm (resp. an $m B m$ ), the Wick-Itô integral wrt $G$ given by Definition 2 is nothing but the fractional (resp. multifractional) Wick-Itô integral defined in [EVdH03, BSØW04, Ben03a] (resp. in [LLV14, LLVH14, Leb13]).

It is of interest to have also a criterion of integrability for generalized functionals of $G$. This will provide a very simple proof of the fact that both $\int_{a}^{b} f\left(G_{t}\right) d t$ and $\int_{a}^{b} f\left(G_{t}\right) d^{\triangleright} G_{t}$ exist in $(\mathcal{S})^{*}$.

Theorem 3.13. Let $p$ be in $\mathbf{N},[a, b]$ be an interval of $\mathcal{Z}_{R}^{c}$ and let $F$ be in $\mathscr{S}_{-}(\mathbf{R})$. If $t \mapsto$ $\max \left\{R_{t}^{-p-1 / 4} ; R_{t}^{p-1 / 4}\right\}$ belongs to $L^{1}([a, b])$ (resp. there exists an integer $q$ such that the map $t \mapsto\left|g_{t}^{\prime}\right|_{-q} \max \left\{R_{t}^{-p-1 / 4} ; R_{t}^{p-1 / 4}\right\}$ belongs to $\left.L^{1}([a, b])\right)$, then the stochastic distribution process $F\left(G_{t}\right)$ is $(\mathcal{S})^{*}$-integrable (resp. dG-integrable) on $[a, b]$ (resp. on $(a, b)$ ).

Proof. Lemma 2.3 and Equality (3.6) both apply and allow us to use Theorem 2.6 .

Remark 6. Of course conditions of Theorem 3.13 are obviously verified when the infimum of $t \mapsto R_{t}$ on $[a, b]$ is positive and when its supremum is upper-bounded on $[a, b]$. Moreover, in the particular case where these latter conditions hold, Theorem 3.13 entails that both quantities $\int_{a}^{b} f\left(G_{t}\right) d t$ and $\int_{a}^{b} f\left(G_{t}\right) d^{\triangleright} G_{t}$ exist in $(\mathcal{S})^{*}$, as soon as $f$ is a function of polynomial growth.

Example 3.14 (Computation of $\int_{0}^{T} G_{t} d^{\diamond} G_{t}$ ). Let $T>0$. Assume that $[0, T] \subset \mathscr{R}$ and that $t \mapsto R_{t}$ is upper-bounded on $[0, T]$, then the following equality holds almost surely and in $\left(L^{2}\right)$.

$$
\int_{0}^{T} G_{t} d^{\triangleright} G_{t}=\frac{1}{2}\left(G_{T}^{2}-R_{T}\right)
$$


This result will be obtained as a direct consequence of Itô formulas provided in Section 4. The direct proof is therefore left to the reader.

Remark 7. In the previous example, we could have replaced the assumption $t \mapsto R_{t}$ is upperbounded on $[0, T]$ by $\int_{0}^{T} R_{t} \cdot\left|g_{t}^{\prime}\right|_{-q} d t<+\infty$.

To end this section, we present a simple but classical stochastic differential equation, driven by a Gaussian process. We need first to generalize the definition of the Wick exponential, given at the beginning of Subsection 2.3, to the case where $\Phi$ belongs to $(\mathcal{S})^{*}$. For any $\Phi$ in $(\mathcal{S})^{*}$ and $k$ in $\mathbf{N}^{*}$ let $\Phi^{\diamond k}$ denotes the Wick product of $\Phi$, taken $k$ times. For any $\Phi$ in $(\mathcal{S})^{*}$ such that the sum $\sum_{k=0}^{+\infty} \frac{\Phi^{\diamond k}}{k !}$ converges in $(\mathcal{S})^{*}$, define the Wick-exponential of $\Phi$, and denote $\exp ^{\diamond} \Phi$, the element of $(\mathcal{S})^{*}$ defined by $\exp ^{\diamond} \Phi:=\sum_{k=0}^{+\infty} \frac{\Phi^{\diamond k}}{k !}$. For $f$ in $L^{2}(\mathbf{R})$ and $\Phi:=<., f>$, it is easy to verify that $\exp ^{\diamond} \Phi=: e^{<, f>}:$.

Example 3.15 (The Gaussian Wick exponential). Let $\mathscr{R}=\mathbf{R}_{+}$and let us consider the following Gaussian SDE:

$$
(E):\left\{\begin{aligned}
d X_{t} & =\alpha(t) X_{t} d t+\beta(t) X_{t} d^{\diamond} G_{t} \\
X_{0} & \in(\mathcal{S})^{*},
\end{aligned}\right.
$$

where $\alpha: \mathbf{R}_{+} \rightarrow \mathbf{R}$ and $\beta: \mathbf{R}_{+} \rightarrow \mathbf{R}$ are two deterministic continuous functions. Of course $(E)$ is a shorthand notation for $X_{t}=X_{0}+\int_{0}^{t} \alpha(s) X_{s} d s+\int_{0}^{t} \beta(s) X_{s} d^{\diamond} G_{s}$. As in [HOUZ10], it is easy to guess the solution. Let us define the process $Z$ by setting:

$$
Z_{t}:=X_{0} \diamond \exp ^{\diamond}\left(\int_{0}^{t} \alpha(s) d s+\int_{0}^{t} \beta(s) d^{\diamond} G_{s}\right), \quad t \in \mathbf{R}_{+},
$$

Theorem 3.16. The process $Z$ defined by (3.15) is the unique solution, in $(\mathcal{S})^{*}$, of $(E)$.

Proof. This is a straightforward application of [HOUZ10, Theorem 3.1.2].

\section{Itô Formula}

The main result of this section is Theorem 4.4, which provides an Itô Formula in $\left(L^{2}\right)$, for $C^{1,2}$ functions, with sub-exponential growth. This latter result is given in Subsection 4.1, while the end of this section is devoted to a complete comparison between our Itô formula and all the Itô formulas for Gaussian processes provided so far in the literature of functional extensions of itô integral that are: AMN01, Thms 1 \& 2], [MV05, Thm 31], [NT06, Thm 1], [KRT07, Cor. 8.13], KR10, Prop. 11.7], [LN12, Thm. 3.2]. It will, in particular, show the generality of the Itô formula for Gaussian processes of the form (1.1) we establish here.

Let us first recall a few basic facts about Lebesgue-Stieljes \& Riemann-Stieljes integrals, that will be used extensively in the remaining part of this work. Let $[a, b]$ be an interval of $\mathbf{R}$ and $j:[a, b] \rightarrow \mathbf{R}$ be a function of bounded variation. Denote $\alpha_{j}$ the signed measure such that $j(t)=\alpha_{j}([a, t])$, for every $t$ in $[a, b]$. For any function $f:[a, b] \rightarrow \mathbf{R}$, denote $\int_{a}^{b} f(s) d j(s)$ or $\int_{a}^{b} f(s) d \alpha_{j}(s)$ the Lebesgue-Stieljes integral of $f$ with respect to $j$, assuming it exists. In this latter case, we will write that $f \in L^{1}(I, d j(t))$ or $L^{1}\left(I, \alpha_{j}\right)$. In the particular case where the function $f$ is continuous on $[a, b]$, the Lebesgue-Stieljes integral of $f$ exists and is also equal to the Riemann-Stieljes integral of $f$, which is denoted and defined by:

$$
\text { (R.S.) } \int_{a}^{b} f(s) d j(s):=\lim _{\pi \rightarrow 0} \sum_{i=1}^{n} f\left(\xi_{i}^{(n)}\right)\left(j\left(x_{i}\right)-j\left(x_{i-1}\right)\right) \text {, }
$$


where the convergence holds uniformly on all finite partitions $\mathscr{P}_{\pi}^{(n)}:=\left\{a:=x_{0} \leq x_{1} \leq \cdots \leq\right.$ $\left.x_{n}:=b\right\}$ of $[a, b]$ such that $\max _{1 \leq i \leq n}\left(x_{i}-x_{i-1}\right) \leq \pi$ and such that $\xi_{i}^{(n)}$ belongs to $\left[x_{i-1}, x_{i}\right]$. The following result, will be used extensively in the sequel of this section.

Lemma 4.1. Let $[a, b]$ be a finite interval of $\mathbf{R}, I$ (resp. $J$ ) an interval of $\mathbf{R}_{+}$(resp. of $\mathbf{R}$ ) and let $L:[a, b] \times I \times J$ be $a C^{1}$-function. Let $f:[a, b] \rightarrow I$ and $j:[a, b] \rightarrow J$ be two continuous functions of bounded variation on $[a, b]$. Then one has the following equality:

$$
\begin{aligned}
L(b, f(b), j(b))-L(a, f(a), j(a)) & =\int_{a}^{b} \frac{\partial L}{\partial u_{1}}(s, f(s), j(s)) d s+\int_{a}^{b} \frac{\partial L}{\partial u_{2}}(s, f(s), j(s)) d f(s) \\
& +\int_{a}^{b} \frac{\partial L}{\partial u_{3}}(s, f(s), j(s)) d j(s) .
\end{aligned}
$$

Proof. All the integrands in the right hand side of (4.2) are continuous. Thus the LebesgueStieljes integrals in the right hand side of (4.2) are also Riemann-Stieljes integrals. It is then easy to deduce (4.2), using (4.1).

In view of Theorem-Definition 2.1, it is clear that we can extend the notion of integral in $(\mathcal{S})^{*}$ to the case where $m$ is a signed measure (the notation remaining the same). We will therefore keep the same notations for this integral, whatever the measure $m$ is (signed or positive). In the remaining of this paper, and unless otherwise specify, the measure $m$ denote a measure, that may be $\sigma$-finite or signed.

\subsection{Itô Formula in $\left(L^{2}\right)$ for $C^{1,2}$ functions with sub-exponential growth}

Let us begin with the following lemma, the proof of which is an immediate consequence of Wid44, Theorems 1,2 p.88-89].

Lemma 4.2. Let $T>0$ and $v:[0, T] \times \mathbf{R} \rightarrow \mathbf{R}$ be a continuous function such that there exists a couple $\left(C_{T}, \lambda_{T}\right)$ of $\mathbf{R} \times \mathbf{R}_{+}^{*}$ such that $\max _{t \in[0, T]}|v(t, y)| \leq C_{T} e^{\lambda_{T} y^{2}}$ for all real $y$. Define; for every $a>\lambda_{T}$, the map $J_{v}: \mathbf{R}_{+} \times(0,1 / 4 a) \times \mathbf{R} \rightarrow \mathbf{R}$ by setting:

$$
J_{v}\left(t, u_{1}, u_{2}\right):=\int_{\mathbf{R}} v(t, x) \cdot \gamma\left(u_{1}, x-u_{2}\right) d x .
$$

Then $J_{v}$ is well defined. Moreover $\lim _{\left(t, u_{1}, u_{2}\right) \rightarrow\left(t_{0}, 0^{+}, l_{0}\right)} J_{v}\left(t, u_{1}, u_{2}\right)=v\left(t_{0}, l_{0}\right), \forall\left(t_{0}, l_{0}\right)$ in $[0, T] \times \mathbf{R}$.

It is easy to extend [Ben03b, Thm. 2.8] to the case of a Borel measure $m$ instead of the Lebesgue measure. The next result, which constitutes this extension, is more suitable that Thm. 2.6, when one deals with $L^{2}$-valued integrands. The proof being obvious, is then left to the reader.

Theorem 4.3. Let $m$ be a positive measure on $(\mathscr{R}, \mathcal{B}(\mathscr{R}))$ and $X: \mathscr{R} \rightarrow\left(L^{2}\right)$ be such that the function $t \mapsto S\left(X_{t}\right)(\eta)$ is measurable, for all $\eta$ in $\mathscr{S}(\mathbf{R})$, and such that $t \mapsto\left\|X_{t}\right\|_{0}$ belongs to $L^{1}(\mathscr{R}, m)$. Then $X$ is $(\mathcal{S})^{*}$-integrable over $\mathscr{R}$ and verifies:

$$
\left\|\int_{\mathscr{R}} X_{t} m(d t)\right\|_{0} \leq \int_{\mathscr{R}}\left\|X_{t}\right\|_{0} m(d t) .
$$

Through this subsection, we assume that $T>0$ and define $\mathscr{R}:=[0, T]$. We can now give the main result of this section. Denote $C^{1,2}([0, T] \times \mathbf{R}, \mathbf{R})$ the set of functions of two variables which belongs to $C^{1}([0, T], \mathbf{R})$ as function of their first variable and to $C^{2}(\mathbf{R}, \mathbf{R})$ as function of their second variable. The main result of this section is the following. 
Theorem 4.4. Let $T>0$. Let $f$ be a $C^{1,2}([0, T] \times \mathbf{R}, \mathbf{R})$ function. Furthermore, assume that there are constants $C \geq 0$ and $\lambda<\left(4 \max _{t \in[0, T]} R_{t}\right)^{-1}$ such that for all $(t, x)$ in $[0, T] \times \mathbf{R}$,

$$
\max _{t \in[0, T]}\left\{|f(t, x)|,\left|\frac{\partial f}{\partial t}(t, x)\right|,\left|\frac{\partial f}{\partial x}(t, x)\right|,\left|\frac{\partial^{2} f}{\partial x^{2}}(t, x)\right|\right\} \leq C e^{\lambda x^{2}} .
$$

Assume moreover that Assumption $(\mathscr{A})$ holds and that the map $t \mapsto R_{t}$ is both continuous and of bounded variations on $[0, T]$. Then the following equality holds in $\left(L^{2}\right)$ :

$$
f\left(T, G_{T}\right)=f(0,0)+\int_{0}^{T} \frac{\partial f}{\partial t}\left(t, G_{t}\right) d t+\int_{0}^{T} \frac{\partial f}{\partial x}\left(t, G_{t}\right) d^{\diamond} G_{t}+\frac{1}{2} \int_{0}^{T} \frac{\partial^{2} f}{\partial x^{2}}\left(t, G_{t}\right) d R_{t} .
$$

Proof. The general technique of proof of the Itô formula via the S-transform can be traced back to Kub83. The general structure of this proof is similar to the proof of Ben03b, Theorem 5.3]. However, one can not follow this latter completely since one does not assume (as it is the case for fBm) that $\mathcal{Z}_{R}^{T}=\{0\}$, where we set $\mathcal{Z}_{R}^{T}:=\mathcal{Z}_{R} \cap[0, T]$. Equality (4.5) may be rewritten as:

$$
\int_{0}^{T} \frac{\partial f}{\partial x}\left(t, G_{t}\right) d^{\diamond} G_{t}=f\left(T, G_{T}\right)-f(0,0)-\int_{0}^{T} \frac{\partial f}{\partial t}\left(t, G_{t}\right) d t-\frac{1}{2} \int_{0}^{T} \frac{\partial^{2} f}{\partial x^{2}}\left(t, G_{t}\right) d R_{t} .
$$

Thanks to (4.4) we may write, for every $K$ in $\left\{f, \frac{\partial f}{\partial t}, \frac{\partial f}{\partial x}, \frac{\partial^{2} f}{\partial x^{2}}\right\}$ and $t$ in $[0, T]$, that $\mathbf{E}\left[K\left(t, G_{t}\right)^{2}\right] \leq$ $M^{2}$, where we set $M^{2}:=C^{2}(1-4 \lambda \bar{R})^{-1 / 2}$ and $\bar{R}:=\sup \left\{R_{t} ; t \in[0, T]\right\}$. Moreover, $t \mapsto$ $\left\|K\left(t, G_{t}\right)\right\|_{0}$ belongs to $L^{1}([0, T], d t)$ while $t \mapsto\left\|\frac{\partial^{2} f}{\partial x^{2}}\left(t, G_{t}\right)\right\|_{0}$ belongs to $L^{1}\left([0, T], d R_{t}\right)$. The measurability of the maps $t \mapsto S\left(K\left(t, G_{t}\right)(\eta)\right.$ will become clear thanks to (4.7). A simple application of Theorem 4.3 then yields that all members on the right hand side of (4.6) exist and are in $\left(L^{2}\right)$. Moreover, Lemma 2.3 provides the upper-bound $\left|S\left(\frac{\partial f}{\partial x}\left(t, G_{t}\right) \diamond W_{t}^{(G)}\right)(\eta)\right| \leq M\left|g_{t}^{\prime}\right|_{-q} e^{|\eta|_{q}^{2}}$, for all $(\eta, t)$ in $\mathscr{S}(\mathbf{R}) \times[0, T]$, where $q$ is given by Assumption $\mathscr{A}_{(\mathrm{iii})}$. A straightforward application of Theorem 2.6 then shows that $\int_{0}^{T} \frac{\partial f}{\partial x}\left(t, G_{t}\right) d^{\diamond} G_{t}$ belongs to $(\mathcal{S})^{*}$. In order to prove the theorem, it then just remains to show that the $S$-transform of both sides of (4.6) are equal. For this purpose, we first give an integral representation of the S-transform of $K\left(t, G_{t}\right)$. Since $\mathbf{E}\left[: e^{<\cdot, \eta>}:\right]=1$, for every $\eta$ in $\mathscr{S}(\mathbf{R})$, one can define a probability measure $\mathbf{Q}_{\eta}$ on the space $(\Omega, \mathcal{F})$ by setting $\frac{d \mathbf{Q}_{\eta}}{d \mu} \stackrel{\text { def }}{=}: e^{<., \eta>}:$, where $\frac{d \mathbf{Q}_{\eta}}{d \mu}$ denotes the Radon-Nikodym derivative of $\mathbf{Q}_{\eta}$ with respect to $\mu$. To make computations easier we use the following obvious fact: $\mathcal{L}_{X+\mathscr{S}(X)(\eta)}^{\mu}=\mathcal{L}_{X}^{\mathrm{Q}_{\eta}}$, for every centered Gaussian random variable $X$ and $\eta$ in $\mathscr{S}(\mathbf{R})$, and where $\mathcal{L}_{Y}^{\rho}$ denotes the law of a random variable $Y$ under the probability measure $\rho$. In view of this fact, it is clear that $G_{t}$ is a Gaussian variable with mean $\left\langle g_{t}, \eta\right\rangle$ and variance $R_{t}$, under the probability measure $\mathbf{Q}_{\eta}$. One then gets, for every $t$ in $[0, T]$ and $\eta$ in $\mathscr{S}(\mathbf{R})$ :

$$
S\left(K\left(t, G_{t}\right)\right)(\eta)=\mathbf{E}_{\mathbf{Q}_{\eta}}\left[K\left(t, G_{t}\right)\right]=\int_{\mathbf{R}} K\left(t, u R_{t}^{1 / 2}+<g_{t}, \eta>\right) \frac{1}{\sqrt{2 \pi}} e^{-u^{2} / 2} d u .
$$

Denote $\Gamma_{R}:=[0, T] \backslash \mathcal{Z}_{R}^{T}$ and let $\eta$ be in $\mathscr{S}(\mathbf{R})$. In view of (4.7) we get:

$$
S\left(K\left(t, G_{t}\right)\right)(\eta)= \begin{cases}\int_{\mathbf{R}} K(t, v) \gamma\left(R_{t}, v-<g_{t}, \eta>\right) d v, & \forall t \in \Gamma_{R}, \\ K(t, 0), & \forall t \in \mathcal{Z}_{R}^{T} .\end{cases}
$$

Let $a$ be a real in $\left(\lambda,(4 \bar{R})^{-1}\right)$. Thanks to Lemma 4.2, we know that the map $J_{K}$ is well defined on $\Sigma_{a}:=[0, T] \times(0,1 / 4 a) \times \mathbf{R}$ and we clearly have:

$$
S\left(K\left(t, G_{t}\right)\right)(\eta)=J_{K}\left(t, R_{t},<g_{t}, \eta>\right), \forall t \in \Gamma_{R} .
$$

Moreover, it is clear that $J_{f}$ is a $C^{1}$-function on $\Sigma_{a}$. Denote, for every $\eta$ in $\mathscr{S}(\mathbf{R}), j_{\eta}:[0, T] \rightarrow \mathbf{R}$ the map defined by $j_{\eta}(t):=<g_{t}, \eta>$. According to Point 1 of Remark 1, $j_{\eta}$ is absolutely continuous on $[0, T]$. We first have the following result. 
Lemma 4.5. Let a be a real in $\left(\lambda,(4 \bar{R})^{-1}\right)$ and $J$ be the map, defined on $\Sigma_{a}$ by (4.3). For every $(t, \eta)$ in $\Gamma_{R} \times \mathscr{S}(\mathbf{R})$, one has the following equalities:

$\frac{J_{\partial^{2} f}}{\partial x^{2}}\left(t, R_{t}, j_{\eta}(t)\right)=2 \frac{\partial J_{f}}{\partial u_{1}}\left(t, R_{t}, j_{\eta}(t)\right) ; J_{\frac{\partial f}{\partial x}}\left(t, R_{t}, j_{\eta}(t)\right)=\frac{\partial J_{f}}{\partial u_{2}}\left(t, R_{t}, j_{\eta}(t)\right) J_{\frac{\partial f}{\partial t}}\left(t, R_{t}, j_{\eta}(t)\right)=\frac{\partial J_{f}}{\partial t}\left(t, R_{t}, j_{\eta}(t)\right)$.

Proof of Lemma 4.5. Using from one hand the equality $\frac{\partial \gamma}{\partial t}=\frac{1}{2} \frac{\partial^{2} \gamma}{\partial x^{2}}$, valid on $\mathbf{R}_{+}^{*} \times \mathbf{R}$, and, form the other hand the theorem of differentiation under the integral sign, in a neighborhood of every $\left(t, u_{1}, u_{2}\right)$ in $\Sigma_{a}$, provide equalities stated in Lemma 4.5] on each $\left(t, u_{1}, u_{2}\right)$ of $\Sigma_{a}$ and then allows us to conclude.

Using Lemma 4.5, one gets, for every $\eta$ in $\mathscr{S}(\mathbf{R})$,

$$
\begin{aligned}
\mathcal{I}_{\eta}^{(1)} & :=\int_{0}^{T} S\left(\frac{\partial^{2} f}{\partial x^{2}}\left(t, G_{t}\right)\right)(\eta) d R_{t}=\int_{\Gamma_{R}} J_{\partial^{2} f}\left(t, R_{t}, j_{\eta}(t)\right) d R_{t}=2 \int_{\Gamma_{R}} \frac{\partial J_{f}}{\partial u_{1}}\left(t, R_{t}, j_{\eta}(t)\right) d R_{t} \\
\mathcal{I}_{\eta}^{(2)} & :=\int_{0}^{T} S\left(\frac{\partial f}{\partial x}\left(t, G_{t}\right)\right)(\eta) S\left(W_{t}^{(G)}\right)(\eta) d t=\int_{\Gamma_{R}} \frac{\partial J_{f}}{\partial u_{2}}\left(t, R_{t}, j_{\eta}(t)\right) d j_{\eta}(t) \\
\mathcal{I}_{\eta}^{(3)} & :=\int_{0}^{T} S\left(\frac{\partial f}{\partial t}\left(t, G_{t}\right)\right)(\eta) d t=\int_{\Gamma_{R}} \frac{\partial J_{f}}{\partial t}\left(t, R_{t}, j_{\eta}(t)\right) d t+\int_{\mathcal{Z}_{R}^{T}} \frac{\partial f}{\partial t}(t, 0) d t
\end{aligned}
$$

Thus, in order to end the proof, one just has to establish the following equality:

$$
\begin{aligned}
S\left(f\left(T, G_{T}\right)\right)(\eta)-S(f(0,0))(\eta)=\int_{\Gamma_{R}} & \frac{\partial J_{f}}{\partial t}\left(t, R_{t}, j_{\eta}(t)\right) d t+\int_{\Gamma_{R}} \frac{\partial J_{f}}{\partial u_{1}}\left(t, R_{t}, j_{\eta}(t)\right) d R_{t} \\
& +\int_{\Gamma_{R}} \frac{\partial J_{f}}{\partial u_{2}}\left(t, R_{t}, j_{\eta}(t)\right) d j_{\eta}(t)+\int_{\mathcal{Z}_{R}^{T}} \frac{\partial f}{\partial t}(t, 0) d t
\end{aligned}
$$

Since $\Gamma_{R}$ is an open set of $[0, T]$, that does not contain 0 , it can be written under the form

$$
\Gamma_{R}=\bigsqcup_{i \in \mathbf{N}}\left(a_{i}, b_{i}\right) \sqcup(b, T],
$$

where all the intervals in (4.15) are disjoint and where, by convention, $(x, y)=(x, y]=\emptyset$, for every reals $x$ and $y$ such that $x \geq y$. Note moreover that every element of $\left\{a_{i}, b_{i}, i \in \mathbf{N}\right\}$ (as well as $b$, if $(b, T] \neq \emptyset)$ belongs to $\mathcal{Z}_{R}^{T}$. We need to distinguish between two cases:

First case: $\exists\left(a^{\prime}, b^{\prime}\right) \in(0, T)^{2}$ with $a^{\prime}<b^{\prime}$ s.t. $\left(0, a^{\prime}\right)$ and $\left(b^{\prime}, T\right]$ are both subsets of $\Gamma_{R}$.

Define $a:=\sup \left\{a^{\prime} \in[0, T]\right.$, s.t. $\left.\left(0, a^{\prime}\right) \subset \Gamma_{R}\right\}$ and $b:=\inf \left\{b^{\prime} \in[0, T]\right.$, s.t. $\left.\left(b^{\prime}, T\right] \subset \Gamma_{R}\right\}$. Even if one has to consider a subset $I$ of $\mathbf{N}$, one may assume, and we will in the sequel, that $\left(a_{i}, b_{i}\right) \neq \emptyset$, for every $i$ in $\mathbf{N}$. One can find $\rho$ in $\mathbf{R}_{+}^{*}$ such that $(\rho, a-\rho) \neq \emptyset,(b+\rho, T-\rho) \neq \emptyset$. Moreover, for every $i$ in $\mathbf{N}$, one can find $\rho_{i}$ in $\mathbf{R}_{+}^{*}$ such that $\left(a_{i}+\rho_{i}, b_{i}-\rho_{i}\right) \neq \emptyset$. Since all these intervals belong to $\Gamma_{R}$, one can apply Lemma 4.1, with $L=J_{f}$, on each one of them. We then get, for every interval $(x, y)$ in the set of intervals $\Upsilon:=\left\{(\rho, a-\rho),(b+\rho, T-\rho),\left(a_{i}+\rho_{i}, b_{i}-\rho_{i}\right) ; i \in \mathbf{N}\right\}$,

$$
\begin{aligned}
& J_{f}\left(y, R_{y}, j_{\eta}(y)\right)-J_{f}\left(x, R_{x}, j_{\eta}(x)\right) \\
& \quad=\int_{x}^{y} \frac{\partial J_{f}}{\partial t}\left(t, R_{t}, j_{\eta}(t)\right) d t+\int_{x}^{y} \frac{\partial J_{f}}{\partial u_{1}}\left(t, R_{t}, j_{\eta}(t)\right) d R_{t}+\int_{x}^{y} \frac{\partial J_{f}}{\partial u_{2}}\left(t, R_{t}, j_{\eta}(t)\right) d j_{\eta}(t) .
\end{aligned}
$$

For any interval $\left(x^{\prime}, y^{\prime}\right)$ which belongs to $\left\{(0, a),(b, T),\left(a_{i}, b_{i}\right) ; i \in \mathbf{N}\right\}$, there exists a sequence of elements $\left(x_{n}, y_{n}\right)_{n \in \mathbf{N}}$ in $\Upsilon^{\mathbf{N}}$ such that $\left(x_{n}, y_{n}\right) \rightarrow\left(x^{\prime}, y^{\prime}\right)$, as $n \rightarrow+\infty$ and such that $\left[x_{n}, y_{n}\right] \subset\left(x^{\prime}, y^{\prime}\right)$. Lemma 4.2 then provides the convergence of the left hand side of $\left(\Lambda_{x_{n}, y_{n}}\right)$ to $f\left(y^{\prime}, 0\right)-f\left(x^{\prime}, 0\right)$, if $\left(x^{\prime}, y^{\prime}\right)$ belongs to $\left\{(0, a),\left(a_{i}, b_{i}\right) ; i \in \mathbf{N}\right\}$, and to $S\left(f\left(T, G_{T}\right)\right)(\eta)-f(b, 0)$, 
if $\left(x^{\prime}, y^{\prime}\right)=(b, T)$. Besides, the Lebesgue's dominated convergence theorem applies to each integrand of the right hand side of $\left(\Lambda_{x_{n}, y_{n}}\right)$, since they are all continuous. This provides the convergence of the right hand side of $\left(\Lambda_{x_{n}, y_{n}}\right)$ to $\int_{x^{\prime}}^{y^{\prime}} \frac{\partial J_{f}}{\partial t}\left(t, R_{t}, j_{\eta}(t)\right) d t+\int_{x^{\prime}}^{y^{\prime}} \frac{\partial J_{f}}{\partial u_{1}}\left(t, R_{t}, j_{\eta}(t)\right) d R_{t}+$ $\int_{x^{\prime}}^{y^{\prime}} \frac{\partial J_{f}}{\partial u_{2}}\left(t, R_{t}, j_{\eta}(t)\right) d j_{\eta}(t)$, for any $\left(x^{\prime}, y^{\prime}\right)$ in $\left\{(0, a),(b, T),\left(a_{i}, b_{i}\right) ; i \in \mathbf{N}\right\}$. In view of (4.9) and (4.10), and making the summation of $\left(\Lambda_{a_{i}, b_{i}}\right)$, over all $i \in \mathbf{N}$, we then get:

$$
\begin{aligned}
& S\left(f\left(T, G_{T}\right)\right)(\eta)-S(f(0,0))(\eta)-\left(f(b, 0)-f(a, 0)-\sum_{i \in \mathbf{N}}\left(f\left(b_{i}, 0\right)-f\left(a_{i}, 0\right)\right)\right) \\
& \quad=\int_{\Gamma_{R}} \frac{\partial J_{f}}{\partial t}\left(t, R_{t}, j_{\eta}(t)\right) d t+\int_{\Gamma_{R}} \frac{\partial J_{f}}{\partial u_{1}}\left(t, R_{t}, j_{\eta}(t)\right) d R_{t}+\int_{\Gamma_{R}} \frac{\partial J_{f}}{\partial u_{2}}\left(t, R_{t}, j_{\eta}(t)\right) d j_{\eta}(t) .
\end{aligned}
$$

Denote $\Delta:=\int_{\mathcal{Z}_{R}^{T}} \frac{\partial f}{\partial t}(t, 0) d t$, one has the equality:

$$
\begin{aligned}
\Delta & =\int_{[0, T]} \frac{\partial f}{\partial t}(t, 0) d t-\int_{\Gamma_{R}} \frac{\partial f}{\partial t}(t, 0) d t=\int_{[0, T] \backslash(0, a] \cup(b, T]} \frac{\partial f}{\partial t}(t, 0) d t-\sum_{i \in \mathbf{N}} \int_{a_{i}}^{b_{i}} \frac{\partial f}{\partial t}(t, 0) d t \\
& =f(b, 0)-f(a, 0)-\sum_{i \in \mathbf{N}}\left(f\left(b_{i}, 0\right)-f\left(a_{i}, 0\right)\right) .
\end{aligned}
$$

Using (4.17), Equality (4.16) then reads:

$$
\begin{aligned}
& S\left(f\left(T, G_{T}\right)\right)(\eta)-S(f(0,0))(\eta)-\int_{\mathcal{Z}_{R}^{T}} \frac{\partial f}{\partial t}(t, 0) d t \\
& \quad=\int_{\Gamma_{R}} \frac{\partial J_{f}}{\partial t}\left(t, R_{t}, j_{\eta}(t)\right) d t+\int_{\Gamma_{R}} \frac{\partial J_{f}}{\partial u_{1}}\left(t, R_{t}, j_{\eta}(t)\right) d R_{t}+\int_{\Gamma_{R}} \frac{\partial J_{f}}{\partial u_{2}}\left(t, R_{t}, j_{\eta}(t)\right) d j_{\eta}(t),
\end{aligned}
$$

which is nothing but (4.14) and therfore ends the proof in this case.

Second case: There is no $\left(a^{\prime}, b^{\prime}\right)$ in $(0, T)^{2}$ with $a^{\prime}<b^{\prime}$ s.t. both $\left(0, a^{\prime}\right) \&\left(b^{\prime}, T\right]$ are subsets of $\Gamma_{R}$.

Since the cases of 0 and $T$ can be treated in the same manner, we only treat here the case of $T$. We then assume that there is no $b^{\prime}$ in $(0, T)$ such that $\left(b^{\prime}, T\right] \subset \Gamma_{R}$. We need to distinguish between two cases. If there exists $b^{\prime}$ in $(0, T)$ such that $\left(b^{\prime}, T\right] \subset \mathcal{Z}_{R}^{T}$ then the problem can be reduced to establish (4.5) between 0 and $\hat{b}$, where $\hat{b}:=\inf \left\{b \in(0, T) ;\left[b^{\prime}, T\right] \subset \mathcal{Z}_{R}^{T}\right\}$. Otherwise, one can find an increasing sequence $\left(T_{n}\right)_{n \in \mathbf{N}}$ of $\Gamma_{R}^{\mathbf{N}}$, which converge to $T$. For every $n$ in $\mathbf{N}$, denote $\left(a_{i}^{(n)}, b_{i}^{(n)}\right)$ the interval $\left(a_{i}, b_{i}\right)$ of $\Gamma_{R}$ which contains $T_{n}$. For every integer $n,\left(a_{i}^{(n)}, T_{n}\right]$ is a non empty subset of $\Gamma_{R}$. Therefore, one can use first case to establish (4.5) between 0 and $T_{n}$. To establish the equality of S-transform of both sides of (4.5), (between 0 and $T_{n}$ ), it then remains to apply, from one hand Lemma 4.2 to $J_{f}\left(T_{n}, R_{T_{n}}, j_{\eta}\left(T_{n}\right)\right)$ and, form the other hand, Lebesgue's dominated convergence theorem to the following integrals:

$$
\begin{aligned}
& \mathcal{I}_{\eta, n}^{(1)}:=\int_{0}^{T_{n}} S\left(\frac{\partial^{2} f}{\partial x^{2}}\left(t, G_{t}\right)\right)(\eta) d R_{t}=2 \int_{\Gamma_{R}^{(n)}} \frac{\partial J_{f}}{\partial u_{1}}\left(t, R_{t}, j_{\eta}(t)\right) d R_{t}, \\
& \mathcal{I}_{\eta, n}^{(2)}:=\int_{0}^{T_{n}} S\left(\frac{\partial f}{\partial x}\left(t, G_{t}\right)\right)(\eta) S\left(W_{t}^{(G)}\right)(\eta) d t=\int_{\Gamma_{R}^{(n)}} \frac{\partial J_{f}}{\partial u_{2}}\left(t, R_{t}, j_{\eta}(t)\right) d j_{\eta}(t), \\
& \mathcal{I}_{\eta, n}^{(3)}:=\int_{0}^{T_{n}} S\left(\frac{\partial f}{\partial t}\left(t, G_{t}\right)\right)(\eta) d t=\int_{\Gamma_{R}^{(n)}} \frac{\partial J_{f}}{\partial t}\left(t, R_{t}, j_{\eta}(t)\right) d t+\int_{\mathcal{Z}_{R}^{T,(n)}} \frac{\partial f}{\partial t}(t, 0) d t,
\end{aligned}
$$

where $\Gamma_{R}^{(n)}$ denotes $\Gamma_{R} \cap\left[0, T_{n}\right]$ and $\mathcal{Z}_{R}^{T,(n)}$ denotes $\mathcal{Z}_{R}^{T} \cap\left[0, T_{n}\right]$. This result and the fact that $J_{f}$ is a $C^{1}$ function on $\Sigma_{a}$ allows us to apply [Kuo96, Theorem 8.6] and thus to conclude. 


\subsection{Comparison with other Itô formulas for Gaussian processes}

Since [AMN01], many Itô formula for Gaussian processes have been established. If one excepts Itô formula for Gaussian semimartingales, that are well known, all the Itô formulas provided, for Gaussian processes in general, in the literature of functional extensions so far, namely: [AMN01, Theorems 1 \& 2], [MV05, Theorem 31], [NT06, Theorem 1], [KRT07, Corollary 8.13], [KR10, Proposition 11.7] and [LN12, Theorem 3.2], are established using the divergence type integral. A requirement of all these previous references is that the variance function $t \mapsto R_{t}$ is, at least, continuous and with bounded variations on $[0, T]$. Assuming the continuity of $R$ seems reasonable. Indeed, otherwise, as the anonymous referee noticed, Equality (4.5) may fail for very simple functions $f$, such as $f(x):=x^{4}$. If one excepts [KRT07, Corollary 8.13], another requirements of theses references above is that the function $f$ is of class $C^{2}$ and, together with all its derivatives, with sub-exponential growth (i.e. fulfills (4.4)). In view of this fact, it appears that the assumptions made in Theorem 4.4 are minimal. However, to see to what extent Theorem 4.4 generalizes Itô formulas for Gaussian processes that already exist, let us make a detailed comparison.

\section{Comparison with the conditions on function $f$}

The function $f$ (i.e. $f(t, x):=f(x))$ is assumed to be of class $C^{\infty}$ in [MV05, Theorem 31] and in [KR10, Proposition 11.7], and of class $C^{7}$ in[NT06, Theorem 1]. In [KRT07, Corollary 8.13] and $f$ is assumed to be of class $C^{2}$ but not with sub-exponential growth; the second derivative of $f$ therein is assumed to be bounded. However, since the stochastic calculus for Gaussian processes, developed in [KRT07] requires that the covariance function has a planar bounded variation, which corresponds to "regular" processes (such as fBm for $H>1 / 2$ ), one easily sees that the price to pay for relaxing the assumption on the growth of $f$ is that one can not deal with irregular Gaussian processes (that is precisely to overcome this limitation on the regularity of $G$ that [KR10] has been written. However, as we stated above, this latter reference requires much more than the growth condition we make on $f$ ).

\section{Comparison with the assumptions made on $G$}

- Comparison with the assumptions made on $R$

The variance function $t \mapsto R_{t}$ is assumed to: be continuous and of bounded variations on $[0, T]$ in [KRT07, Corollary 8.13], be of class $C^{2}$ on $\mathbf{R}_{+}^{*}$ in [MV05, Theorem 31], fulfill Assumptions (3) et (4) in [NT06, Theorem 1], fulfill Assumptions (A), (B) and (C) in [KR10, Proposition 11.7] and, in [LN12, Theorem 3.2], to verify the two following conditions:

(i) for every $s$ in $[0, T]$, the map $t \mapsto R(t, s)$ is absolutely continuous on $[0, T]$;

(ii) there exists $\alpha>1$ such that: $\sup _{t \in[0, T]} \int_{0}^{T}\left|\frac{\partial R}{\partial s}(s, t)\right|^{\alpha} d s<+\infty$.

- Other assumptions

In [AMN01, Theorems $1 \& 2$ ], in addition to the assumptions made in Theorem 4.4, the kernel $K$ has to fulfill Assumptions (K1) to (K3), in the singular case, and (K1) to (K4), in the regular case. Other assumptions on the process $G$ are difficult to compare in general. As it is stated in Remark 1, a key propoerty in our construction of integral is that the maps $t \mapsto$ $\mathbf{E}\left[G_{t} \int_{\mathbf{R}} \eta(s) d B_{s}\right]$ are absolutely continuous w.r.t. the Lebesgue measure for every $\eta$ in $\mathscr{S}(\mathbf{R})$. A related assumption in other papers is that the functions $t \mapsto \mathbf{E}\left[G_{t} G_{s}\right]$ or $t \mapsto \mathbf{E}\left[G_{t} B_{s}\right]$ are absolutely continuous or of bounded variations for every $s$, see [LN12, AMN01]. However These assumptions do not, in general, imply each other. In an another class of papers, namely [KRT07, some quadratic variation type conditions are imposed on $G$, e.g. in [KRT07] or in [NT06]. These assumptions are even more difficult to compare with the present setting and would lead us too 
far from the goal of this present work. We therefore postpone a more detailed comparison to a future work.

Note also that he Itô formula provided in [LN12] is extended in this work, while the other results presented in [LN12] are extended in [Leb17].

In view of the arguments developed above, it appears that the Itô formula we present here offers improvements on the ones presented in [AMN01, CN05, MV05, NT06, KR10, LN12], by allowing one to have less restrictive hypotheses. Of course all the Gaussian processes in $\mathscr{G}$ of "reference" fulfill assumptions of Theorem 4.4. In the case of $\mathrm{mBm}$ one needs to assume that $h$ is a $C^{1}$ function with its derivative bounded on $\mathscr{R}_{D}$. Note moreover that, applying Theorem 4.4 when $G$ is a fBm (resp. a mBm) allows one to recover [Ben03a, Theorem 4.1 \& Rk. 4.6] (resp. [LLV14, Theorem 5.5]). When $G$ is a $\mathscr{V}_{\gamma}$ - process one recovers and extends, as we showed above, [MV05, Theorem 31].

\section{Comparison with other stochastic integrals}

\section{Forewords}

In order to define the divergence integral of a continuous Gaussian process $G$ in the way of AMN01 and then of MV05, it is essential to first know a representation of $G$ on a compact set of the form $[0, T]$. In general, [HH93, Theorem 4.1] ensures that any Gaussian process may be written as a sum of two terms; one of them being $\sum_{i=1}^{N} \int_{0}^{t} K_{i}(t, u) d W_{i}(u)$, where $N$ is a positive integer (possibly infinite) and $W$ is a Brownian motion. However it is not an easy task to obtain such a decomposition for a given process $G$. For instance, although a kernel is known for $\mathrm{fBm}$, this is not the case of bifractional motion HV03. Likewise, writing the moving average and harmonizable multifractional Brownian motion under this form remains an open problem (see [LLVH14, Section 5] for more details). Moreover, Gaussian bridges in general are an example of Gaussian processes which do not admit "proper" Volterra representation, i.e. that can not be written under the form (1.2) (see [SV14, Ex. 3.3]). Thus, there is no hope to use [AMN01] nor [MV05] in order to build a stochastic integral wrt Gaussian processes of the form (1.1), for which one does not know any integral representation on a compact set included in $[0, T]$. To overcome this deficiency one then might consider [NT06, KRT07, KR10, LN12, SV14]. As we stated above, in these latter references one needs that the covariance function fulfills some requirements. However, it happens sometimes that one has to deal with Gaussian processes, given under the form (1.1), for which one does not know how to compute the covariance function, such as the one where $g_{t}$ is defined by setting $g_{t}(u)=\mathbf{1}_{[0, t]}(u) K_{h(t)}(t, u)$, where $h:[0, T] \rightarrow(0,1)$ is a continuous deterministic function and where the family of Kernel $\left(K_{H}\right)_{H \in(0,1)}$ is the one defined in [Nua06, (5.8)] in the case where $H \in(1 / 2,1)$ and in [Nua06. Proposition 5.1.3] in the case where $H \in(0,1 / 2)$. As a consequence our stochastic calculus it is the only one available when the Gaussian process $G$ can be written under the form (1.1) but not under any of the form (1.2) nor (1.3); or when the stochastic calculus provided in [KRT07, KR10, LN12] does not apply. The work provided in [KRT07] offers an alternative to the previous methods to build a stochastic integral wrt continuous Gaussian processes, for which one knows the covariance function. Introducing the concept of covariance measure structure, the authors built and developed a stochastic calculus wrt "regular" processes (such as fBm with $H \geq 1 / 2$ ). This work has been extended to the "singular" case in [KR10]. This approach is particularly suitable when the kernel is not explicitly known, under any of the representations (1.1) to (1.3), (like in the case of bifractional motion [HV03]). However, the Itô formula in [KR10] is quite restrictive. The conditions required in [KRT07] are not so restrictive but they do not allow one to deal with "irregular" Gaussian processes, by the very essence of covariance measure structure.

In this section we make first, in Subsection [5.1, a comparison of the Wick-Itô stochastic integral 
we developed above with the functional extensions of stochastic integrals developed in AMN01, MV05 and then, in Subection 5.2, with the Itô integral.

\subsection{Comparison with Malliavin Calculus or divergence type integrals}

We start by making the comparison between our Wick-Itô integral and the divergence type integral developed in [AMN01]. We will then show, in Section 5.1.2, that the Wick-Itô integral fully generalizes the (extended) Skorohod integral developed in MV05. Let $T>0$ be fixed and let us take $\mathscr{R}=[0, T]$. Let $G:=\left(G_{t}\right)_{t \in[0, T]}$ be a Volterra process.

\subsubsection{Comparison with divergence type integral of [AMN01]}

The goal of this section is to compare the Wick-Itô integral wrt $G$ to the divergence integral wrt $G$, defined in [AMN01] and in [MV05] and studied in [AMN01, Nua05] and in [MV05]. In AMN01 $G$ is a assumed to be a continuous process while it is not assumed to be continuous in MV05. One therefore will assume (in Subsection 5.1.1 only) that $G$ is continuous on $[0, T]$. $G$ being a Volterra process, it can be written, for any real $t$ in $[0, T]$,

$$
G_{t}=\int_{0}^{t} K(t, s) d W_{s}
$$

where the kernel $K(t, s)$, defined on $[0, T]^{2}$, is such that $K(t, s)=0$ on the set $[0, T]^{2} \backslash\{(u, v) \in$ $(0, T] \times[0, T]: v<u\}$ and verifies for any $t \geq 0, \widehat{K}_{t}:=\int_{0}^{t} K(t, s)^{2} d s<\infty$.

Denote $L^{2}\left(\Omega, L^{2}([0, T])\right)$ the set of random process $u$ such that $\|u\|_{L^{2}\left(\Omega, L^{2}([0, T])\right)}^{2}:=\mathbf{E}\left[\int_{0}^{T} u_{t}^{2} d t\right]<$ $+\infty$. The main result of this section is Theorem [5.3, which states that every process $u$ which belongs to $L^{2}\left(\Omega, L^{2}([0, T])\right)$ and that belongs to the domain of the divergence of $G$ is also Wick-Itô integrable wrt $G$, on $[0, T]$. Moreover, one has the equality $\int_{0}^{T} u_{s} \delta G_{s}=\int_{0}^{T} u_{s} d G_{s}$, where $\int_{0}^{T} u_{s} \delta G_{s}$ denotes the divergence integral on $[0, T]$, associated to $G$, that has been defined in AMN01. In order to state rigorously this result we briefly recall some elements and notations of stochastic calculus of variations wrt $G$ (for a presentation of Malliavin calculus, see e.g. [Bal03, Nua06]), as well as the approach of AMN01 and Nua05 for the construction of a stochastic integral wrt to Volterra processes. The real $T>0$ being fixed, one still note $G$ the process $\left(G_{t}\right)_{t \in[0, T]}$ since there is no risk of confusion. $G$ being a centered Gaussian process, denote $\mathcal{H}_{T}$ the reproducing kernel Hilbert space (R.K.H.S.) defined as the closure of the set $\mathcal{E}_{T}:=\operatorname{span}\left\{\mathbf{1}_{[0, t]}, t \in[0, T]\right\}$, with respect to the inner product $<,>_{\mathcal{H}_{T}}$, that has been defined by setting $\left\langle\mathbf{1}_{[0, t]}, \mathbf{1}_{[0, s]}\right\rangle_{\mathcal{H}_{T}}:=R_{t, s}$. Denote $H_{1}$ the first Wiener chaos of $G$ and $G(\varphi)$ the image in $H_{1}$ of an element $\varphi$ of $\mathcal{H}_{T}$ by the isometry, between $\mathcal{H}_{T}$ and $H_{1}$, that associates $\mathbf{1}_{[0, t]}$ to $G_{t}$.

Remark 8. It is not always true that the bilinear form $<,>_{\mathcal{H}_{T}}$ defined by $\left\langle\mathbf{1}_{[0, t]}, \mathbf{1}_{[0, s]}\right\rangle_{\mathcal{H}_{T}}:=$ $R_{t, s}$ is an inner product. For example, for the Brownian bridge $\widehat{B}:=\left(\widehat{B}_{t}\right)_{t \in[0,1]}$ on $[0,1]$, one has $\left\|\mathbf{1}_{[0,1]}\right\|_{\mathcal{H}_{T}}=0$. For this reason we will assume in the sequel that $<,>_{\mathcal{H}_{T}}$, defined above, is really an inner product. The reader interested in details on Reproducing Kernels Hilbert Spaces may refer to [Jan97, Chap.8] as well as to [LLV14, Appendix B] in the case of mBm.

Define $\mathcal{S}:=\left\{V=f\left(G\left(\varphi_{1}\right), G\left(\varphi_{2}\right), \ldots, G\left(\varphi_{n}\right)\right), f \in C_{b}^{\infty}\left(\mathbf{R}^{n}\right), \varphi_{i} \in \mathcal{H}_{T}, i=1, \ldots, n\right\}$. For an element $V$ of $\mathcal{S}$, one defines the derivative operator $D^{G}$ as:

$$
D^{G} V:=\sum_{i=1}^{n} \frac{\partial f}{\partial x_{i}}\left(G\left(\varphi_{1}\right), G\left(\varphi_{2}\right), \ldots, G\left(\varphi_{n}\right)\right) \varphi_{n}
$$

The derivative operator $D^{G}$ is a closable unbounded operator from $L^{2}(\Omega)$ into $L^{2}\left(\Omega ; \mathcal{H}_{T}\right)$. We note $\mathbb{D}_{G}$ the closure of $\mathcal{S}$ with respect to the norm defined by $\|V\|_{G, 1,2}:=\left(\mathbf{E}\left[V^{2}\right]+\right.$ $\left.\mathbf{E}\left[\left\|D^{G} V\right\|_{L^{2}\left(\Omega ; \mathcal{H}_{T}\right)}^{2}\right]\right)^{\frac{1}{2}}$. We denote by $\delta_{G}$, and call divergence integral with respect to $G$, the 
adjoint of the derivative operator $D^{G}$. The domain of $\delta^{G}$ in $L^{2}$, denoted $\mathbb{D o m}\left(\delta^{G}\right)$, is the set of the elements $u$ in $L^{2}\left(\Omega ; \mathcal{H}_{T}\right)$ such that there exists a constant $c$ verifying, for all $V$ in $\mathcal{S},\left|\mathbf{E}\left(<D^{G} V, u>_{\mathcal{H}_{T}}\right)\right| \leq c\|V\|_{2}$, where \|\|$_{2}$ denotes the norm in $L^{2}(\Omega)$. If $u$ belongs to $\operatorname{Dom}\left(\delta^{G}\right), \delta^{G}(u)$ is the element of $L^{2}(\Omega)$ defined by the duality relationship: $\mathbf{E}\left(V \delta^{G}(u)\right)=$ $\mathbf{E}\left(<D^{G} V, u>_{\mathcal{H}_{T}}\right)$, for all $V$ in $\mathbb{D}_{G}$. We will simply denote, in the sequel, $D, \mathbb{D}, \delta$ and \|\|$_{1,2}$ when $G$ is a Brownian motion. Define now the linear operator $K_{-}: \mathcal{E}_{T} \rightarrow L^{2}([0, T])$ by $K_{-}\left(\mathbf{1}_{[0, t]}\right):=K(t,$.$) and denote \|\|_{\mathcal{H}_{T}}$ the norm on $\mathcal{H}_{T}$ which derives from the inner product $<,>_{\mathcal{H}_{T}}$. Since $\|\varphi\|_{\mathcal{H}_{T}}=\left\|K_{-}(\varphi)\right\|_{L^{2}([0, T])}$, for every $\varphi$ in $\mathcal{E}_{T}$, it is clear that the operator $K_{-}$can be extended to a linear isometry, still denoted $K_{-}$, between $\left(\mathcal{H}_{T},\|\|_{\mathcal{H}_{T}}\right)$ and a closed subset of $L^{2}([0, T])$. Besides, one can show, AMN01, (12)], that $\mathbb{D o m}\left(\delta_{G}\right)=\left(K_{-}\right)^{-1}(\mathbb{D o m}(\delta))$. Moreover, for a process $v$ in $\mathbb{D o m}\left(\delta_{G}\right)$ one has:

$$
\delta_{G}(v)=\int_{0}^{T}\left(K_{-} v\right)(s) \delta W_{s} .
$$

In other words, $\delta_{G}(v)$, the divergence integral of $v$ wrt $G$, also noted $\int_{0}^{T} v(s) \delta G_{s}$, verifies the equality $\int_{0}^{T} v(s) \delta G_{s}=\int_{0}^{T}\left(K_{-} v\right)(s) \delta W_{s}$. In order to prove Theorem 5.3 below, one needs to define the adjoint of the operator $K_{-}$, that we will denote $K_{+}$, not only on the set $\mathcal{E}_{T}$ but also on $\mathscr{S}(\mathbf{R})$. For this reason we recall the two following hypotheses, given in [Nua05] for fBm, that we will make in the sequel on the kernel $K(t, s)$.

H1) $K(t, s)$ is continuously differentiable on $\{0<s<t \leq T\}$ and its partial derivative verify the following integrability condition:

$$
\sup _{\varepsilon \leq t \leq T} \int_{t}^{T}\left|\frac{\partial K}{\partial r}(r, t)\right|(r-t) d r+\int_{0}^{t}\left|\frac{\partial K}{\partial t}(t, s)\right|(t-s) d s<\infty
$$

for any $\varepsilon$ in $(0, T)$. Moreover, $t \mapsto \int_{0}^{t \wedge b} \frac{\partial K}{\partial t}(t, s)(t \wedge b-s \vee a)_{+} d s$ is continuous on $(0, T]$, for all $0 \leq a \leq b$.

H2) The function $k(t):=\int_{0}^{t} K(t, s) d s$ is continuously differentiable on $(0, T]$.

We present here the arguments given in [Nua05, Section 2] for fBm about the operator $K_{+}$, but in a slightly different manner. Denote $C_{b}^{1}(\mathbf{R})$ the set of differentiable functions which are bounded together with its derivatives. Hypotheses H1) and H2) allow us to define the operator $K_{+}$on $\mathcal{E}_{T} \cup C_{b}^{1}(\mathbf{R})$ by setting, for every $t$ in $[0, T],\left(K_{+} \varphi\right)(t):=k^{\prime}(t) \varphi(t)+\int_{0}^{t} \frac{\partial K}{\partial t}(t, r)(\varphi(r)-\varphi(t)) d r$. In view of [Nua05, p.116], it is easy to check that we have, for any $(\psi, \varphi)$ in $\mathcal{E}_{T} \times \mathcal{E}_{T}$, the equality

$$
<K_{+}(\varphi), \psi>_{L^{2}([0, T])}=<\varphi, K_{-}(\psi)>_{L^{2}([0, T])} .
$$

It is clear that one has, in this section, $g_{t}:=K_{-}\left(\mathbf{1}_{[0, t]}\right)$, for every $t$ in $[0, T]$. It is established in [Nua05, Propostion 2] that $g_{t}^{\prime}$ exists and that $g_{t}^{\prime}=K_{+}().(t)$ for every $t$ in $(0, T]$. However it is not possible to establish that $t \mapsto W_{t}^{(G)}$ is $\left(\mathcal{S}^{*}\right)$-integrable on $[0, T]$ without any additional assumption. Moreover one needs to be able to establish that $\int_{0}^{T} u_{s} d G_{s}$ exist for a reasonable class of processes $u$. Thus, following [Nua05, Proposition 7], we will assume in the sequel the following condition:

H3) The function $C: t \mapsto\left|k^{\prime}(t)\right|+\int_{0}^{t}\left|\frac{\partial K}{\partial t}(t, r)\right|(t-r) d r$ belongs to $L^{2}([0, T])$.

Remark 9. It is clear that $\left.\left.H_{1}\right), H_{2}\right)$ and $\left.H_{3}\right)$ entail that Assumptions $\mathscr{D}_{(i)}$ and $\mathscr{D}_{(i i)}$ hold. We will show, in the next subsection (Remark 111), that they are not always necessary.

The following result will be useful in the proof of Theorem 5.3 below. 
Lemma 5.1. If Assumptions $\left.\left.H_{1}\right), H_{2}\right)$ and $\left.H_{3}\right)$ hold, any process $u$ in $L^{2}\left(\Omega, L^{2}([0, T])\right)$ is Wick-Itô integrable with respect to $G$.

Proof. The proof of this lemma, which consists on verifying that Condition $(\underline{\mathcal{I}})$ is verified with $p=q=2$, can be found in [Nua05, Proposition 7].

Since one has: $\left|\left(K_{+} \eta\right)(t)\right| \leq\left|k^{\prime}(t)\right|\|\eta\|_{\infty}+\left\|\eta^{\prime}\right\|_{\infty} \int_{0}^{t}\left|\frac{\partial K}{\partial t}(t, r)\right|(t-r) d r$, for every $(\eta, t)$ in $\mathscr{S}(\mathbf{R}) \times$ $[0, T]$, Hypothesis H3) implies in particular that $K_{+}(\eta)$ belongs to $L^{2}([0, T])$. Note moreover that, for every $\eta$ in $\mathscr{S}(\mathbf{R})$,

$$
K_{+}(\eta)(t)=<\delta_{t}, K_{+}(\eta)>=\frac{d}{d t}<K_{-}\left(\mathbf{1}_{[0, t]}\right), \eta>_{L^{2}([0, T])} .
$$

The following result, which is a consequence of results given in [Nua05, Section 2 and Proposition 7], will be essential in order to prove Theorem 5.3 below. Denote $\overline{\mathcal{E}}_{T}\|\|_{T}$ the closure of the set $\mathcal{E}_{T}$ with respect to the norm \|\|$_{T}:=\|\|_{\mathcal{H}_{T}}+\|\|_{L^{2}([0, T])}$. Note that $\overline{\mathcal{E}_{T}}\|\|_{T} \subset \mathcal{H}_{T} \cap L^{2}([0, T])$.

Lemma 5.2. For any function $\psi$ in $\overline{\mathcal{E}_{T}}\|\|_{T}$ and $\eta$ in $\mathscr{S}(\mathbf{R})$, one has:

$$
<K_{+}(\eta), \psi>_{L^{2}([0, T])}=<\eta, K_{-}(\psi)>_{L^{2}([0, T])} .
$$

Proof. It is easy to check (5.4) directly in the case where $\psi$ is in $\mathcal{E}_{T}$ and $\eta$ in $\mathscr{S}(\mathbf{R})$, using (5.3). The fact that, for every $\psi \in \mathcal{E}_{T},\left\langle\psi, K_{+}()>._{L^{2}([0, T])}\right.$ belongs to $\mathscr{L}_{p}(\mathbf{R})$, for every $p$ in $\mathbb{N}^{*}$ is also clear. Thus, for every $p$ in $\mathbb{N}^{*}$, one easily sees that the map $\Psi_{p}: \psi \mapsto<\psi, K_{+}()>._{L^{2}([0, T])}$ is uniformly continuous from $\left(\mathcal{E}_{T},\|\|_{\mathcal{H}_{T}}\right)$ to $\left(\mathscr{S}_{-p}(\mathbf{R}),||_{-p}\right)$ and can then be extended uniquely to $\overline{\mathcal{E}_{T}}\|\|_{T}$ (we will denote $\Psi_{p}:=<\psi, K_{+}()>$.this extension). The same argument can be applied to the map $\Phi_{p}: \psi \mapsto<., K_{-}(\psi)>_{L^{2}([0, T])}$. The equality of $\Psi_{p}$ and $\Phi_{p}$ on $\overline{\mathcal{E}_{T}}\|\|_{T}$ from one hand, and the fact that $\left\langle\psi, K_{+}()>.=\left\langle\psi, K_{+}()>._{L^{2}([0, T])}\right.\right.$ for any $\psi$ in $\overline{\mathcal{E}_{T}}\|\|_{T}$ from the other hand allow us to conclude.

The main result of this section is the following.

Theorem 5.3. Assume that $\left.\left.H_{1}\right), H_{2}\right)$ and $\left.H_{3}\right)$ hold. Let $u$ be a process in $L^{2}\left(\Omega, \overline{\mathcal{E}}_{T}^{\|} \|_{T}\right)$, then $u$ belongs to the domain of the divergence of $G$, and $u$ is Wick-Itô integrable on $[0, T]$ wrt $G$. Moreover one has the equality

$$
\int_{0}^{T} u_{s} \delta G_{s}=\int_{0}^{T} u_{s} d^{\diamond} G_{s}
$$

Proof. The proof we give here is a generalization, to Volterra processes, of the proof provided, in the particular case of $\mathrm{fBm}$, in [Nua05, Proposition 8]. We however write it down here for reader's convenience. The fact that $\int_{0}^{T} u_{s} d^{\triangleright} G_{s}$ is well-defined has been established in Lemma 5.1 , Besides, for every fixed $\eta$ in $\mathscr{S}(\mathbf{R})$, one has: $\mathcal{L}_{1}:=S\left(\int_{0}^{T} u_{s} \delta G_{s}\right)(\eta)=S\left(\int_{0}^{T}\left(K_{-} u\right)(s) \delta W_{s}\right)(\eta)=$ $\int_{0}^{T} S\left[\left(K_{-} u\right)(s)\right](\eta) \eta(s) d s$. Note that the last equality results from the fact that the WickItô integral wrt Brownian motion generalizes the Hitsuda-Skorohod integral (see for example [HOUZ10, Theorem 2.5.9] or [Kuo96, (13.8)]). Using the previous equality, Fubini's theorem and Lemma 5.2 , one gets:

$$
\begin{aligned}
\mathcal{L}_{1} & =\int_{0}^{T} \mathbf{E}\left[\left(K_{-} u\right)(s): e^{<., \eta>}:\right] \eta(s) d s=\mathbf{E}\left[: e^{<., \eta>}:\left\langle K_{-} u, \eta>_{L^{2}([0, T])}\right]\right. \\
& =\mathbf{E}\left[: e^{<., \eta>}:\left\langle u, K_{+}(\eta)>_{L^{2}([0, T])}\right]=\int_{0}^{T} \mathbf{E}\left[u_{s}: e^{<., \eta>}:\right] K_{+}(\eta)(s) d s .\right.
\end{aligned}
$$


It then remains to use (ii) of Theorem [3.6 as well as (5.3) to obtain:

$$
\mathcal{L}_{1}=\int_{0}^{T} S\left(u_{s}\right)(\eta) S\left(W_{s}^{(G)}\right)(\eta) d s=S\left(\int_{0}^{T} u_{s} \diamond W_{s}^{(G)} d s\right)(\eta) .
$$

We hence have shown, for every $\eta$ in $\mathscr{S}(\mathbf{R})$, the equality $S\left(\int_{0}^{T} u_{s} \delta G_{s}\right)(\eta)=S\left(\int_{0}^{T} u_{s} d^{\diamond} G_{s}\right)(\eta)$. The injectivity of $S$-transform (see $(i)$ of Lemma 2.5) allows us to conclude.

Example 5.4 (The case of $\mathrm{fBm})$. Let $T>0$. For any $H$ in $(0,1)$, define $B_{t}^{H}:=\int_{0}^{t} K_{H}(t, s) d W_{s}$, where the kernel $K_{H}$ is defined in [Nua05, (13)]. $B^{H}$ is an fBm of Hurst index H. Moreover the process $B^{H}$ fulfills H1), H2) and H3). This implies in particular that the Wick-Ito integral $\int_{0}^{T} B_{s}^{H} d^{\diamond} B_{s}^{H}$ exists, for any $H$ in $(0,1)$. We know moreover, thanks to Example 3.14, that the equality $\int_{0}^{T} B_{s}^{H} d^{\diamond} B_{s}^{H} \stackrel{\text { a.s. }}{=} \frac{1}{2}\left(\left(B_{T}^{H}\right)^{2}-T^{2 H}\right)$ is true for any $H$ in $(0,1)$. On the other hand, the divergence integral wrt $B^{H}$ is only defined and developed in [AMN01, Section 8] or in [Nua06, Section 6] for $H>1 / 4$, as we mentioned in the introduction. One moreover knows that $B^{H}$ does not belong to $\mathbb{D}$ om $\left(\delta_{B^{H}}\right)$ when $H<1 / 4$ and one has to use the extended divergence integral wrt $f B m$ developed in [CN05].

Corollary 5.5. The set $\left\{\int_{0}^{T} f(s) \delta G_{s}, f \in \mathcal{H}_{T}\right\}$ of Wiener divergence integral wrt $G$, coincide with the set $\Theta_{G}:={\overline{\left\{\int_{0}^{T} f(s) d^{\diamond} G_{s}, f \in \mathcal{E}_{T}\right\}}}^{\left(L^{2}\right)}$ of Wick-Itô Wiener integrals wrt $G$.

Proof. The equality $\left\{\int_{0}^{T} f(s) d^{\diamond} G_{s}, f \in \mathcal{E}_{T}\right\}=\left\{\int_{0}^{T} f(s) \delta G_{s}, f \in \mathcal{E}_{T}\right\}$ is obvious, in view of (5.5). Besides, the equality $\left\{\int_{0}^{T} f(s) \delta G_{s}, f \in \mathcal{H}_{T}\right\}=\overline{\left\{\int_{0}^{T} f(s) \delta G_{s}, f \in \mathcal{E}_{T}\right\}}{ }^{\left(L^{2}\right)}$ results from Meyer inequalities (see [AMN01, (5)] for example).

Remark 10. 1. In many cases (such as for fBm) the equality $\overline{\mathcal{E}}_{T}^{\|} \|_{T}=\mathcal{H}_{T} \cap L^{2}([0, T])$ is clear. In these situations Theorem 5.3 in a way that makes clear how the wick Itô integral wrt $G$ generalizes the divergence one. Indeed, when equality $\overline{\mathcal{E}}_{T}^{\|} \|_{T}=\mathcal{H}_{T} \cap L^{2}([0, T])$ holds one just has to assume that $\left.\left.H_{1}\right), H_{2}\right)$ and $\left.H_{3}\right)$ hold. Hence, for any process $u$ in $L^{2}\left(\Omega, L^{2}([0, T])\right)$, if $u$ belongs to the domain of the divergence of $G$, then $u$ is Wick-Itô integrable on $[0, T]$ wrt $G$. Moreover Equality (5.5) holds.

2. In view of the previous corollary, we see that one just has to extend the notion of Wiener integral given in Definition 1, and call Wiener integral wrt $G$ in $\mathscr{G}$, any element of $\Theta_{G}$, if one wants that our set of Wiener integrals is the same that the one of [AMN01].

3. If Theorem 5.3 clearly states that the Wick-Itô integral has a bigger set of integrands than the divergence type integral developed in [AMN01], assuming they both belong to $L^{2}\left(\Omega, \overline{\mathcal{E}}_{T}\|\|_{T}\right)$, one may wonder if this fact remains true outside $L^{2}\left(\Omega, \overline{\mathcal{E}}_{T}\|\|_{T}\right)$. While this remains an open problem, here is what we can still say about it. The set $\mathcal{H}_{T}$ may contains generalized functions (for example, one can see [Nua06, p.280] or [LLV14, Proposition 2.11] in the case where $G$ is a fBm). When this happens (i.e. when, for almost every $\omega$ in $\Omega, u(\omega)$ is a generalized function which belongs to $\mathcal{H}_{T}$ and which is not a function), $\int_{0}^{T} u_{s} \delta G_{s}$ has still a meaning and belongs to $L^{2}(\Omega)$. On the contrary, $\int_{0}^{T} u_{s} d^{\diamond} G_{s}$ can only be defined if $s \mapsto u_{s}$ is a function (an $\left(\mathcal{S}^{*}\right)$-valued function but still a function). Define the space

$$
\Lambda:=\left\{u \in L^{2}\left(\Omega ; \mathcal{H}_{T}\right) ; u \text { is Wick-Itô integrable wrt } G \text { and such that } \int_{0}^{T} u_{s} d^{\diamond} G_{s} \in L^{2}(\Omega)\right\} .
$$

A consequence of what we stated above is that the inclusion $\mathbb{D}$ om $\left(\delta_{G}\right) \subset \Lambda$ is not true. Note that the inclusion $\Lambda \subset \mathbb{D o m}\left(\delta_{G}\right)$ does not hold either. Indeed, if one considers again, as process $G$, the $f B m B^{H}$, as we did in Example 5.4, we know that $B^{H}$ belongs to $\Lambda$ for every $H$ in $(0,1)$, while $B^{H}$ does not belong to $\mathbb{D} o m\left(B^{H}\right)$ if $H$ is in $(0,1 / 4)$. Finally, the only thing one can say in general is that we have the dense inclusion $L^{2}\left(\Omega, L^{2}([0, T])\right) \cap \mathbb{D}$ om $\left(\delta_{G}\right) \subset \Lambda$. 


\subsubsection{Comparison with the divergence type integral of [MV05]}

The comparison between Wick-Itô stochastic integral and the one defined in [MV05] is easier, in view of Theorems 3.4 and 5.3 . Indeed, one has the following result.

Theorem 5.6. For any process $u$ such that the (extended) Skorohod integral wrt $G$, on $[0, T]$, defined in [MV05], exists, then u is also Wick-Itô integrable wrt $G$, on $[0, T]$. Moreover one has the equality

$$
\text { MV05- } \int_{0}^{T} u_{s} \delta G_{s}=\int_{0}^{T} u_{s} d^{\diamond} G_{s}
$$

where MV05] - $\int_{0}^{T} u_{s} \delta G_{s}$ denotes the (extended) Skorohod integral of u wrt G, defined in MV05.

Remark 11. Note that, in this case, one does not have to make any additional assumptions (such as H1, H2 or H3) nor that the equality $\overline{\mathcal{E}_{T}} \|_{T}=\mathcal{H}_{T} \cap L^{2}([0, T])$ holds. Moreover, and as we stated in the introduction, this theorem as well as Theorem 3.4 show that the stochastic integral wrt to $\mathscr{V}_{\gamma}$ - processes, built in [MV05], is a particular case of the Wick-Itô stochastic integral we provide here. This means that for every $\mathscr{V}_{\gamma}$ - process $\widetilde{B}^{\gamma}$, and every stochastic process $X$, such that the integral of $X$ wrt $\widetilde{B}^{\gamma}$ exists in the sense defined in [MV05], the Wick-Ito stochastic integral of $X$ wrt $\widetilde{B}^{\gamma}$ exists. Moreover they are equal. Finally, this also allows us to deal with non continuous Gaussian processes, as it is the case in [MV05].

Proof. Using notations of Theorem 3.4, Lemma 2.3 and Cauchy-Schwarz inequality one gets, for every $\eta$ in $\mathscr{S}(\mathbf{R})$ and every integer $q \geq 3$ :

$$
\int_{0}^{T}\left|S\left(u_{s}\right)(\eta) S\left(W_{s}^{(G)}\right)(\eta)\right| d s \leq\left(\int_{0}^{T}\left\|u_{s}\right\|_{0}^{2} d s\right)^{1 / 2}\left(\int_{0}^{T}\left|\Phi^{\prime}(s)\right|_{-q}^{2} d s\right)^{1 / 2} e^{|\eta|_{q}^{2}}
$$

Since both quantities $\|u\|_{L^{2}\left(\Omega, L^{2}([0, T])\right)}^{2}$ and $\int_{0}^{T}\left|\Phi^{\prime}(s)\right|_{-q}^{2} d s$ are finite (by assumption for the first one and as a consequence of Theorem 3.4 ofr the second one), Theorem 2.6 applies and establishes the existence of $\int_{0}^{T} u_{s} d^{\diamond} G_{s}$. Besides, since in this case the extended domain of the [MV05] - Skorohod integral is, by its very definition (see [MV05, Def. 27]) a subset of $L^{2}\left(\Omega, L^{2}([0, T])\right.$, one can use the exact same proof as the one of Theorem 5.3, one just has to change therein $K_{-}$ (resp. $\left.K_{+}\right)$by $K_{\gamma}^{*}\left(\right.$ resp. $\left.K_{\gamma}^{*, a}\right)$ and note that the equality given in [MV05, Remark 12] has now the role played by Equality (5.4), in the proof of Theorem 5.3. The only thing which remains to be shown is that $\mathscr{S}(\mathbf{R}) \subset \mathcal{H}^{\prime}$, where $\mathcal{H}^{\prime}:=\left\{f \in L^{2}([0, T]), K_{\gamma}^{*, a} f \in L^{2}([0, T])\right\}$. This latter inclusion results from [MV05, Proposition 15] (one just has to take therein $\eta(s):=s^{\alpha}$ on $\mathbf{R}_{+}^{*}$, with $\alpha \in(1 / 2,1)$ and $\eta(0):=0$ and then show that $\mathscr{S}(\mathbf{R}) \subset C^{\eta}$, where $C^{\eta}$ has been defined in Example 3.9 .

Note that the results provided in both Thm. 5.6 and Thm. 3.16 allow us to think that one could solve some linear stochastic evolution equations driven by infinite dimensional Gaussian processes.

In MV05 the set of Gaussian processes is smaller than $\mathscr{G}$. In NT06 the class of Gaussian processes considered is a little bit restrictive6, (see [NT06, (2), (3) \& (4)]). Moreover, since our stochastic calculus is carried out within the framework of the White Noise Theory, our stochastic integral does not have to be extended, once it has been built, in order that the set of

\footnotetext{
${ }^{6}$ Moreover, while the Wick product is used to define a stochastic integral in NT06, the space of stochastic distributions (which is the natural set on which one can use Wick product) is not used at all. This latter is crucial to derive occupation time formulas for local times, as we will show in Leb17.

${ }^{7}$ Besides, if CN05 provides a method (that has been used in MV05) to extend the divergence type integral wrt $\mathrm{fBm}$, this leads to require much more regularity on the function $f$, to provide an Itô formula 8 .
} 
integrands is not empty or not too small, as it is the case for divergence type integral (see MV05, Remark 25 \& p.407] and [CN05]). Indeed, it happens that the Gaussian process is not even itself integrable i.e. that $\int G_{s} \delta G_{s}$ does not exist, (e.g. in [AMN01] when $G$ is a fBm with $H \leq 1 / 4$ or when $G$ is the process considered in [MV05]). Note that the same phenomenon happens alsd 9 in [LN12. A general way to extend the divergence integral for Volterra processes, assuming it exists, has been provided in [LN05]. However 10 , an Itô formula for extended divergence integral has not been provided in the same time for general Volterra processes. Finally, our stochastic calculus is an extension to general Gaussian processes of the stochastic calculus built, wrt $\mathrm{fBm}$ in [EVdH03, BSØW04, Ben03a] and wrt mBm in [LLV14, Leb13, LLVH14].

\subsection{Comparison with Itô Integral}

The goal of this section is to compare the Wick-Itô integral wrt $G$ to the Itô integral wrt $G$, when $G$ is a (Gaussian) semimartingale. In this subsection we still assume that $\mathscr{R}=[0, T]$. Since the line of reasoning we are following would be similar if $t \mapsto G_{t}$ would not be continuous, we will assume, in this subsection, that $G$ is continuous. Denote, for every $t$ in $[0, T], \mathcal{U}_{t}$ the complet 110 -field defined by $\mathcal{U}_{t}:=\sigma\left(\left\{G_{s} ; 0 \leq s \leq t\right\}\right)$ and denote $\mathcal{U}$ the filtration $\left(\mathcal{U}_{t}\right)_{t \in[0, T]}$. In this subsection one then assumes that $G=\left(G_{t}\right)_{t \in[0, T]}$ is a continuous centered Gaussian $\mathcal{U}$ semimartingale of the form (1.1), which fulfills Assumption $(\mathscr{A})$. Let us recall first the following result, that describes the structure of Gaussian semimartingales.

Proposition 5.7. [Str83, Prop. 2 \& Thm 1 ] The Gaussian $\mathcal{U}$-semimartingale $G$ is a special $\mathcal{U}$-semimartingale: i.e. for almost every $(t, \omega)$ in $[0, T] \times \Omega$, one can write:

$$
G_{t}=M_{t}+A_{t}
$$

where $M:=\left(M_{t}\right)_{t \in[0, T]}$ is a centered $\mathcal{U}$-martingale and $A:=\left(A_{t}\right)_{t \in[0, T]}$ is a centered $\mathcal{U}$ predictable process of bounded variations. Moreover, $M$ and $A$ both belong to the same Gaussian Hilbert space as $G$. In addition, the function of quadratic variation of $G$, denoted $t \mapsto\langle G\rangle_{t}$, is deterministic and $M$ is bounded in $L^{p}$, for every positive real $p$.

Denote $\mathcal{T}:=\left(\mathcal{T}_{t}\right)_{t \in[0, T]}$ the filtration, defined by $\mathcal{T}_{t}:=\sigma\left(\left\{B_{s} ; 0 \leq s \leq t\right\}\right)$, which we suppose complete (if it is not the case we complete it and still denote it $\mathcal{T}_{t}$ ). Through this subsection, we will denote $\mathscr{I}_{G}(X):=\int_{0}^{T} X_{s} d G_{s}$ the Itô (resp. $\mathscr{J}_{G}(X):=\int_{0}^{T} X_{s} d^{\diamond} G_{s}$ the Wick-Itô) integral of $X$ wrt $G$ on $[0, T]$, when it exists. For any continuous martingale $M:=\left(M_{t}\right)_{t \in[0, T]}$, bounded in $\left(L^{2}\right)$ and such that $M_{0}=0$, denote $L^{2}(M):=L^{2}\left([0, T] \times \Omega, \mathcal{P}, d \mu d<M>_{s}\right)$ the space of progressively measurable processes $K$ such that:

$$
\|K\|_{L^{2}(M)}^{2}:=\mathbf{E}\left[\int_{0}^{T} K_{s}^{2} d<M>_{s}\right],
$$

where $\mathcal{P}$ denote the progressive $\sigma$-field with respect to $\mathcal{U}$. The following result will be used in order to establish Point $(i)$ of Proposition 5.9 below.

Lemma 5.8. Let $G$ be a Gaussian martingale that fulfills Assumption $(\mathscr{A})$ and let $f$ be a $C^{1,2}([0, T] \times \mathbf{R}, \mathbf{R})$ function. Denote $F(t, x):=\frac{\partial f}{\partial x}(t, x)$ and define $X_{t}:=\frac{\partial f}{\partial x}\left(t, G_{t}\right)$. If $R$ and $f$ both fulfill conditions of Theorem 4.4, then one has the following equality:

$$
\text { a.s. } \int_{0}^{T} X_{s} d G_{s}=\int_{0}^{T} X_{s} d^{\diamond} G_{s} .
$$

\footnotetext{
${ }^{9}$ In this latter case, the extended domain and the initial one are not comparable (see [LN12, p. 383]).

${ }^{10}$ and if one excepts MV05, the results of which we fully generalize in this paper.

${ }^{11}$ If $\mathcal{U}_{t}$ is not complete, we complete it and still denote it $\mathcal{U}_{t}$.
} 
In particular one has the equality:

$$
\text { a.s. } \int_{0}^{T} F\left(s, G_{s}\right) d G_{s}=\int_{0}^{T} F\left(s, G_{s}\right) d^{\diamond} G_{s} .
$$

Proof of Lemma 5.8. $G$ being a Gaussian martingale, one gets $\langle G\rangle_{t}=R_{t}$ almost surely, for every $t$ in $[0, T]$. Since $R$ and $f$ both fulfill conditions of Theorem 4.4, one gets, using both Itô formulas (4.5) and [RY99, Theorem 3.3]): $\Delta_{G}(X):=\mathscr{I}_{G}(X)-\mathscr{J}_{G}(X)=0$, which in particular implies that: $\int_{0}^{T} F\left(s, G_{s}\right) d G_{s}=\int_{0}^{T} F\left(s, G_{s}\right) d^{\diamond} G_{s}$.

The main result of this subsection is the following.

Proposition 5.9. 1. Assume that $G$ is Gaussian martingale, adapted to the filtration $\mathcal{T}$. Let $X$ in $L^{2}(G)$ such that $X_{t} \in\left(L^{2}\right)$, for every $t$ in $[0, T]$, and such that $(X, G)$ satisfies condition $(\mathcal{I})$ (given in Section [3.5). Define, for every $s$ in $[0, T], U_{s}:=\inf \left\{t ; R_{t}>s\right\}$. Let us write the following conditions:

a) the map $t \mapsto R_{t}$ is strictly increasing and continuous on $[0, T]$ and such that $U$ is absolutely continuous on $[0, T]$.

b) $G_{t_{2}}-G_{t_{1}}$ is independent of $\mathcal{T}_{t_{1}}$, for every $0 \leq t_{1}<t_{2} \leq T$.

If one the two conditions a) or b) is fulfilled then the map $t \mapsto X_{t}$ is both $d G$-integrable and Itô-integrable, on $[0, T]$. Moreover we have the equality:

$$
\int_{0}^{T} X_{s} d G_{s}=\int_{0}^{T} X_{s} d^{\diamond} G_{s}
$$

2. If the semimartingale $G$ is not a Gaussian martingale, then Equality (5.9) does not hold in general, assuming both integral $\int_{0}^{T} X_{s} d G_{s}$ and $\int_{0}^{T} X_{s} d^{\diamond} G_{s}$ do exist.

Note that the condition $X_{t} \in\left(L^{2}\right)$, for every $t$ in $[0, T]$ is only a slight reinforcement of the assumption $X \in L^{2}(G)$.

Proof. 1. Let $G$ and $X$ be of the form described in point 1. above. We still denote $\mathscr{I}_{G}(X):=$ $\int_{0}^{T} X_{s} d G_{s}$ the Itô (resp. $\mathscr{J}_{G}(X):=\int_{0}^{T} X_{s} d^{\diamond} G_{s}$ the Wick-Itô) integral of $X$ wrt $G$ on $[0, T]$, when it exists. The existence of $\mathscr{I}_{G}(X)$ is clear and the existence of $\mathscr{J}_{G}(X)$ is obvious, in view of Theorem 3.10 ,

1.a) $G$ being a martingale, it can be written, according to Dubins-Schwarz theorem as $G_{u}=$ $B_{R_{u}}$, for some Brownian motion $B$ and for every $u$ in $[0, T]$. Of course we also have the equalities $B_{t}=G_{U_{t}}$ and $U_{R_{t}}=t$, for every $t$ in $[0, T]$ since $R$ is strictly increasing. It is then clear that:

$$
\mathscr{I}_{G}(X)=\int_{0}^{T} X_{s} d G_{s}=\int_{0}^{T} X_{s} d B_{R_{s}}=\int_{0}^{T} X_{U_{R_{s}}} d B_{R_{s}}=\int_{0}^{R_{T}} X_{U_{t}} d B_{t}=\int_{0}^{R_{T}} X_{U_{t}} d^{\diamond} B_{t} .
$$

For every $\eta$ in $\mathscr{S}(\mathbf{R})$, we can then write $S\left(\mathscr{I}_{G}(X)\right)(\eta)=\int_{0}^{R_{T}} S\left(X_{U_{t}}\right)(\eta)<\delta_{t}, \eta>d t$. Besides, we have the equality $<\mathbf{1}_{[0, t]}, \eta>_{L^{2}(\mathbf{R})}=\mathbf{E}\left[B_{t}<., \eta>\right]=\mathbf{E}\left[G_{U_{t}}<., \eta>\right]=<g_{U_{t}}, \eta>_{L^{2}(\mathbf{R})}$. Since $U$ is absolutely continuous on $[0, T]$ so is $\left.s \mapsto<g_{U_{s}}, \eta\right\rangle$. Thus this yields to:

$$
S\left(\mathscr{I}_{G}(X)\right)(\eta)=\int_{0}^{R_{T}} S\left(X_{U_{t}}\right)(\eta) d<g_{U_{t}}, \eta>.
$$

On the other hand, we have: $S\left(\mathscr{J}_{G}(X)\right)(\eta)=\int_{0}^{T} S\left(X_{s}\right)(\eta) d<g_{s}, \eta>=: \int_{0}^{T} f_{\eta}(s) d A_{s}^{(\eta)}$, where we have set $A_{s}^{(\eta)}:=<g_{s}, \eta>$ and $f_{\eta}=S(X$.)( $\eta)$. Applying [RY99, Proposition 4.10] to 
the positive and then the negative part of $f_{\eta}$ we get $\int_{0}^{T} f_{\eta}(s) d A_{s}^{(\eta)}=\int_{0}^{R_{T}} f_{\eta}\left(U_{s}\right) d A_{U_{s}}^{(\eta)}$ which entails that:

$$
S\left(\mathscr{J}_{G}(X)\right)(\eta)=\int_{0}^{R_{T}} f_{\eta}\left(U_{s}\right) d A_{U_{s}}^{(\eta)}=\int_{0}^{R_{T}} S\left(X_{U_{t}}\right)(\eta) d<g_{U_{t}}, \eta>
$$

and ends the proof in this case.

1.b) The proof of the equality $\mathscr{I}_{G}(X)=\mathscr{J}_{G}(X)$ is obtained by following exactly the same three steps as in the proof of [Kuo96, Theorem 13.12], in which the equality $\mathscr{I}_{B}(X)=\mathscr{J}_{B}(X)$ is established ( $B$ being a Brownian motion). One then just has to substitute in there the process $\varphi$ by $X$, to replace $\mathbf{1}_{\left[t_{1}, t_{2}\right)}$ by $g_{t_{2}}-g_{t_{1}}$ in the first step, and noticing that one can find, for any process $X$ in $L^{2}(G)$, a sequence $\left(X_{n}\right)_{n \in \mathbf{N}}$ of simple processes such that $\lim _{n \rightarrow+\infty} \mathscr{I}_{G}\left(X_{n}\right)=\mathscr{I}_{G}(X)$, where the convergence holds in $\left(L^{2}\right)$. We will only write down here the first of these three steps; in order, first to make clear the differences with the case where $G=B$, and second, to translate the proof of [Kuo96, Theorem 13.12] in the notations we use in this paper. Let $\left(t_{1}, t_{2}\right)$ be in $[0, T]^{2}$ such that $0 \leq t_{1}<t_{2} \leq T$ and assume that $X_{t}:=X_{t_{1}} \mathbf{1}_{\left(t_{1}, t_{2}\right]}$, where $X_{t_{1}}$ is $\mathcal{U}_{1}$-mesurable. Let $X_{t_{1}}=\sum_{n=0}^{+\infty} I_{n}\left(f_{t_{1}}^{(n)}\right)$ be the chaos decomposition of $X_{t_{1}}$. By definition of Itô integral, and using the identity: $\left.I_{n}(k) I_{1}(l)=I_{n+1}(k \otimes l)+n I_{n-1}(<k, l\rangle_{L^{2}(\mathbf{R})}\right)$, where $\widehat{\otimes}$ denotes the symmetric tensor product and which is valid for every: $n$ in $\mathbf{N}^{*}$, symmetric function $k$ in $L^{2}\left(\mathbf{R}^{n}\right)$ and $l$ in $L^{2}(\mathbf{R})$, onøogets:

$$
\begin{aligned}
\mathscr{I}_{G}(X)=X_{t_{1}}\left(G_{t_{2}}-G_{t_{1}}\right) & =\sum_{n=0} I_{n}\left(f_{t_{1}}^{(n)}\right) I_{1}\left(g_{t_{2}}-g_{t_{1}}\right) \\
& =\sum_{n=0}^{+\infty}\left(I_{n+1}\left(f_{t_{1}}^{(n)} \widehat{\otimes}\left(g_{t_{2}}-g_{t_{1}}\right)\right)+n I_{n-1}\left(<f_{t_{1}}^{(n)}, g_{t_{2}}-g_{t_{1}}>_{L^{2}(\mathbf{R})}\right)\right) .
\end{aligned}
$$

Lemma 3.11 of [Kuo96] applies here since $\mathcal{U}$ is included in $\mathcal{T}$. One then knows that, for every $n$ in $\mathbf{N}^{*}, f_{t_{1}}^{(n)}$ is equal to 0 almost everywhere on $[0, T]^{n} \backslash\left[0, t_{1}\right]^{n}$. Moreover, since $G_{t_{2}}-G_{t_{1}}$ is independent of $\mathcal{T}_{t_{1}}$, it is clear that $\left\langle f_{t_{1}}^{(n)}, g_{t_{2}}-g_{t_{1}}\right\rangle_{L^{2}(\mathbf{R})}=0$, for every $n$ in $\mathbf{N}^{*}$. Using Proposition 2.4, we get, for any $\eta$ in $\mathscr{S}(\mathbf{R})$,

$$
\begin{aligned}
S\left(\mathscr{I}_{G}(X)\right)(\eta) & =S\left(\sum_{n=0}^{+\infty}\left(I_{n+1}\left(f_{t_{1}}^{(n)} \widehat{\otimes}\left(g_{t_{2}}-g_{t_{1}}\right)\right)\right)(\eta)=\sum_{n=0}^{+\infty}<f_{t_{1}}^{(n)} \widehat{\otimes}\left(g_{t_{2}}-g_{t_{1}}\right), \eta^{\otimes(n+1)}>\right. \\
& =\sum_{n=0}^{+\infty}<f_{t_{1}}^{(n)}, \eta^{\otimes n}><g_{t_{2}}-g_{t_{1}}, \eta>=S\left(X_{t_{1}}\right)(\eta) S\left(G_{t_{2}}-G_{t_{1}}\right)(\eta)=S\left(\mathscr{J}_{G}(X)\right)(\eta) .
\end{aligned}
$$

The injectivity of S-transform then allows us to write $\mathscr{I}_{G}(X)=\mathscr{J}_{G}(X)$.

2. In view of Proposition 5.7, three cases are possible for the structure of the semimartingale $G$. The case where $G$ is a martingale has been treated in Point $\mathbf{1}$ below. Our goal here is to exhibit, when $G$ is not a Gaussian martingale, some general and simple examples for which $\mathscr{I}_{G}(X)$ and $\mathscr{J}_{G}(X)$ both exist and are different. Let $f$ be a $C^{1,2}([0, T] \times \mathbf{R}, \mathbf{R})$ function. Assume that both $R$ and $f$ fulfill conditions of Theorem 4.4. Denote $Y \equiv 0$ when the process $Y:=\left(Y_{t}\right)_{t \in[0, T]}$ is such that

$$
Y_{t}(\omega)=0, \quad \forall(\omega, t) \in \Omega^{\prime} \times[0, T],
$$

where $\Omega^{\prime}$ is measurable subset such that $\mu\left(\Omega^{\prime}\right)=1$. We will denote $Y \not \equiv 0$ when (5.10) is not satisfied.

The case where $M \equiv 0$ being easier we will assume that $A \not \equiv 0$ and $M \not \equiv 0$ in (5.7). Assume that both $M$ and $A$ are continuous and that there exists a map $t \mapsto g_{t}^{(1)}$ from $[0, T]$ into $L^{2}(\mathbf{R})$ such that $g^{(1)}$ fulfills Assumption $(\mathscr{A})$ and such that $M_{t}=<., g_{t}^{(1)}>$ almost surely, for every $t$ 
in $[0, T]$. Let us compare $\mathscr{I}_{G}(G)$ and $\mathscr{J}_{G}(G)$. The existence of $\mathscr{I}_{G}(G)$ is clear. Moreover, using classical Itô formula, one gets:

$$
\mathscr{I}_{G}(G)=\int_{0}^{T} M_{s} d M_{s}+\int_{0}^{T} A_{s} d A_{s}+A_{T} M_{T}
$$

The existence of $\mathscr{J}_{G}(G)$ is clear in view of Example 3.14, Moreover, using again Example 3.14, the fact that $M$ is bounded in $\left(L^{2}\right)$ as well as an integration by parts, one gets:

$$
\mathscr{J}_{G}(G)=\int_{0}^{T} M_{s} d^{\diamond} M_{s}+\int_{0}^{T} A_{s} d^{\diamond} A_{s}+A_{T} \diamond M_{T} .
$$

Classical Itô formula, Example 3.14, (5.8) and, finally, Propsotion 5.7 and Proposition 2.4 yields:

$$
\begin{aligned}
\Theta_{G}(G) & :=\mathscr{I}_{G}(G)-\mathscr{J}_{G}(G)=\int_{0}^{T} A_{s} d A_{s}-\int_{0}^{T} A_{s} d^{\diamond} A_{s}+A_{T} M_{T}-A_{T} \diamond M_{T} \\
& =2^{-1} \mathbf{E}\left[A_{T}^{2}\right]+A_{T} M_{T}-A_{T} \diamond M_{T}=2^{-1} \mathbf{E}\left[A_{T}^{2}\right]-\mathbf{E}\left[A_{T} M_{T}\right] .
\end{aligned}
$$

It is then easy to find a finite variation processes $A$, as well as a positive real $T$, and choose the map $g^{(1)}$, that defines the Gaussian martingale $M$, such that: $2^{-1} \mathbf{E}\left[A_{T}^{2}\right]-\mathbf{E}\left[A_{T} M_{T}\right] \neq 0$.

Remark 12. 1. In the particular case where there exists a function $f: \mathbf{R} \rightarrow \mathbf{R}$, which belongs to $L^{2}(\mathbf{R})$, such that $G_{t}:=\int_{0}^{t} f(u) d B_{u}$, for every $t$ in $[0, T]$, a.s., then all the assumptions of Propoistion 5.9 are reduced to $X$ belongs to $L^{2}(G)$. Note also that one recovers in particular, the result of [Kuo96, Theorem 13.12], that is $\int_{0}^{1} X_{s} d^{\diamond} B_{s}=\int_{0}^{1} X_{s} d B_{s}$, for every $X$ in $L^{2}(B)$. 2. One may also remark, from what we stated in the previous sections, that the existence of $\int_{0}^{1} X_{s} d^{\diamond} G_{s}$ does not imply the existence $\int_{0}^{1} X_{s} d G_{s}$. Conversely the existence of $\int_{0}^{1} X_{s} d G_{s}$ does not imply the existence $\int_{0}^{1} X_{s} d^{\diamond} G_{s}$. Three natural questions then arise in this framework;

(i) If $\int_{0}^{T} X_{s} d G_{s}$ exist, on which conditions on $X$ the integral $\int_{0}^{T} X_{s} d^{\triangleright} G_{s}$ will exist?

(ii) If $\int_{0}^{T} X_{s} d^{\triangleright} G_{s}$ exist, on which conditions on $X$ the integral $\int_{0}^{T} X_{s} d G_{s}$ will exist?

(iii) When both the integrals $\int_{0}^{T} X_{s} d^{\diamond} G_{s}$ and $\int_{0}^{T} X_{s} d G_{s}$ do exist, what is the exact link bet ween them?

In order to answer properly to these three questions one needs to use the operators $D_{g_{t}}$ and $D_{g_{t}}^{*}$, defined in [Kuo96, Chap 9], and express both our Wick-Itô integral and the Itô integral using these operators. Since this would lead us too far from the goal of this present work, we will therefore give the answer to these questions in a future work.

3. In view of Lemma 5.8, it seems that Equality (5.9) remains true under weaker assumptions than the one proposed in Proposition 5.9. However, extend (5.9) under weaker assumptions is an open problem.

4. Of course one can limit our definition of Wick-Itô integral to Gaussian martingales only. Then, and as it is the case for Itô integral, one can extend the definition of Wick-Itô integral wrt $G$ to the case where $G$ is a Gaussian semimartingale, by simply setting:

$$
\int_{0}^{T} X_{s} d^{*} G_{s}:=\int_{0}^{T} X_{s} d^{\diamond} M_{s}+\int_{0}^{T} X_{s} d A_{s}
$$

where $M$ (resp. A) denotes the martingale (resp. the bounded variation process) given by (5.7), and where $M$ is assumed to be of the form (1.1) and fulfills Assumption $(\mathscr{A})$. $\int_{0}^{T} X_{s} d^{*} G_{s}$ will then be defined as soon as each member of the right hand side of (5.12) will exist. The Itô integral $\int_{0}^{T} X_{s} d A_{s}$, in the right hand side of (15.12), offers also the advantage, on $\int_{0}^{T} X_{s} d^{\diamond} A_{s}$ of being defined $\omega$ by $\omega$ since it is Stieljes integral. 


\section{Acknowledgments}

I want to express my deep gratitude to Jacques Lévy Véhel for his advices and for the very stimulating discussions we had about this work. I also want to thank Professor T. Hida for his warm welcome at the University of Nagoya, where a part of this paper was written, as well as Professor L. Chen and the Institute for Mathematical Sciences of Singapore (NUS), where another part of this paper was written. I also thanks the Associate Editor as well as the anonymous referee for his remarks that greatly improve the quality of this paper and especially Section 4.2

This work is dedicated to the memory of Professor Marc Yor.

\section{Appendix}

\section{A. Bochner Integral}

The following notions about Bochner integral come from [HP57, p.72, 80 and 82] and [Kuo96, p.247].

Definition 3. Bochner integral [Kuo96, p.247] Let I be a Borelian subset of $\mathbf{R}$ endowed with the Lebesgue measure. One says that $\Phi: I \rightarrow(\mathcal{S})^{*}$ is Bochner integrable on I if it satisfies the two following conditions: $1 \Phi$ is weakly measurable on $I$ i.e $u \mapsto \ll \Phi_{u}, \varphi \gg$ is measurable on I for every $\varphi$ in $(\mathcal{S})$.

$2 \exists p \in \mathbb{N}$ such that $\Phi_{u} \in\left(\mathcal{S}_{-p}\right)$ for almost every $u \in I$ and $u \mapsto\left\|\Phi_{u}\right\|_{-p}$ belongs to $L^{1}(I)$.

The Bochner-integral of $\Phi$ on $I$ is denoted $\int_{I} \Phi_{s} d s$.

Proposition A.1. If $\Phi: I \rightarrow(\mathcal{S})^{*}$ is Bochner-integrable on $I$ then there exists an integer $p$ such that $\left\|\int_{I} \Phi_{s} d s\right\|_{-p} \leq \int_{I}\left\|\Phi_{s}\right\|_{-p} d s$. Moreover $\Phi$ is also Pettis-integrable on I and both integrals coincide on I.

Remark 13. The previous proposition shows that there is no risk of confusion by using the same notation for both Bochner and Pettis integrals.

Theorem A.2. Let $p \in \mathbb{N}$ and $\left(\Phi^{(n)}\right)_{n \in \mathbb{N}}$ be a sequence of processes from $I$ to $(\mathcal{S})^{*}$ such that $\Phi_{u}^{(n)} \in\left(\mathcal{S}_{-p}\right)$ for almost every $u \in I$ and for every $n$. Assume moreover that $\Phi^{(n)}$ is Bochner-integrable on $I$, for every $n$, and that $\lim _{(n, m) \rightarrow(+\infty,+\infty)} \int_{I}\left\|\Phi_{s}^{(m)}-\Phi_{s}^{(n)}\right\|_{-p} d s=0$. Then there exists an $(\mathcal{S})^{*}$-process (almost surely $\left(\mathcal{S}_{-p}\right)$-valued), denoted $\Phi$, defined and Bochner-integrable on $I$, such that

$$
\lim _{n \rightarrow+\infty} \int_{I}\left\|\Phi_{s}-\Phi_{s}^{(n)}\right\|_{-p} d s=0
$$

Furthermore, if there exists an $(\mathcal{S})^{*}$-process, denoted $\Psi$, which verifies (A.1), then $\Psi_{s}=\Phi_{s}$ for a.e. $s$ in I. Finally one has $\lim _{n \rightarrow+\infty} \int_{I} \Phi_{s}^{(n)} d s=\int_{I} \Phi_{s} d s$, where the equality and the limit both hold in $(\mathcal{S})^{*}$.

\section{B. Proof of Theorem 3.4}

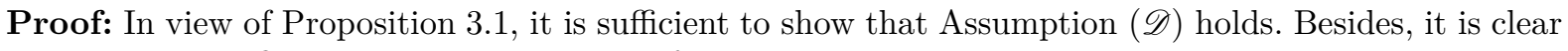
that $\Phi$ is well defined on $\mathbf{R}_{+}$since one has, for every $t$ in $\mathbf{R}_{+}$, the equality:

$$
\Phi_{t}=E(t) \cdot \delta_{0}-\left(\mathbf{1}_{[0, t)} \cdot E(t-\cdot)\right)^{\prime} .
$$

It is clear that function $E$ (resp. $\mathscr{E}$ ) is increasing, differentiable on $\mathbf{R}_{+}^{*}$ and continuous on $\mathbf{R}_{+}$(resp. increasing and of class $C^{1}$ on $\mathbf{R}_{+}$). Equality (B.1) together with the properties of $E$ entail that $\Phi$ is continuous at $t=0$. Let us now establish Equality (3.5). For every $t$ in $\mathbf{R}_{+}^{*}, \varphi$ in $\mathscr{S}(\mathbf{R})$, and $r>0$, denote $I_{r}:=<\frac{\Phi_{t+r}-\Phi_{t}}{r}, \varphi>$. Using the change of variable formula, an easy computation gives us:

$$
\begin{aligned}
& I_{r}=\frac{1}{r}\left(\int_{0}^{t+r} \varphi(u) \varepsilon(t+r-u) d u-\int_{0}^{t} \varphi(u) \varepsilon(t-u) d u\right) \\
& =\int_{0}^{1} \frac{t}{r}[\varepsilon((t+r)(1-v)) \varphi(v(t+r))-\varepsilon(t(1-v)) \varphi(v t)] d v+\int_{0}^{1} \varepsilon((t+r)(1-v)) \varphi(v(t+r)) d v=: I_{r}^{(1)}+I_{r}^{(2)} .
\end{aligned}
$$


For every $r$ in $(0,1)$, one has $I_{r}^{(2)}=\int_{0}^{t+1} \mathbf{1}_{(0, t+r)}(u) \frac{\varepsilon(u)}{t+r} \varphi(t+r-u) d u$. Since $t$ and $\varepsilon$ are positive, Lebesgue's dominated convergence theorem applies and allows one to write that $\lim _{r \rightarrow 0} I_{r}^{(2)}=$ $\int_{0}^{t} \frac{\varepsilon(u)}{t} \varphi(t-u) d u$ and thus 12 that $\lim _{r \rightarrow 0} I_{r}^{(2)}=\frac{1}{t} \int_{0}^{t} \varphi(u) \varepsilon(t-u) d u$. Besides, $I_{r}^{(1)}$ can be written under the following form:

$I_{r}^{(1)}=\int_{0}^{1} t \varepsilon((t+r)(1-v))\left(\frac{\varphi(v(t+r))-\varphi(v t)}{r}\right) d v+\int_{0}^{1} \frac{t}{r} \varphi(v t)(\varepsilon((t+r)(1-v))-\varepsilon(t(1-v))) d v=: J_{r}^{(1)}+J_{r}^{(2)}$.

The exact same method as the one used to compute $\lim _{r \rightarrow 0} I_{r}^{(2)}$ applies and allows one to write:

$$
\lim _{r \rightarrow 0} J_{r}^{(1)}=\int_{0}^{1} t v \varphi^{\prime}(v t) \varepsilon(t(1-v)) d v=\frac{1}{t} \int_{0}^{t} u \varphi^{\prime}(u) \varepsilon(t-u) d u
$$

Having in mind that $\varepsilon^{2}(r)=\left(\gamma^{2}\right)^{\prime}(r)$, an integration by parts in $J_{r}^{(2)}$ yields:

$$
\begin{aligned}
J_{r}^{(2)}= & \varphi(0)\left(\frac{t}{t+r} \frac{(E(t+r)-E(t))}{r}-\frac{E(t)}{t+r}\right)-\frac{t}{t+r} \int_{0}^{1} \varphi^{\prime}(v t) E((t+r)(1-v)) d v \\
& +\underbrace{\frac{t}{r} \int_{0}^{1} \varphi^{\prime}(v t)(E((t+r)(1-v))-E(t(1-v))) d v}_{=: K_{r}} .
\end{aligned}
$$

It is then clear that:

$$
\lim _{r \rightarrow 0} J_{r}^{(2)}=\varphi(0)\left(\varepsilon(t)-\frac{E(t)}{t}\right)-\frac{1}{t} \int_{0}^{t} \varphi^{\prime}(u) E(t-u) d u+\lim _{r \rightarrow 0} K_{r} .
$$

Thus, it only remains to determine $\lim _{r \rightarrow 0} K_{r}$. An integration by parts in $K_{r}$ yields:

$K_{r}=\varphi^{\prime}(0)\left(\frac{t}{t+r} \frac{\mathscr{E}(t+r)-\mathscr{E}(t)}{r}-\frac{\mathscr{E}(t)}{t+r}\right)-\frac{t}{t+r} \int_{0}^{1} \varphi^{\prime \prime}(v t) \mathscr{E}(t(1-v)) d v+\frac{t^{2}}{t+r} \int_{0}^{1} \varphi^{\prime \prime}(v t)\left(\frac{\mathscr{E}((t+r)(1-v))-\mathscr{E}(t(1-v))}{r}\right) d v$.

One then gets, after a change of varaible:

$$
\lim _{r \rightarrow 0} K_{r}=\varphi^{\prime}(0)\left(E(t)-\frac{\mathscr{E}(t)}{t}\right)-\frac{1}{t} \int_{0}^{t} \varphi^{\prime \prime}(u) \mathscr{E}(t-u) d u+\int_{0}^{t} \varphi^{\prime \prime}(u)\left(1-\frac{u}{t}\right) E(t-u) d u .
$$

Finally, gathering $\lim _{r \rightarrow 0} I_{r}^{(2)}$ and Equalities (B.2) to (B.4) yields:

$$
\begin{aligned}
& \lim _{r \rightarrow 0} I_{r}=\frac{1}{t} \int_{0}^{t} \varphi(u) \varepsilon(t-u) d u+\frac{1}{t} \int_{0}^{t} \varphi^{\prime}(u)(u \varepsilon(t-u)-E(t-u)) d u \\
& \quad+\frac{1}{t} \int_{0}^{t} \varphi^{\prime \prime}(u)((t-u) E(t-u)-\mathscr{E}(t-u)) d u+\varphi(0)\left(\varepsilon(t)-\frac{E(t)}{t}\right)+\varphi^{\prime}(0)\left(E(t)-\frac{\mathscr{E}(t)}{t}\right) .
\end{aligned}
$$

This is nothing but (3.5). Let us now show that $t \mapsto\left|\Phi^{\prime}(t)\right|_{-q} \in \underset{b \in \mathbf{R}_{+}^{*}}{\bigcap} L^{2}((0, b)), \forall q \geq 3$. Let $b$ be a positive real and $q$ be an integer such that $q \geq 3$. It is sufficient to show that the map $t \mapsto\left|\Phi^{\prime}(t)\right|_{-q}^{2}$ belongs to $L^{1}((0, b))$. Using (3.5), one gets, for every integer $k \geq 2$,

$$
\left|<\Phi^{\prime}(t), e_{k}>\right| \leq \alpha_{k}\left(\varepsilon(t)+\frac{E(t)}{t}+M\right)
$$

where $\alpha_{k}:=\left\|e_{k}\right\|_{\infty}+\left\|e_{k}^{\prime}\right\|_{\infty}+\left\|e_{k}^{\prime \prime}\right\|_{\infty}$ and where $M:=(t+3) \sup _{s \in[0, t]} E(s)+\sup _{s \in[0, t]} \mathscr{E}(s)+\sup _{s \in(0, t]} \frac{\mathscr{E}(s)}{s}$. Using the relation $e_{k}^{\prime}(x)=\sqrt{\frac{k}{2}} e_{k-1}(x)-\sqrt{\frac{k+1}{2}} e_{k+1}(x)$ (see [Kuo96, p.354]) as well as Theorem 2.1] one easily obtains that $\alpha_{k} \leq 48(k+1)^{2} \sum_{l=-2}^{2}\left\|e_{k+l}\right\|_{\infty}^{2} \leq 250(k+1)^{2}$, for every integer $k \geq 2$. Having in mind the definition of $\mathcal{R}_{n}$ given in (3.4) it is then clear that there exists $C>0$ which does not depend on $q$ nor $t$ such that:

\footnotetext{
${ }^{12}$ Note that one could also have used [MV05, Remark 3] and assume that $\left(\gamma^{2}\right)^{\prime}$ (and hence $\varepsilon$ ) is non-increasing.
} 


$$
\left|\Phi^{\prime}(t)\right|_{-q}^{2}=\sum_{k=0}^{+\infty} \mid\left\langle\Phi^{\prime}(t), e_{k}>\left.\right|^{2}(2 k+2)^{-2 q} \leq \ell(t) \cdot \sum_{k=0}^{+\infty} \alpha_{k}^{2}(2 k+2)^{-2 q} \leq C \cdot \ell(t) \cdot \mathcal{R}_{2-q},\right.
$$

where we have set $\ell(t):=\left(\varepsilon(t)+\frac{E(t)}{t}+M\right)^{2}$. It then remains to show that both $\varepsilon^{2}$ and $t \mapsto \frac{E(t)^{2}}{t^{2}}$ belong to $L^{1}((0, b))$. The first part is clear since $\varepsilon^{2}=\left(\gamma^{2}\right)^{\prime}$. Moreover, in a neighborhood of 0 one has 13 $E(t) \leq 2 t \varepsilon(t)$, for $t \neq 0$. One therefore has: $\int_{0}^{b}\left(\frac{E(t)}{t}\right)^{2} d t \leq 4 \int_{0}^{b} \varepsilon^{2}(t) d t=4 \gamma^{2}(b)<+\infty$, which ends the proof.

\section{References}

[AMN01] E. Alòs, O. Mazet, and D. Nualart. Stochastic calculus with respect to Gaussian processes. Ann. Probab., 29(2):766-801, 2001.

[Bal03] V. Bally. An elementary introduction to Malliavin calculus. rapport de recherche no 4718, INRIA, 2003.

[BDM10] B. Boufoussi, M. Dozzi, and R. Marty. Local time and Tanaka formula for a Volterra-type multifractional Gaussian process. Bernoulli, 16(4):1294-1311, 2010.

[Ben03a] C. Bender. An Itô formula for generalized functionals of a fractional Brownian motion with arbitrary Hurst parameter. Stochastic Processes and their Applications, 104:81-106, 2003.

[Ben03b] C. Bender. An S-transform approach to integration with respect to a fractional Brownian motion. Bernoulli, $9(6): 955-983,2003$.

[BJR97] A. Benassi, S. Jaffard, and D. Roux. Elliptic Gaussian random processes. Rev. Mat. Iberoamericana, 13(1):1990, 1997.

[BSØW04] F. Biagini, A. Sulem, B. Øksendal, and N.N. Wallner. An introduction to white-noise theory and Malliavin calculus for fractional Brownian motion. Proc. Royal Society, special issue on stochastic analysis and applications, pages 347-372, 2004.

[CLV14] S. Corlay, J. Lebovits, and J. Lévy Véhel. Multifractional stochastic volatility models. Math. Finance, 24(2):364-402, 2014.

[CN05] P. Cheridito and D. Nualart. Stochastic integral of divergence type with respect to fractional Brownian motion with Hurst parameter $H \in\left(0, \frac{1}{2}\right)$. Ann. Inst. H. Poincaré Probab. Statist., 41(6):1049-1081, 2005.

[Cou07] L. Coutin. An Introduction to (Stochastic) Calculus with Respect to Fractional Brownian Motion. Séminaire de Probabilités XL, 1899:3-65, 2007.

[EVdH03] R.J. Elliott and J. Van der Hoek. A general fractional white noise theory and applications to finance. Mathematical Finance, 13(2):301-330, 2003.

[Föl81] H. Föllmer. Calcul d'Itô sans probabilités. In Seminar on Probability, XV (Univ. Strasbourg, Strasbourg, 1979/1980) (French), volume 850 of Lecture Notes in Math., pages 143-150. Springer, Berlin, 1981.

[FV10] P.K. Friz and N.B. Victoir. Multidimensional Stochastic Processes as Rough Paths: Theory and Applications. Cambridge University Press, 2010.

[GSV07] D. Gasbarra, T. Sottinen, and E. Valkeila. Gaussian bridges. In Stochastic analysis and applications, volume 2 of Abel Symp., pages 361-382. Springer, Berlin, 2007.

[GT82] B. Gaveau and P. Trauber. L'intégrale stochastique comme opérateur de divergence dans l'espace fonctionnel. J. Funct. Anal., 46(2):230-238, 1982.

[HH93] T. Hida and M. Hitsuda. Gaussian processes, volume 120 of Translations of Mathematical Monographs. American Mathematical Society, Providence, RI, 1993. Translated from the 1976 Japanese original by the authors.

[Hid75] T. Hida. Analysis of Brownian functionals. Carleton Univ., Ottawa, Ont., 1975. Carleton Mathematical Lecture Notes, No. 13.

[Hit72] M. Hitsuda. Formula for Brownian partial derivatives, 1972. Second Japan-USSR Symposium on Probability Theory, Kyoto, 111-114.

[Hit78] M. Hitsuda. Formula for Brownian partial derivatives, 1978. Proceedings of Faculty of Integrated Arts and Sciences. Hiroshima University III-4, 1-15.

\footnotetext{
${ }^{13}$ See [MV05, p.396] for a proof of this fact.
} 
[HKPS93] T. Hida, H. Kuo, J. Potthoff, and L. Streit. White Noise. An Infinite Dimensional Calculus, volume 253. Kluwer academic publishers, 1993.

[HOUZ10] H. Holden, B. Oksendal, J. Ubøe, and T. Zhang. Stochastic Partial Differential Equations, A Modeling, White Noise Functional Approach. Springer, second edition, 2010.

[HP57] E. Hille and R.S. Phillips. Functional Analysis and Semi-Groups, volume 31. American Mathematical Society, 1957.

[HS04] T. Hida and Si Si. An innovation approach to random fields. World Scientific Publishing Co. Inc., River Edge, NJ, 2004. Application of white noise theory.

[HV03] C. Houdré and J. Villa. An example of infinite dimensional quasi-helix. In Stochastic models (Mexico City, 2002), volume 336 of Contemp. Math., pages 195-201. Amer. Math. Soc., Providence, RI, 2003.

[Jan97] S. Janson. Gaussian Hilbert Spaces, volume 129 of Cambridge Tracts in Mathematics. Cambridge University Press, Cambridge, 1997.

[Kol40] A. Kolmogorov. Wienersche Spiralen und einige andere interessante Kurven in Hilbertsche Raum. C. R. (Dokl.) Acad. Sci. URSS, 26:115-118, 1940.

[KR10] I. Kruk and F. Russo. Malliavin Skorohod calculus and Paley-Wiener integral for covariance singular processes. Preprint, 2010.

[KRT07] I. Kruk, F. Russo, and C. A. Tudor. Wiener integrals, Malliavin calculus and covariance measure structure. J. Funct. Anal., 249(1):92-142, 2007.

[Kub83] Izumi Kubo. Itô formula for generalized Brownian functionals. In Theory and application of random fields (Bangalore, 1982), volume 49 of Lect. Notes Control Inf. Sci., pages 156-166. Springer, Berlin, 1983.

[Kuo96] H.H. Kuo. White Noise Distribution Theory. CRC-Press, 1996.

[Leb13] J. Lebovits. From stochastic integral w.r.t. fractional Brownian motion to stochastic integral w.r.t. multifractional Brownian motion. Ann. Univ. Buchar. Math. Ser., 4(LXII)(1):397-413, 2013.

[Leb17] Joachim Lebovits. Local Times of Gaussian Processes: Stochastic Calculus wrt Gaussian Processes Part II. Preprint, 2017.

[LLV14] J. Lebovits and J. Lévy Véhel. White noise-based stochastic calculus with respect to multifractional Brownian motion. Stochastics An International Journal of Probability and Stochastic Processes, 86(1):87-124, 2014.

[LLVH14] J. Lebovits, J. Lévy Véhel, and E. Herbin. Stochastic integration with respect to multifractional Brownian motion via tangent fractional Brownian motions. Stochastic Process. Appl., 124(1):678-708, 2014.

[LN05] J. A. León and D. Nualart. An extension of the divergence operator for Gaussian processes. Stochastic Process. Appl., 115(3):481-492, 2005.

[LN12] P. Lei and D. Nualart. Stochastic calculus for Gaussian processes and application to hitting times. Commun. Stoch. Anal., 6(3):379-402, 2012.

[Mis08] Y.S. Mishura. Stochastic calculus for fractional Brownian motion and related processes, volume 1929 of Lecture Notes in Mathematics. Springer-Verlag, Berlin, 2008.

[MV05] O. Mocioalca and F. Viens. Skorohod integration and stochastic calculus beyond the fractional Brownian scale. J. Funct. Anal., 222(2):385-434, 2005.

[MVN68] B. Mandelbrot and J.W. Van Ness. Fractional Brownian motions, fractional noises and applications. SIAM Rev., 10:422-437, 1968.

[MY06] R. Mansuy and M. Yor. Random times and enlargements of filtrations in a Brownian setting. Springer-Verlag New York, Inc., 2006.

[NT06] D. Nualart and M.S. Taqqu. Wick-Itô formula for Gaussian processes. Stoch. Anal. Appl., 24(3):599-614, 2006.

[Nua05] D. Nualart. A white noise approach to fractional Brownian motion. In Stochastic analysis: classical and quantum, pages 112-126. World Sci. Publ., Hackensack, NJ, 2005.

[Nua06] D. Nualart. The Malliavin Calculus and Related Topics. Springer, 2006.

[PLV95] R. Peltier and J. Lévy Véhel. Multifractional Brownian motion: definition and preliminary results, 1995. rapport de recherche de l'INRIA, $n^{0} 2645$. 
[RV07] F. Russo and P. Vallois. Elements of stochastic calculus via regularization. In Séminaire de Probabilités XL, volume 1899 of Lecture Notes in Math., pages 147-185. Springer, Berlin, 2007.

[RY99] D. Revuz and M. Yor. Continuous martingales and Brownian motion, volume 293 of Grundlehren der Mathematischen Wissenschaften [Fundamental Principles of Mathematical Sciences]. Springer-Verlag, Berlin, third edition, 1999.

[Si12] Si Si. Introduction to Hida distributions. World Scientific Publishing Co. Pte. Ltd., Hackensack, NJ, 2012.

[Sko75] A. V. Skorohod. On a generalization of the stochastic integral. Teor. Verojatnost. i Primenen., 20(2):223-238, 1975.

[Str83] C. Stricker. Semimartingales gaussiennes-application au problème de l'innovation. Z. Wahrsch. Verw. Gebiete, 64(3):303-312, 1983.

[SV13] T. Sottinen and L. Viitassaari. Pathwise integrals and Itô-Tanaka formula for Gaussian processes. Preprint, 2013. Preprint, arXiv:1307.3578

[SV14] T. Sottinen and L.. Viitasaari. Stochastic Analysis of Gaussian Processes via Fredholm Representation. 2014. Preprint, arXiv:1410.2230.

[Tha93] S. Thangavelu. Lectures of Hermite and Laguerre expansions. Princeton University Press, 1993.

[Wid44] D.V. Widder. Positive temperatures on an infinite rod. Trans. Amer. Math. Soc., 55:85-95, 1944.

[You36] L. C. Young. An inequality of the Hölder type, connected with Stieltjes integration. Acta Math., 67(1):251-282, 1936.

[Zä98] M. Zähle. Integration with respect to fractal functions and stochastic calculus. I. Probab. Theory Related Fields, 111(3):333-374, 1998 